$$
\begin{gathered}
\text { UNIVERSIDADE DE SÃO PAULO } \\
\text { INSTITUTO DE FÍSICA DE SÃO CARLOS } \\
\text { DEPARTAMENTO DE FíSICA E INFORMÁTICA }
\end{gathered}
$$

\title{
GRUPO DE RENORMALIZAÇÃO NUMÉRICO COM BANDA ASSIMÉTRICA
}

Amilton de Matos Teixeira

\begin{abstract}
Tese apresentada ao Instituto de Física de São Carlos,
Universidade de São Paulo, para obtenção do Título de Doutor em Física.
\end{abstract}

Orientador: Prof. Dr. Valter Luiz Líbero 
AUTORIZO A REPRODUÇÃO E DIVULGAÇÃO TOTAL OU PARCIAL DESTE TRABALHO, POR QUALQUER MEIO CONVENCIONAL OU ELETRÔNICO, PARA FINS DE ESTUDO E PESQUISA, DESDE QUE CITADA A FONTE.

Ficha catalográfica elaborada pelo Serviço de Biblioteca e Informação IFSC/USP

Teixeira, Amilton de Matos.

Grupo de renormalização numérico com banda assimétrica / Amilton de Matos Teixeira; orientador Valter Luiz Líbero.-- São Carlos, 2007. $98 \mathrm{p.}$

Tese (Doutorado em Física - Área de concentração: Física Básica ) - Instituto de Física de São Carlos da Universidade de São Paulo.

1. Grupo de renormalização numérico. 2. Assimetria. 3. Campo magnético. I. Grupo de renormalização numérico com banda assimétrica. 
MEMBROS DA COMISSÃO JULGADORA DA TESE DE DOUTORADO DE AMILTON DE MATOS TEIXEIRA APRESENTADA AO INSTITUTO DE FISICA DE SÃO CARLOS, UNIVERSIDADE DE SÃO PAULO, EM 12/07/2007.

COMISSÃO JULGADORA:

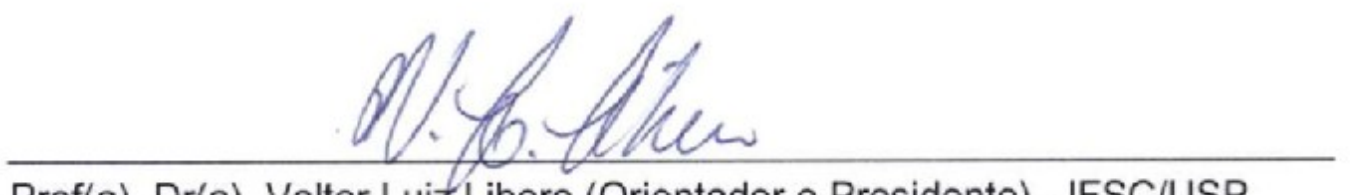

Prof(a). Dr(a). Valter Luiz Libero (Orientador e Presidente) - IFSC/USP

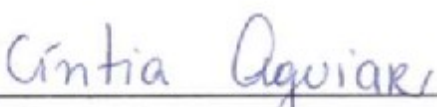

Prof(a). Dr(a). Cintia Aguiar - UDESC

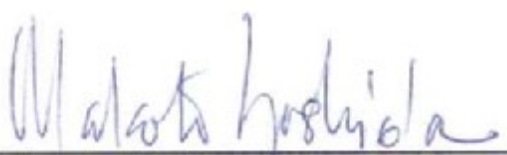

Prof(a). Dr(a). Makoto Yoshida - UNESP / Rio Claro

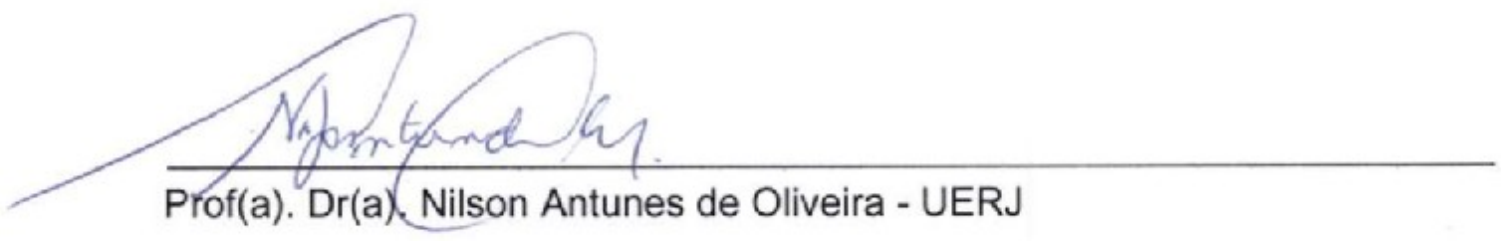

Prof(a). Dr(a) Nilson Antunes de Oliveira - UERJ

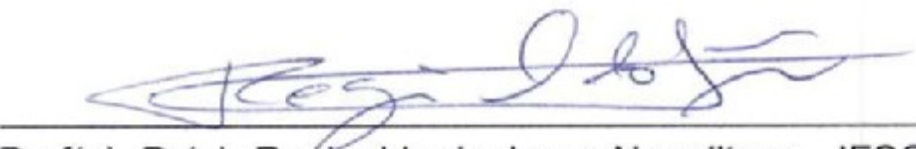

Prof(a). Dr(a). Reginaldo de Jesus Napolitano - IFSC/USP 


\section{Agradecimentos}

- ao professor Valter, exímio docente e paciente orientador;

- a todos os funcionários do IFSC, em especial a Cris cuja dedicação transcendeu as atribuições de secretária tendo sido co-responsável por este trabalho ter sido concluído;

- aos meus amigos e colegas, particularmente aqueles com os quais sempre pude contar:

Fabiano, Mariana, Paulo Franzen, Viviane, Vivaldo;

- aos professores do grupo pela convivência enriquecedora;

- aos meus pais João e Iraci por amarem incondicionalmente seus filhos;

- a Érika que, desde o primeiro relatório da FAPESP, divide comigo as ansiedades, os sonhos e as expectativas de uma vida cada vez mais plena;

- à FAPESP que apoiou este projeto e me sustentou durante quatro anos;

- a Deus.

"DA FELICIDADE

Quantas vezes a gente, em busca da ventura,

Procede tal e qual o avozinho infeliz:

Em vão, por toda parte, os óculos procura

Tendo-os na ponta do nariz!"

Mário Quintana 


\section{RESUMO}

Este trabalho apresenta um tratamento do Grupo de Renormalização Numérico (GRN) que permite a inclusão de assimetrias na banda de condução, como a provocada por campo magnetostático aplicado a sistemas de spins localizados em metais. Resultados para calor específico e suscetibilidade magnética de spin são obtidos para os modelos de Kondo de dois canais (MK2C), de Falikov-Kimball (MFK) e o modelo de nível ressonante na presença dessas assimetrias. São apresentadas soluções para lidar com as oscilações espúrias das curvas do calor específico, inerentes ao GRN. Tais abordagens, contribuíram de forma determinante para a viabilidade dos resultados apresentados aqui para essas grandezas. 


\section{ABSTRACT}

This work presents an approach to the Numerical Renormalization Group which allows for asymmetries in the conduction band, as those brought about by magnetostatic field applied to a system of localized spin in metal. Results for specific heat and magnetic susceptibility are shown for the two-channel Kondo model, as well for the Falikov-Kimball and resonant level models, in the presence of such asymmetry. In addition, novel treatments were described in order to smooth out the curves of specific heat from the oscilating profile that comes along from NRG calculations. This was of outmost importance for the viability of the results presented here. 


\section{Sumário}

1 Introdução 5

1.1 Motivação . . . . . . . . . . . . . . . . . . . . . . . . . . . 5

1.2 Alguns Desenvolvimentos Históricos Pertinentes . . . . . . . . . . . . . . . . . . 6

1.3 Resultados Principais Deste Trabalho . . . . . . . . . . . . . . . . . . . . 8

2 Grupo de Renormalização com banda de condução assimétrica 10

2.1 Discretização Logarítmica da banda . . . . . . . . . . . . . . . . . . . . . 10

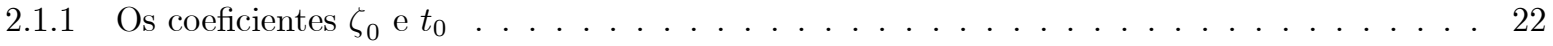

2.1.2 Justificando Truncamento para $N$ finito . . . . . . . . . . . . . . . . . . . 23

2.1.3 Por que discretizar banda + campo juntos . . . . . . . . . . . . . . . . . . . 24

2.1.4 Recuperando as bandas com $\operatorname{spin} \mu= \pm \ldots . . . . . . . . . . . . . . . .24$

3 Modelo de Nível Ressonante 26

3.1 Expressões analíticas para o MNR . . . . . . . . . . . . . . . . . . . . . 30

3.2 Resultados numéricos para o MNR . . . . . . . . . . . . . . . . . . . . . . . . 32

4 Modelo de Falicov-Kimball (MFK) 38

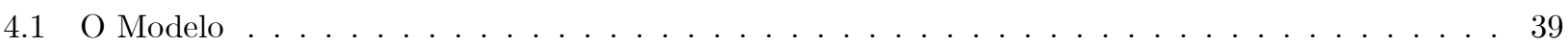

4.2 Grupo de Renormalização Numérico . . . . . . . . . . . . . . . . . . . . . . . . . . 40

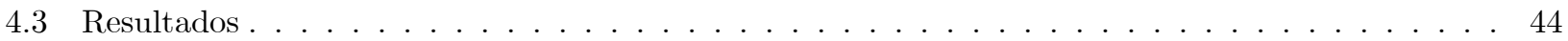

$4.3 .1 \mathbf{G}=\mathbf{V}=\mathbf{0} \ldots \ldots \ldots \ldots \ldots \ldots$

$4.3 .2 \mathbf{G} \neq \mathbf{0}$ e $\mathbf{V}=\mathbf{0} \ldots \ldots \ldots \ldots \ldots$

$4.3 .3 \mathbf{G}=\mathbf{0}, \mathbf{V} \neq \mathbf{0} \ldots \ldots \ldots \ldots \ldots \ldots$

$4.3 .4 \quad G \neq 0$ e $V \neq 0 \ldots \ldots \ldots \ldots \ldots \ldots \ldots$

4.4 Possíveis Generalizações . . . . . . . . . . . . . . . . . . . . . . . . . . . . 51

5 Modelo de Kondo de Dois Canais 54

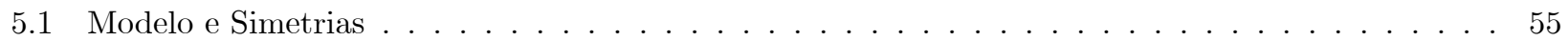


5.2 A transformação do Grupo de Renormalização em duas fases . . . . . . . . . . . . . . . . . 58

5.3 Diagonalização Iterativa $\ldots \ldots \ldots \ldots \ldots \ldots$

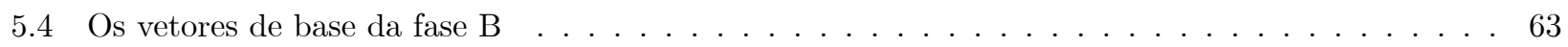

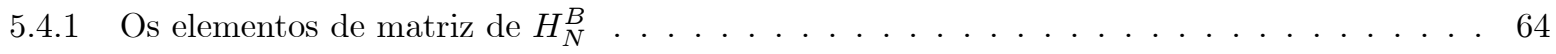

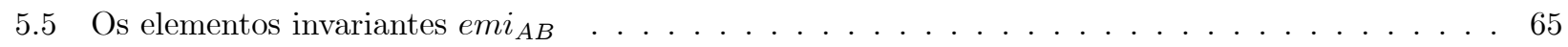

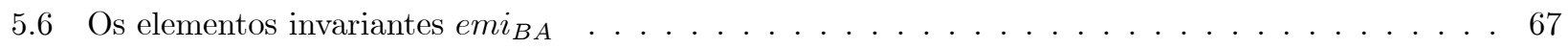

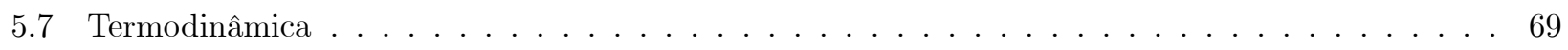

5.7 .1 Método da intersecção . . . . . . . . . . . . . . . . . . . 70

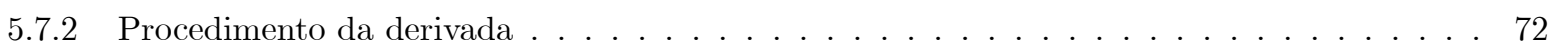

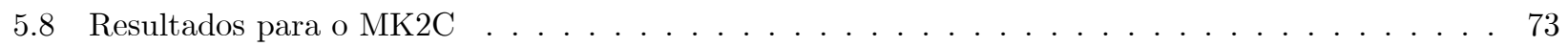

6 Conclusões $\quad 77$

7 Apêndice A - Função Poligama $r$

8 Apêndice B - Quantidades termodinâmicas para a banda livre $\quad 81$

9 Apêndice C - Os operadores $f_{n, \sigma, \alpha}$ são tensores $\quad 84$

10 Apêndice D - Bloco Diagonalização de $H_{0, B} \quad 86$

\section{Lista de Figuras}

1 Pablo et. al. [1] montaram um sistema que apresenta efeito Kondo convencional, orbital e SU(4). Consiste num nanotubo de carbono crescido sobre um substrato silício com uma camada de dióxido de silício. Uma tensão V é aplicada através de contatos de ouro sendo a corrente I medida para um dado valor de tensão de gate $V_{G}$. Ajustando-se a tensão de gate, pode-se controlar a número de elétrons no poço quântico criado pelo nanotubo de tal forma que nele fique apenas um elétron em regime de Coulomb blockade simulando assim uma impureza magnética. . $\quad 6$ 
2 A figura da esquerda ilustra o aumento de energia da sub-banda $\mu=+$ devido à aplicação do campo magnético $B$. A figura da direita, por sua vez, representa a diminuição da energia da sub-banda $\mu=-$ devido ao mesmo campo. $\ldots \ldots \ldots \ldots \ldots \ldots \ldots \ldots$

3 Aqui, as duas sub-bandas de spins opostos tem seus limites de energia redefinidos de tal forma que a energia de Fermi, $\epsilon_{F}$, da banda completa seja igual a zero. $\ldots \ldots \ldots \ldots \ldots$

4 Discretização logarítmica da banda de condução segundo preceitua o GRN. Porém, foi introduzido o parâmetro de discretização $z$, além do campo magnético aplicado $B \ldots \ldots \ldots \ldots$. . . . . 13

5 Diagrama de níveis do MNR. Os níveis unicamente ocupados apresentam largura devido à hibridização com a banda de condução. O campo magnético quebra a degenescência destes níveis. . 27

6 Curvas de calor específico para o MNR para três valores de campo, comparando-se o resultado

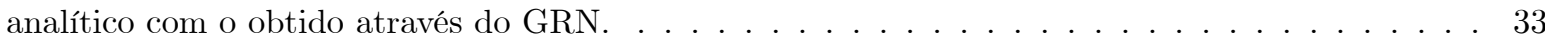

7 Curvas da entropia exponenciada, ou seja, do número de graus de liberdade correspondentes às curvas de calor específico da Fig. $(3.2) \ldots \ldots \ldots \ldots \ldots \ldots \ldots \ldots \ldots$

8 Curvas de calor específico para o caso em que a hibridização é dependente do spin. . . . . . . . . 35

9 Curvas da entropia exponenciada, ou seja, dos graus de liberdade correspondentes às curvas de calor específico da Fig. $(3.4) . \ldots \ldots \ldots \ldots \ldots \ldots \ldots \ldots \ldots$

10 Curvas para suscetibilidade de carga e calor específico do MFK num caso em que há solução analítica. A temperatura de pico do calor específico (curva azul) $T_{p i c o}$ é dada por $k_{B} T_{p i c o}=$ $0,416778 E_{d}^{*}$, onde $E_{d}^{*}=\left|E_{d}-B\right| \ldots \ldots \ldots \ldots \ldots \ldots \ldots \ldots \ldots \ldots$

11 Curvas de calor específico do MFK para diversos valores de $B$ para $G \neq 0$ e $V=0 \ldots \ldots$. . . . 47

12 Decrição gráfica da variação da posição do pico das curvas do calor específico do MFK em função do campo $B$, sendo $V=0$ e $E_{d}=0,001 D$, para dois valores do termo de espalhamento Coulombiano: $G=0,01 D$ e $G=0,02 D$. Notemos que os valores de $B$ a partir dos quais as inclinações das retas mudam de sinal é ligeiramente maior que os $G$ 's correspondentes. . . . . . . 48

13 Curvas da suscetibilidade de carga do MFK para diversos valores de $B$ para $V=0, G=0,01 D$

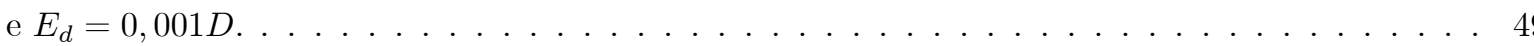


14 Curvas do calor específico do MFK para diversos valores de $B$, sendo $V=0,02 D, G=0$ e

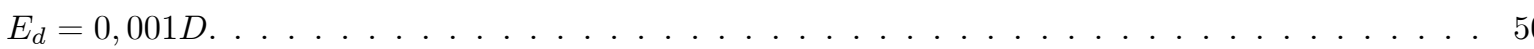

15 Curvas da suscetibilidade de carga do MFK para diversos valores de $B$, sendo $V=0,02 D, G=0$

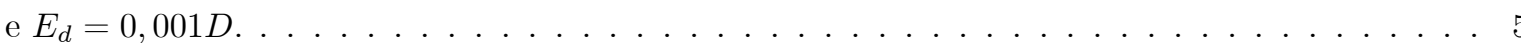

16 Curvas do calor específico (em vermelho) e da susceptibilidade (em azul) num intervalo em baixa temperatura evidenciando o comportamento linear de ambas grandezas para o caso em que $G=0$, sendo $E_{d}=0,001 D$ e $V=0,02 D$.

17 Curvas do calor específico para o calor específico do MFK para três valores de campo, sendo $G$, $V$, e $E_{d}$ simultaneamente diferentes de zero.

18 Curvas da suscetibilidade de carga para o calor específico do MFK para três intensidades de campo, para valores de $G, V$, e $E_{d}$ simultaneamente diferentes de zero. . . . . . . . . . 54

19 Comparação entre a média de duas curvas de calor específico com dois z's diferindo de 0.5 e a curva universal mostrando que os pontos de intersecção pertencem à curva desejada.

20 Interpolação linear utilizada no método da intersecção. Os pontos são resultados do GRN, enquanto $X_{i}$ e $Y_{i}$ são as coordenadas do ponto de intersecção. Os pontos azuis são para um determinado $z$, enquanto os vermelhos são para outro $z$ diferindo de 0,5 em relação ao dos pontos azuis.

21 Curvas da exponencial da entropia (graus de liberdade) evidenciando situação de "quase-simetria" de canais. $\mathrm{O}$ valor de $T_{K}$ é o correspondente a $J=0.08 D \ldots \ldots \ldots \ldots \ldots \ldots$

22 Curva para o calor específico para o MK2C mostrando casos em que os valores dos acopalhamentos

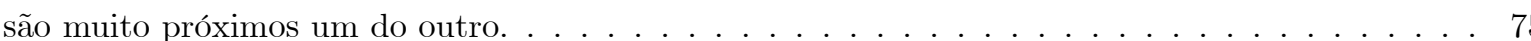

23 Curvas da suscetibilidade magnética para casos em que os valores dos acoplamentos são próximos

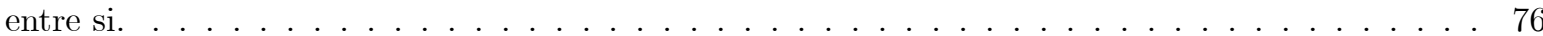




\section{Capítulo 1}

\section{Introdução}

\subsection{Motivação}

Fenômenos envolvendo materiais magnéticos são conhecidos desde a Grécia antiga, passando pela invenção da bússula pelos chineses por volta do ano 1000 e chegando até ao desenvolvimento tecnológico atual. Entretanto, do ponto de vista teórico, seu correto entendimento não foi possível antes do advento da Mecânica Quântica ao longo da primeira metade do século XX. Apesar do ferramental teórico já estar disponível, a descrição de materiais magnéticos e até mesmo a de sistemas com impurezas magnéticas é tarefa extremamente difícil, senão impossível em muitos casos. O que se faz então é lançar mão de modelos que capturam a essência do sistema real resguardando suas propriedades importantes, mas que ainda sejam tratáveis teoricamente.

Se por um lado modelos procuram representar sistemas físicos cada vez mais realisticamente, ou seja, o modelo se adequando ao sistema físico, curiosamente, o oposto também ocorre: dispositivos adequando-se a modelos. Encontramos muitos exemplos assim em sistemas semicondutores de pontos e poços quânticos, como aqueles à base de nanotubos de carbono exibindo efeitos antes só observáveis em sistemas-padrão de impurezas magnéticas em metais não magnéticos [1]. 


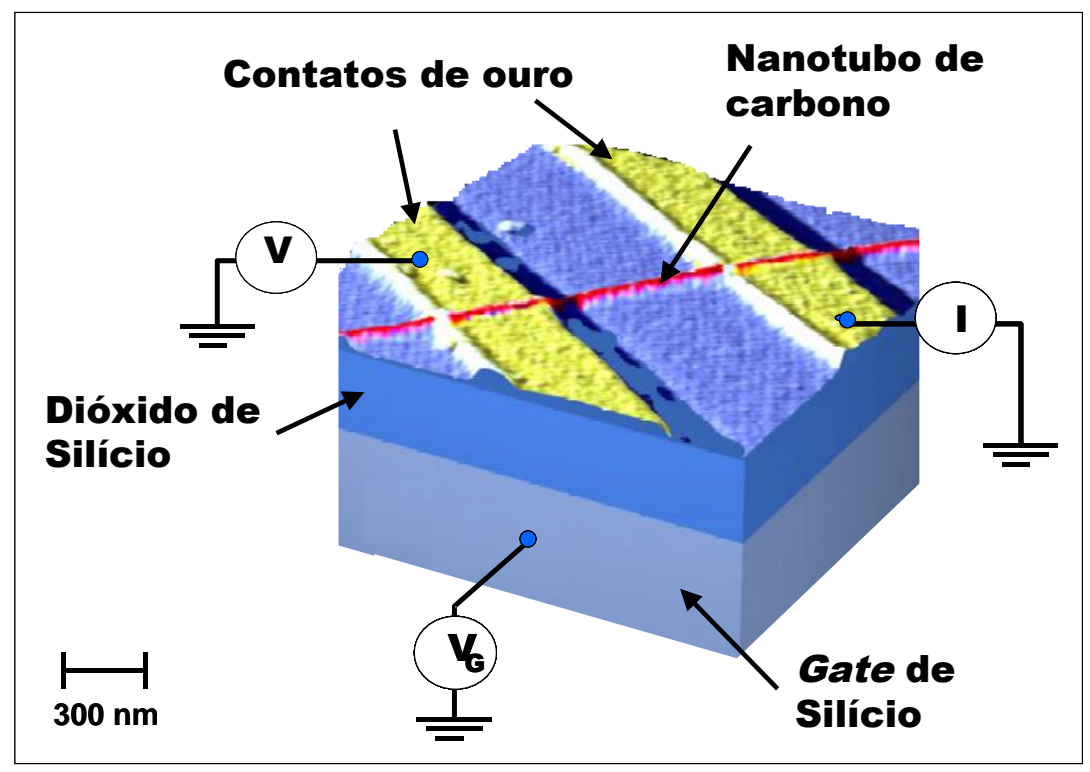

Figura 1.1: Pablo et. al. [1] montaram um sistema que apresenta efeito Kondo convencional, orbital e SU(4). Consiste num nanotubo de carbono crescido sobre um substrato silício com uma camada de dióxido de silício. Uma tensão $\mathrm{V}$ é aplicada através de contatos de ouro sendo a corrente I medida para um dado valor de tensão de gate $V_{G}$. Ajustando-se a tensão de gate, pode-se controlar a número de elétrons no poço quântico criado pelo nanotubo de tal forma que nele fique apenas um elétron em regime de Coulomb blockade simulando assim uma impureza magnética.

Apesar de não nos ocuparmos neste trabalho de suas implicações tecnológicas, o estudo de modelos de impurezas localizadas em meio metálico é de extrema utilidade prática. Como vimos antes, realizações de modelos de controle de spin tem sido imaginados e desenvolvidos para que tenhamos um dia dispositivos comercialmente viáveis fazendo da spintrônica uma realidade também fora dos institutos de pesquisa.

Do ponto de vista acadêmico, torna-se necessário criar técnicas que possam abordar modelos cada vez mais realistas. O uso do Grupo de Renormalização Numérico (GRN) [2] adaptado, por exemplo, para inclusão de campo magnético, ou de banda de condução assimétrica, ou ainda com banda de condução com vários graus de liberdade (canais) torna sua implementação mais realista. Com isso, a determinação de propriedades termodinâmicas como entropia, calor específico ou suscetibilidade magnética, cuja obtenção por si só não é trivial, terá vantagens a serem usufruídas em trabalhos 
posteriores quando comparações com sistemas mais realistas forem necessárias. Em suma, o que norteia este trabalho de tese é dar um passo além das implementações usuais do GRN para sistemas de impurezas localizadas em um mar de elétrons.

\subsubsection{Alguns Desenvolvimentos Históricos Pertinentes}

Tendo em mente o mecanismo de espalhamento por fônons, é de se esperar que a resistividade dos metais diminua quando esfriados. De fato, isso é o que é geralmente observado. Contudo, em 1930, Meissner e Voigt depararam-se com a inusitada situação na qual a resistividade do ouro aumentava à medida em que a temperatura diminuía abaixo dos $10 \mathrm{~K}$ chegando a um valor finito para $T \rightarrow 0$ $K$ [3]. As teorias existentes não davam conta desse fenômeno. Experimentalmente, verificou-se que a temperatura para a qual ocorria o mínimo era proporcional a $1 / c^{5}$, sendo $c$ a concentração de impurezas magnéticas (por exemplo, ferro) presentes no metal hospedeiro não magnético (por exemplo, ouro). Também observou-se que a profundidade do mínimo em relação à resistividade a $\mathrm{T}=0 \mathrm{~K}$ era proporcional a $c$.

Jun Kondo mostrou que esse efeito era devido exclusivamente à presença de impurezas magnéticas acoplando-se com a banda de condução e que o acoplamento entre elas (as impurezas) não era relevante (ao menos em baixas concentrações) [4]. Propôs, então, o termo de interação $J \vec{s} \cdot \vec{S}_{I}$, onde $\vec{S}_{I}$ é um spin localizado (de uma impureza em geral) e $\vec{s}$ o spin da banda. Em 1964, utilizando um cálculo perturbativo, obteve a condutividade elétrica desse modelo, que exibia um mínimo numa certa temperatura, e também explicava a dependência com a concentração das impurezas magnéticas. Contudo, seu cálculo perturbativo introduzia uma divergência logarítmica na resistividade para temperaturas abaixo da hoje denominada temperatura Kondo. Isso exigia um tratamento mais rigoroso, que foi feito por K. G. Wilson com o Grupo de Renormalização Numérico em 1975 [5].

Wilson elaborou então o Grupo de Renormalização Numérico (GRN) e aplicou aos modelos 
de impureza magnética localizada em meio metálico, como os modelos de Kondo e Anderson [4, 5, 6]. A abordagem de Wilson é não perturbativa e com isso não apresenta a indesejável divergência logarítmica advinda de cálculos perturbativos. Esse novo procedimenteo consiste, como veremos adiante em detalhe, na discretização logarítmica da banda de condução e a definição de uma nova base para o Hamiltoniano de Kondo feita de estados ao redor da impureza.

Nesses modelos a impureza contém elétrons localizados, tipicamente elétrons de orbital $f$, ou mesmo $d$, mas a degenerescência destes é completamente desconsiderada. Isto pode ser alcançado, em muitos casos, por um campo cristalino altamente assimétrico que levantaria a degenerescência desses orbitais. Dando um passo além desta aproximação, Noziéres e Bladin [7] atribuíram novos números quânticos, conhecidos como canais, aos elétrons da banda de condução; esses canais seriam o resultado da ação de impurezas, com estrutura complexa, sobre os elétrons de condução. Desta forma, incorpora-se efetivamente aquela degerescência orbital.

\subsection{Resultados Principais Deste Trabalho}

Para tornar um problema de muitos corpos tratável numericamente, no GRN discretiza-se a banda de condução. Isto significa que qualquer energia $\varepsilon$ de excitação partícula-buraco da banda será considerada dentro do intervalo $\Lambda^{-m-1} \leq|\varepsilon| \leq \Lambda^{-m}$, para dados $\Lambda>1$ e $m>0$, sendo este último um número inteiro (ver Fig. (2.3) no Cap. 2). Os resultados da termodinâmica através do GRN serão tão mais precisos quanto mais o parâmetro se aproxima de $\Lambda=1$, pois assim melhor se representa o contínuo da banda. Contudo, há um compromisso com o tempo de processamento do programa que aumenta consideravelmente se $\Lambda \approx 1$, limite ideal para se recuperar o contínuo da banda de condução. Na prática, trabalha-se com $\Lambda$ tipicamente no intervalo de 4 a 10 . Para valores assim, as curvas das propriedades termodinâmicas, quando exibidas em função da temperatura, apresentam oscilações espúrias, como mostramos adiante. Yoshida et. al. [8] adicionaram o parâmetro de discretização $z$ de 
tal forma que agora $\varepsilon$ está no intervalo $\Lambda^{-m-z-1} \leq|\varepsilon| \leq \Lambda^{-m-z}$. A média das curvas obtidas para diferentes valores deste parâmetro elimina aquelas sinuosidades advindas da discretização.

Este trabalho contribui para expandir a aplicação do GRN em duas direções:

1. generaliza a discretização usual da banda de condução a dois parâmetros $(\Lambda$ e $z$ ) para bandas assimétricas em relação ao nível de Fermi, tal qual será quando tivermos campo magnético externo agindo no sistema, ou bandas longe do semipreenchimento. Desenvolvemos o procedimento analítico bem como subrotinas (em Maple) que fornecem os elementos de matriz necessários à discretização assimétrica. Aplicamos inicialmente a discretização assimétrica ao modelo de Nível Ressonante computando calor específico, entropia e suscetibilidade magnética em função da temperatura; essas funções são comparadas com as já conhecidas na literatura. Em seguida aplicamos ao modelo de Falicov-Kimball [9] onde fizemos, pela primeira vez, um estudo da dependência do calor específico com a assimetria da banda e da suscetibilidade de carga para com um campo externo aplicado às cargas do sistema;

2. apresenta procedimentos alternativos para se recuperar o contínuo da banda de condução e se livrar das oscilações espúrias decorrentes da discretização da banda - particularmente para o calor específico que apresenta oscilações de amplitudes enormes. Aqui voltamos ao caso de bandas simétricas em torno do nível de Fermi, mas levamos em conta dois graus de liberdade eletrônicos na banda, isto é, consideramos o modelo de Kondo de dois canais e com o GRN implementado em duas fases - essa implementação em duas fases já fora feita para modelo de Anderson de duas impurezas e um canal num cálculo da densidade espectral [10].

Uma das intenções iniciais deste trabalho era estudar o Modelo de Kondo de Dois Canais na presença de campo magnético. Almejamos que os desenvolvimentos aqui apresentados preparem terreno para futuro trabalho nessa linha. 


\section{Capítulo 2}

\section{Grupo de Renormalização com banda}

\section{de condução assimétrica}

Neste Capítulo descrevemos em detalhes como o Grupo de Renormalização Numérico (GRN) é implementado para bandas de condução assimétricas. Isso será aplicado, nos próximos Capítulos, aos Modelos de Nível Ressonante e de Falicov-Kimball.

\subsection{Discretização Logarítmica da banda}

Primeiramente, consideremos uma banda de condução que se estende da energia $\epsilon=0$ a $\epsilon=2 D$, estando o nível de Fermi em $\epsilon_{F}=D$, portanto semipreenchida ou simétrica. Não é difícil nos convencermos de que, quando estamos falando de baixas excitações, por menor que sejam, sempre haverá uma ao redor do nível de Fermi. É o que se chama invariância de escala e gera as divergências logarítmicas em baixas temperaturas quando a abordagem é perturbativa. A discretização logarítmica da banda de condução proposta por Wilson [2] foi elaborada de forma a reproduzir essa invariância de escala em torno do nível de Fermi. Seguimos a idéia de Wilson, mas para uma banda assimétrica. Devido ao nosso interesse em estudar a influência de um campo magnetostático nas pro- 


\section{D}
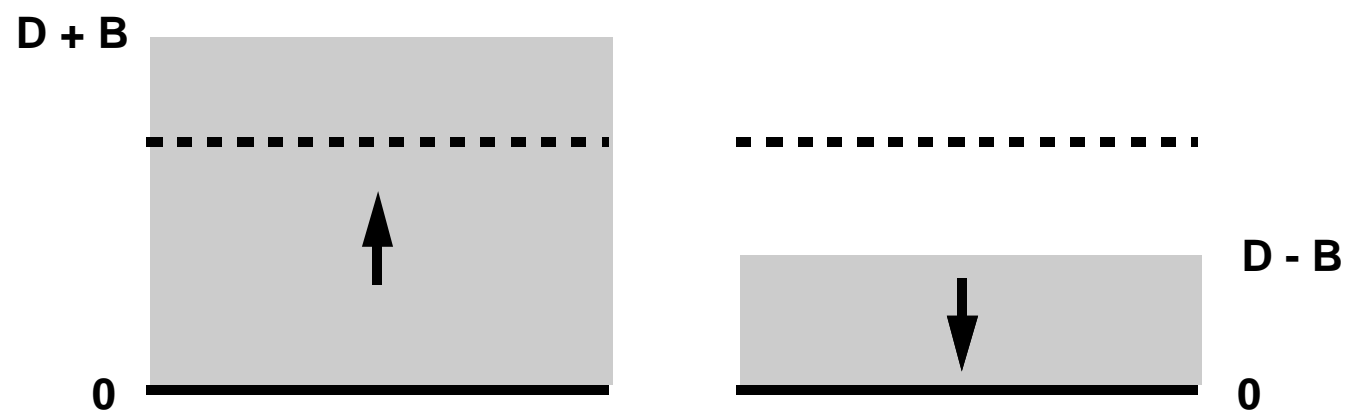

Figura 2.1: A figura da esquerda ilustra o aumento de energia da sub-banda $\mu=+$ devido à aplicação do campo magnético $B$. A figura da direita, por sua vez, representa a diminuição da energia da sub-banda $\mu=-$ devido ao mesmo campo.

priedades termodinâmicas tivemos que alterar o procedimento padrão do GRN. Isso é necessário porque o termo do Hamiltoniano devido à banda de condução,

$$
H_{B C}=\sum_{k, \mu} \epsilon_{\vec{k}} c_{\vec{k}, \mu}^{\dagger} c_{\vec{k}, \mu}
$$

deve conter a influência do campo da seguinte forma:

$$
H_{B C}=\sum_{\vec{k}, \mu} \epsilon \vec{k}_{\vec{k}, \mu}^{\dagger} c_{\vec{k}, \mu} c_{\vec{k}, \mu}-B \sum_{\vec{k}}\left(c_{\vec{k} \uparrow}^{\dagger} c_{\vec{k}, \uparrow}-c_{\vec{k} \downarrow}^{\dagger} c_{\vec{k}, \downarrow}\right)=\sum_{\vec{k}, \mu}(\epsilon \vec{k}-\mu B) c_{\vec{k}, \mu}^{\dagger} c_{\vec{k}, \mu},
$$

onde $B \equiv \hbar g \mu_{B} B_{z} / 2$, com $B_{z}$ a componente $z$ do campo magnético aplicado e $\mu= \pm$.

Podemos dizer que temos duas bandas de condução: uma com componente de spin $\mu=+$ e outra com $\mu=-$. Os estados com $\mu=+$ têm suas energias abaixadas com a aplicação do campo, enquanto aqueles com $\mu=-$ têm as mesmas elevadas. Assim, elétrons com spin $\mu=-$ migram para a banda $\operatorname{com} \mu=+$ fazendo com que a largura dos estados ocupados com $\mu=+$ cresça em detrimento da diminuição da largura dos estado ocupados com $\mu=-$ (ver Fig. (2.1)). Para se espelhar ao máximo no procedimento de Wilson [2], que no entanto é para bandas simétricas, vamos redefinir as mesmas energias de forma que o nível de Fermi volte a ser $\epsilon_{F}=0$ em ambas as bandas (ver Fig. (2.2)). Como o procedimento de discretização que vamos descrever não depende do spin, vamos considerar somente 


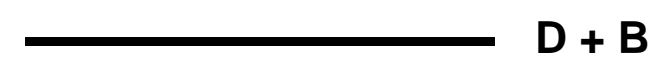

\section{D - B}
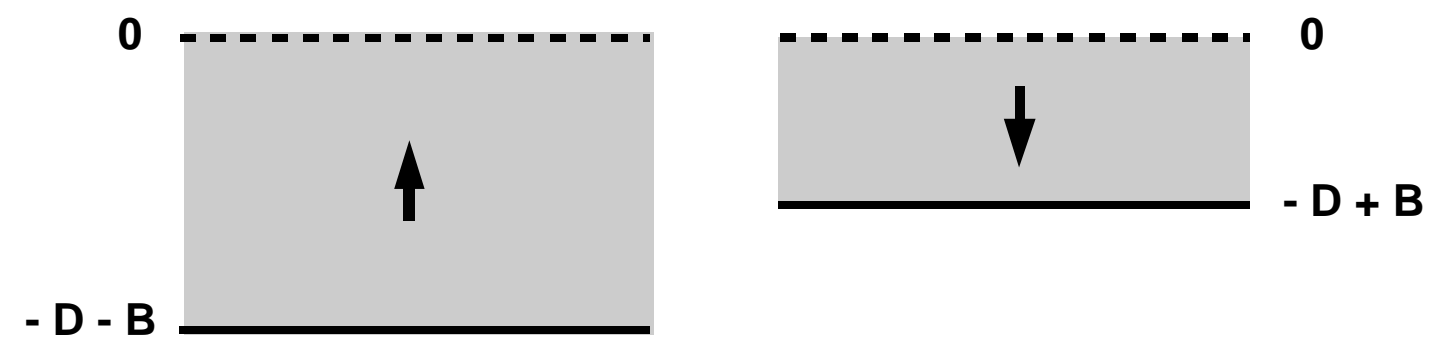

Figura 2.2: Aqui, as duas sub-bandas de spins opostos tem seus limites de energia redefinidos de tal forma que a energia de Fermi, $\epsilon_{F}$, da banda completa seja igual a zero.

o setor $\mu=+$. Ao final, poderemos acrescentar trivialmente o setor $\mu=-$ fazendo-se $B \rightarrow-B$, como se vê pela Fig. (2.2). Assim, começamos com o seguinte Hamiltoniano de condução com campo:

$$
H_{B C}=\sum_{\vec{k}}\left(\epsilon_{\vec{k}}-B\right) c_{\vec{k}}^{\dagger} c_{\vec{k}}
$$

Sem campo externo, a banda se extende de 0 a $2 D$, simetricamente em torno do nível de Fermi $\epsilon_{F}=D$. Com campo, todos os níveis ocupados sobem de $B$ e assim o nível de Fermi se posiciona em $\epsilon=D+B$. Para se espelhar ao máximo no procedimento de Wilson para bandas simétricas, vamos redefinir as energias de forma que o nível de Fermi volte a ser $\epsilon_{F}=0$, e assim, o fundo da banda está em $-D-B$ e o topo em $D-B$.

Para relações de dispersão isotrópicas, isto é, $\epsilon_{\vec{k}}=\epsilon_{|\vec{k}|} \equiv D \epsilon$, podemos trabalhar com operadores $c_{\epsilon}$ no lugar dos $c_{\vec{k}}$, onde a energia $\epsilon$ passa a ser medida em unidades de $D$ e assim,

$$
H_{B C}=D \int_{-1-B}^{1-B} \epsilon c_{\epsilon}^{\dagger} c_{\epsilon} d \epsilon
$$

onde para que tenhamos $\left\{c_{\epsilon}^{\dagger}, c_{\epsilon^{\prime}}\right\}=\delta\left(\epsilon-\epsilon^{\prime}\right)$ definimos $c_{k}=\sqrt{D} c_{\epsilon}$; o campo $B$ também passa a ser medido em unidades de $D$.

Quando usamos o GRN temos em mente determinar a contribuição do nível localizado (em 
geral na forma de uma impureza) à propriedade física. Para isso, a banda de condução sofre sucessivas transformações para torná-la numericamente tratável. Na forma acima temos um número infinito de estados (ou da ordem de $10^{23}$ ) o que inviabiliza qualquer cálculo numérico. Wilson, então, discretiza a banda de forma que uma dada energia $\epsilon$ está compreendida sempre entre $\Lambda^{-m-1} \leq|\epsilon| \leq \Lambda^{-m}$, para um $m$ inteiro e $\Lambda>1$ escolhido. Essa discretização introduz oscilações espúrias em qualquer propriedade termodinâmica quando expressa em função da temperatura. Para contornar isso, a Ref. [8] define a discretização a dois parâmetros, tal que $\Lambda^{-m-z-1} \leq|\epsilon| \leq \Lambda^{-m-z}$, para um $m$ inteiro, $\Lambda>1$ e $z>0$. Mostra-se que mesmo para grandes $\Lambda^{\prime} s$ como os do intervalo de 4 a 10 , médias com dois ou quatro valores de $z$ já suavizam curvas de entropia ou de suscetibilidade magnética a ponto das oscilações serem desprezíveis.

Para implementar a discretização a dois parâmetros e com campo magnético faremos uso de dois procedimentos complementares: um presente na tese de Luiz Nunes de Oliveira [11], que estuda o modelo de Vigman-Finkelshtein na presença de um campo (lá denominado de campo de carga), mas que utiliza apenas um parâmetro de discretização, o $\Lambda$; o outro procedimento, descrito na tese de Washington [12], descreve discretizações com dois parâmetros, mas sem campo externo.

A Fig. (2.3) ilustra a discretização logarítmica utilizada nesta tese: Para cada intervalo

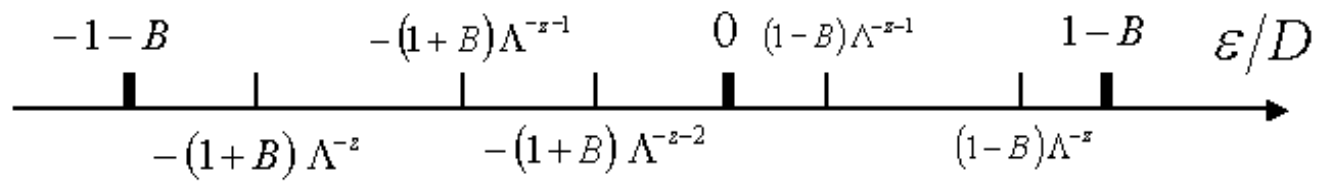

Figura 2.3: Discretização logarítmica da banda de condução segundo preceitua o GRN. Porém, foi introduzido o parâmetro de discretização $z$, além do campo magnético aplicado $B$.

definimos um conjunto de ondas planas que servirão de base para expandirmos os operadores $c_{\epsilon}$. Para o intervalo no topo da banda, $(1-B) \Lambda^{-z}<\epsilon / D<1-B$, e para o intervalo no fundo da banda, $-(1+B)<\epsilon / D<-(1+B) \Lambda^{-z}$, definimos um conjunto completo de funções com índice de Fourier 
$l$, semelhantemente ao que se faz no procedimento usual de Wilson [2], mas aqui generalizado para $z \neq 1[8]:$

$$
\psi^{ \pm}(\epsilon)=\frac{1}{\left(1-\Lambda^{-z}\right)^{1 / 2}} \frac{1}{(1 \mp B)^{1 / 2}} e^{ \pm \frac{2 \pi i}{1-\Lambda^{-z}} l \epsilon} .
$$

Para os outros intervalos da banda as funções de Fourier são:

$$
\psi_{m}^{ \pm}(\epsilon)=\frac{\Lambda^{\frac{m+z}{2}}}{\left(1-\Lambda^{-1}\right)^{1 / 2}} \frac{1}{(1 \mp B)^{1 / 2}} e^{ \pm \frac{2 \pi i \Lambda^{m+z}}{1-\Lambda^{-z}} l \epsilon}
$$

sendo o sinal superior para intervalos de energias positivas $(1-B) \Lambda^{-m-1-z}<\epsilon / D<(1-B) \Lambda^{-m-z}$, e o sinal inferior para os intervalos de energias negativas, $-(1+B) \Lambda^{-m-z}<\epsilon / D<-(1+B) \Lambda^{-m-1-z}$. Os coeficientes das exponenciais acima normalizam as funções à unidade.

Devemos agora projetar os operadores da banda de condução utilizando esse conjunto completo de funções ortonormais. No entanto, como sempre vamos considerar impurezas com simetria esférica, isto é, acopladas isotropicamente com a banda, os estados com $l \neq 0$ se desacoplam e formam um gás de elétrons livres que não contribuem para as propriedades da impureza ao sistema. Sendo assim, levamos em frente apenas os estados com $l=0$ e a expansão dos seguintes operadores $c_{\epsilon}$ nessa base define o conjunto de operadores discretos $\left\{a, a_{m}, b, b_{m}\right\}$, como coeficientes na expansão

$$
c_{\epsilon}=a \psi^{+}+b \psi^{-}+\sum_{m}\left(a_{m} \psi_{m}^{+}+b_{m} \psi_{m}^{-}\right)
$$

Em termos desses novos operadores a banda se escreve

$$
\begin{aligned}
H_{B C} & =\frac{1+\Lambda^{-z}}{2}\left[(1-B) a^{\dagger} a-(1+B) b^{\dagger} b\right] \\
& +\frac{1+\Lambda^{-1}}{2} \sum_{m=0}^{\infty} \Lambda^{-(m+z)}\left[(1-B) a_{m}^{\dagger} a_{m}-(1+B) b_{m}^{\dagger} b_{m}\right] \\
& \equiv \sum_{m=1}^{M} E_{m} d_{m}^{\dagger} d_{m}
\end{aligned}
$$

A última igualdade define os coeficientes $E_{m}(B)$ e os operadores discretos $d_{m}$. Estamos denotando por $M$ o limite superior da última soma, que é um número, em princípio, da ordem de $10^{23}$. 
O próximo passo consiste em definir um conjunto de novos operadores $\left\{f_{0}, f_{1}, \ldots, f_{n}\right\}$ de modo que o Hamiltoniano da banda de condução tome a forma

$$
H_{B C}=\sum_{n=0}^{M-2} t_{n}\left(f_{n}^{\dagger} f_{n+1}+H . c .\right)+\sum_{n=0}^{M-1} \zeta_{n} f_{n}^{\dagger} f_{n}
$$

onde o operador de partida $f_{0}$ é definido por

$$
f_{0}=\frac{1}{\sqrt{2}} \sum_{\vec{k}} c_{\vec{k}}=\frac{1}{\sqrt{2 D}} \int_{-D-B}^{D+B} c_{\epsilon} d \epsilon
$$

e que usando as Eq. (2.5), (2.6) e (2.7) se escreve como

$$
\begin{aligned}
f_{0} & =\sqrt{\frac{1-\Lambda^{-z}}{2}}\left[(1-B)^{1 / 2} a+(1+B)^{1 / 2} b\right] \\
& +\sqrt{\frac{1-\Lambda^{-1}}{2}} \sum_{m} \Lambda^{\frac{-(m+z)}{2}}\left[(1-B)^{1 / 2} a_{m}+(1+B)^{1 / 2} b_{m}\right] \\
& \equiv \sum_{m=1}^{M} \alpha_{m} d_{m}
\end{aligned}
$$

Esta última igualdade define os coeficientes $\alpha_{m}$. A razão fundamental para se definir o operador $f_{0}$ pela relação acima é que em geral a impureza interage com a banda via um termo da forma $\sum c_{\vec{k}}$. Para exemplos, ver Eq. (3.1) que define o Modelo de Nível Ressonante em que esse termo aparece na hibridização dos estados localizados com a banda; ver também a Eq. (4.1), que define o modelo de Falicov-Kimball, no qual esse operador aparece tanto no termo de espalhamento Coulombiano (termo proporcional a $G$ ) como no termo de hibridização (proporcional a $V$ ). Dessa forma, a impureza se acopla com a banda diretamente sempre via o perador $f_{0}$ e nele não há estados com índice de Fourier $l \neq 0$, justificando, assim, o abandono dos estados $c_{\epsilon, l \neq 0}$ da banda.

A motivação para se reescrever $H_{B C}$ na forma da Eq. (2.9) é que, como veremos, os coeficientes $t_{n}$ e $\zeta_{n}$ decrescem muito rapidamente conforme $n$ cresce, o que permite truncar as duas séries de $H_{B C}$, assim viabilizando um tratamento numérico do problema. Diferentemente do caso em que $B=0$, agora aparecem os coeficientes diagonais $\zeta_{n}$, que se anulam para $B=0$. Os coeficientes $t_{n}$ 
dependem de $B$ de tal forma que $t_{n}=\epsilon_{n}$ quando $B=0$, sendo $\epsilon_{n}$ os coeficientes tridiagonais usuais do GRN, iguais aos obtidos por Wilson no caso $z=1$.

Outro aspecto importante na definição de $f_{0}$ é que, sendo ele o único operador diretamente acoplado à impureza, os truncamentos em $H_{B C}$ não efetam esse acoplamento; por isso se diz que o GRN é não-perturbativo e a aproximação decorrente dos truncamentos é controlável, isto é, dependendo do sistema físico e também das facilidades computacionais pode-se manter poucos ou muitos estados após o truncamento.

Passemos, então, a determinar os coeficientes $t_{n}$ e $\zeta_{n}$. A representação matricial da Eq. (2.9), chamemos de $\left[H_{B C}^{(M)}\right]$, escreve-se da seguinte maneira na base dos operadores $f_{n}$ :

$$
\left[H_{B C}^{(M)}\right]=\left[\begin{array}{ccccc}
\zeta_{0} & t_{0} & 0 & \cdots & 0 \\
t_{0} & \zeta_{1} & t_{1} & \cdots & 0 \\
0 & t_{1} & \ddots & \ddots & \vdots \\
\vdots & \vdots & \ddots & \zeta_{M-2} & t_{M-2} \\
0 & 0 & \cdots & t_{M-2} & \zeta_{M-1}
\end{array}\right]_{M \times M}
$$

A esta matriz, podemos associar polinômios característicos $P_{N}(\zeta) \equiv \operatorname{det}\left(\left[H_{B C}^{N}\right]-I \zeta\right)$, onde $I$ é a matriz identidade de ordem $N$ e $N<M$. Por completeza, definamos $P_{0}(\epsilon) \equiv 1$ e os demais são obtidos assim:

$$
\begin{gathered}
P_{1}=\left(\zeta_{0}-\epsilon\right) P_{0} \\
P_{2}=\operatorname{det}\left[\begin{array}{cc}
\zeta_{0}-\epsilon & t_{0} \\
t_{0} & \zeta_{1}-\epsilon
\end{array}\right]=\left(\zeta_{0}-\epsilon\right)\left(\zeta_{1}-\epsilon\right)-t_{0}^{2} \\
=\left(\zeta_{1}-\epsilon\right) P_{1}-t_{0}^{2} P_{0}
\end{gathered}
$$




$$
\begin{aligned}
P_{3}= & \operatorname{det}\left[\begin{array}{ccc}
\zeta_{0}-\epsilon & t_{0} & 0 \\
t_{0} & \zeta_{1}-\epsilon & t_{1} \\
0 & t_{1} & \zeta_{2}-\epsilon
\end{array}\right]= \\
& \left(\zeta_{0}-\epsilon\right)\left(\zeta_{1}-\epsilon\right)\left(\zeta_{2}-\epsilon\right)-t_{0}^{2}\left(\zeta_{2}-\epsilon\right)-t_{1}^{2}\left(\zeta_{0}-\epsilon\right) \\
= & \left(\zeta_{2}-\epsilon\right)\left[\left(\zeta_{0}-\epsilon\right)\left(\zeta_{1}-\epsilon\right)-t_{0}^{2}\right]-t_{1}^{2}\left(\zeta_{0}-\epsilon\right)=\left(\zeta_{2}-\epsilon\right) P_{2}-t_{1}^{2} P_{1} .
\end{aligned}
$$

Esses poucos exempos já mostram que os polinômios característicos obedecem à relação de recorrência

$$
P_{N+1}=\left(\zeta_{N}-\epsilon\right) P_{N}-t_{N-1}^{2} P_{N-1}
$$

e são da forma

$$
P_{N}(\epsilon)=(-1)^{N}\left(\epsilon^{N}+\gamma_{1} \epsilon^{N-1}+\gamma_{2} \epsilon^{N-2}+\ldots+\gamma_{N} \epsilon^{0}\right)
$$

com $\gamma_{i}$ constantes independentes de $\epsilon$. Por inspeção verificamos facilmente que

$$
\gamma_{1}=-\sum_{n=0}^{N-1} \zeta_{n}
$$

relação que será importante para obtermos os coeficientes $\zeta_{n}$.

Queremos que os $P_{n}(\epsilon)$ sejam ortogonais segundo um peso $W(\epsilon)$, isto é,

$$
\left\langle P_{n} \mid P_{m}\right\rangle \equiv \int d \epsilon W(\epsilon) P_{n}(\epsilon) P_{m}(\epsilon)
$$

seja nulo para $n \neq m$, com $\int d \epsilon W(\epsilon)=1$.

Como $M$ é não nulo, temos que

$$
0=\left\langle P_{M} \mid P_{0}\right\rangle=\int d \epsilon W(\epsilon) P_{M}(\epsilon)
$$

Uma vez que $P_{M}\left(E_{m}\right)=0$, já que os $E_{m}$ são os autovalores da matriz $H_{B C}^{M}$ acima, para satisfazer a equação acima basta que $W$ seja da forma geral $W(\epsilon)=\sum_{m} g_{m}(\epsilon) \delta\left(\epsilon-E_{m}\right)$, com $g_{m}(\epsilon)$ uma função 
a ser determinada. ${ }^{1}$ Como $\int W d \epsilon=1$, vem que $\sum_{m} g_{m}\left(E_{m}\right)=1$. Mas de

$$
0=\left\langle P_{1} \mid P_{0}\right\rangle=\int d \epsilon\left(\zeta_{0}-\epsilon\right) \sum_{m} g_{m} \delta\left(\epsilon-E_{m}\right)=\sum_{m}\left(\zeta_{0}-E_{m}\right) g_{m}\left(E_{m}\right)
$$

temos que

$$
\zeta_{0}=\sum_{m=1}^{M} g_{m}\left(E_{m}\right) E_{m}
$$

Para achar a função $g_{m}\left(E_{m}\right)$ comparemos o Hamiltoniano $H_{B C}$ ora escrito na base dos $f_{n}$ e ora escrito na base dos $d_{m}$. Da Eq.(2.9) vem que

$$
H_{B C}=\left(\zeta_{0} f_{0}^{\dagger}+t_{0} f_{1}^{\dagger}\right) f_{0}+\text { termos sem } f_{0}
$$

A definição anterior, $f_{0}=\sum_{1}^{M} \alpha_{m} d_{m}$ implica que $d_{m}=\alpha_{m} f_{0}+$ termos sem $f_{0}$. Substituindo esse $d_{m}$ na Eq. (2.8) vem que

$$
H_{B C}=\sum_{m=1}^{M} E_{m} \alpha_{m} d_{m}^{\dagger} f_{0}+\operatorname{termos} \operatorname{sem} f_{0} .
$$

Comparando essas duas últimas equações temos

$$
\zeta_{0} f_{0}^{\dagger}+t_{0} f_{1}^{\dagger}=\sum_{m=0}^{M} E_{m} \alpha_{m} d_{m}^{\dagger}
$$

que relaciona os operadores $d_{m}$ e $f_{0}$ com $t_{0}$. Comutando ambos os lados com $f_{0}$ temos

$$
\zeta_{0}=\sum_{m=1}^{M} \alpha_{m}^{2} E_{m}
$$

de maneira que comparando com a Eq. (2.22) achamos $g_{m}=\alpha_{m}^{2}$ e assim,

$$
W(\epsilon)=\sum_{m=1}^{M} \alpha_{m}^{2} \delta\left(\epsilon-E_{m}\right) \text {. }
$$

Comutando-se cada membro com seus respectivos conjugados, a Eq. (2.25) fornece ${ }^{2}$

$$
t_{0}^{2}=\sum_{m} \alpha_{m}^{2} E_{m}^{2}-\zeta_{0}^{2}
$$

\footnotetext{
${ }^{1}$ a nossa obtenção de $W$ difere daquela apresentada na Ref. [12]

${ }^{2}$ Da igualdade $0=\left\langle P_{2} \mid P_{0}\right\rangle$ tiramos que $t_{0}^{2}=\int d \epsilon W(\epsilon) \epsilon^{2}-\zeta_{0}^{2}$, que também implica em $g_{m}=\alpha_{m}^{2}$; também verificamos explicitamente que essa conclusão é consistente com $0=\left\langle P_{3} \mid P_{0}\right\rangle$.
} 
Então, $\zeta_{0}$ e $t_{0}$ dependem dos momentos de energia $\phi_{1}$ e $\phi_{2}$ definidos para um $n$ qualquer por

$$
\phi_{n}=\sum_{m=0}^{M} \alpha_{m}^{2} E_{m}^{n} .
$$

Da igualdade $0=\left\langle P_{N} \mid P_{0}\right\rangle$, vem que

$$
0=\int d \epsilon W(\epsilon) P_{N}(\epsilon) P_{0}=\int d \epsilon W(\epsilon)(-1)^{N}\left(\epsilon^{N}+\gamma_{1} \epsilon^{N-1}+\ldots+\gamma_{N}\right)
$$

que substituindo $W(\epsilon)$ e reescrevendo em termos dos momentos $\phi_{n}$ fica

$$
0=\phi_{N}+\gamma_{1} \phi_{N-1}+\ldots+\gamma_{N} \phi_{0}
$$

Como $P_{N}$ é combinação linear de $\epsilon^{j \leq N}$, então, $\epsilon^{n<N}$ é combinação apenas dos polinômios $P_{0}, P_{1}, \ldots, P_{n<N}$, não entrando $P_{N}$ nessa combinação linear. Assim, das igualdades $\left\langle P_{N} \mid \epsilon\right\rangle=0$, $\left\langle P_{N} \mid \epsilon^{2}\right\rangle=0, \ldots,\left\langle P_{N} \mid \epsilon^{N-1}\right\rangle=0$, também reescritas em termos dos momentos $\phi_{n}$, chegamos ao sistema linear de equações (onde os $\gamma_{i}$ são as incógnitas):

$$
\begin{aligned}
\phi_{N}+\gamma_{1} \phi_{N-1}+\ldots+\gamma_{N} \phi_{0} & =0 \\
\phi_{N+1}+\gamma_{1} \phi_{N}+\ldots+\gamma_{N} \phi_{1} & =0 \\
& \vdots \\
\phi_{2 N-1}+\gamma_{1} \phi_{2 N-2}+\ldots+\gamma_{N} \phi_{N-1} & =0
\end{aligned}
$$

Coloquemos esse sistema na forma matricial:

$$
\left[\begin{array}{cccc}
\phi_{0} & \ldots & \phi_{N-2} & \phi_{N-1} \\
\phi_{1} & \ldots & \phi_{N-1} & \phi_{N} \\
\vdots & \vdots & \vdots & \vdots \\
\phi_{N-1} & \ldots & \phi_{2 N-3} & \phi_{2 N-2}
\end{array}\right]\left[\begin{array}{c}
\gamma_{N} \\
\gamma_{N-1} \\
\vdots \\
\gamma_{1}
\end{array}\right]=-\left[\begin{array}{c}
\phi_{N} \\
\phi_{N+1} \\
\vdots \\
\phi_{2 N-1}
\end{array}\right]
$$

Trata-se de um sistema não homogêneo de equações lineares com relação aos $\gamma_{i}$ 's. Usando a regra de Cramer, achamos que

$$
\gamma_{1}=-\frac{\widetilde{\Delta}_{N}}{\Delta_{N}},
$$


onde

$$
\widetilde{\Delta}_{N}=\operatorname{det}\left[\begin{array}{cccc}
\phi_{0} & \ldots & \phi_{N-2} & \phi_{N} \\
\phi_{1} & \ldots & \phi_{N-1} & \phi_{N+1} \\
\vdots & \vdots & \vdots & \vdots \\
\phi_{N-1} & \ldots & \phi_{2 N-3} & \phi_{2 N-1}
\end{array}\right]
$$

$\mathrm{e}$

$$
\Delta_{N}=\operatorname{det}\left[\begin{array}{cccc}
\phi_{0} & \ldots & \phi_{N-2} & \phi_{N-1} \\
\phi_{1} & \ldots & \phi_{N-1} & \phi_{N} \\
\vdots & \vdots & \vdots & \vdots \\
\phi_{N-1} & \ldots & \phi_{2 N-3} & \phi_{2 N-2}
\end{array}\right] \text {. }
$$

Por outro lado, sendo $\gamma_{1}$ o coeficiente do termo $\epsilon^{N-1}$, então, pela Eq. (2.18),

$$
\sum_{i=0}^{N-1} \zeta_{n}=\frac{\widetilde{\Delta}_{N}}{\Delta_{N}}
$$

e, portanto, segue imediatamente que os coeficientes diagonais podem ser obtidos pela fórmula

$$
\zeta_{N}=\frac{\tilde{\Delta}_{N+1}}{\Delta_{N+1}}-\frac{\tilde{\Delta}_{N}}{\Delta_{N}}, \quad \text { para } \quad N \geq 1
$$

Para determinar os coeficientes codiagonais $t_{N}$, usamos a recorrência $P_{N+1}=\left(\zeta_{N}-\epsilon\right) P_{N}-t_{N-1}^{2} P_{N-1}$. Então, o produto escalar $\left\langle P_{N+1} \mid P_{N-1}\right\rangle=0$ fornece

$$
t_{N-1}^{2}=\frac{\left\langle P_{N} \mid P_{N}\right\rangle}{\left\langle P_{N-1} \mid P_{N-1}\right\rangle}
$$

onde usamos que $\epsilon P_{N-1}=-P_{N}+\gamma_{N} P_{0}$. Dessa equação escrevemos

$$
\left\langle P_{N} \mid P_{N}\right\rangle=t_{N-1}^{2}\left\langle P_{N-1} \mid P_{N-1}\right\rangle=t_{N-1}^{2} t_{N-2}^{2}\left\langle P_{N-2} \mid P_{N-2}\right\rangle=\ldots=\prod_{n=0}^{N-1} t_{n}^{2}
$$

Por outro lado,

$$
\left\langle P_{N} \mid P_{N}\right\rangle=(-1)^{N} \int d \epsilon W(\epsilon) P_{N}(\epsilon)\left(\epsilon^{N} \gamma_{1} \epsilon^{N-1}+\gamma_{2} \epsilon_{N-2}+\ldots+\gamma_{N} \epsilon^{0}\right)=(-1)^{N}\left\langle P_{N} \mid \epsilon^{N}\right\rangle,
$$


visto que $P_{N}(\epsilon)$ é ortogonal a $\epsilon^{n}$ se $n<N$. Então,

$$
\left\langle P_{N} \mid P_{N}\right\rangle=\int d \epsilon W(\epsilon)\left(\epsilon^{N}+\gamma_{1} \epsilon^{N-1}+\ldots+\gamma_{N}\right) \epsilon^{N}=\phi_{2 N}+\gamma_{1} \phi_{2 N-1}+\ldots+\gamma_{N} \phi_{N}
$$

Portanto,

$$
\phi_{2 N}+\gamma_{1} \phi_{2 N-1}+\ldots+\gamma_{N} \phi_{N}=\prod_{n=0}^{N-1} t_{n}^{2}
$$

Adicionando esta equação àquelas do sistema não homogêneo anterior ficamos com

$$
\left[\begin{array}{ccccc}
\phi_{0} & \ldots & \phi_{N-2} & \phi_{N-1} & 0 \\
\phi_{1} & \ldots & \phi_{N-1} & \phi_{N} & 0 \\
\vdots & \vdots & \vdots & \vdots & 0 \\
\phi_{N-1} & \ldots & \phi_{2 N-3} & \phi_{2 N-2} & 0 \\
\phi_{N} & \ldots & \phi_{2 N-2} & \phi_{2 N-1} & -1
\end{array}\right]\left[\begin{array}{c}
\gamma_{N} \\
\gamma_{N-1} \\
\vdots \\
\gamma_{1} \\
\prod_{n=0}^{N-1} t_{n}^{2}
\end{array}\right]=-\left[\begin{array}{c}
\phi_{N} \\
\phi_{N+1} \\
\vdots \\
\phi_{2 N-1} \\
\phi_{2 N}
\end{array}\right]
$$

Usando a Regra de Cramer temos

$$
\prod_{n=0}^{N-1} t_{n}^{2}=-\frac{\left[\begin{array}{ccccc}
\phi_{0} & \ldots & \phi_{N-2} & \phi_{N-1} & \phi_{N} \\
\phi_{1} & \ldots & \phi_{N-1} & \phi_{N} & \phi_{N+1} \\
\vdots & \vdots & \vdots & \vdots & \vdots \\
\phi_{N-1} & \ldots & \phi_{2 N-3} & \phi_{2 N-2} & \phi_{2 N-1} \\
\phi_{N} & \ldots & \phi_{2 N-2} & \phi_{2 N-1} & \phi_{2 N}
\end{array}\right]}{\operatorname{det}\left[\begin{array}{ccccc}
\phi_{0} & \ldots & \phi_{N-2} & \phi_{N-1} & 0 \\
\phi_{1} & \ldots & \phi_{N-1} & \phi_{N} & 0 \\
\vdots & \vdots & \vdots & \vdots & 0 \\
\phi_{N-1} & \ldots & \phi_{2 N-3} & \phi_{2 N-2} & 0 \\
\phi_{N} & \ldots & \phi_{2 N-2} & \phi_{2 N-1} & -1
\end{array}\right]}
$$

Note que o determinante no numerador é um daqueles que usamos anteriormente, $\Delta_{N+1}$, enquanto o do denominador é o próprio $\Delta_{N}$, porém com o sinal trocado (usando método dos cofatores com a 
última coluna obtém-se facilmente este resultado). Então, chegamos a

$$
\prod_{n=0}^{N-1} t_{n}^{2}=\frac{\Delta_{N+1}}{\Delta_{N}}
$$

Disso resulta imediatamente os coeficientes codiagonais

$$
t_{N}=\frac{\sqrt{\Delta_{N+2} \Delta_{N}}}{\Delta_{N+1}}, \quad \text { para } \quad N \geq 1
$$

\subsubsection{Os coeficientes $\zeta_{0}$ e $t_{0}$}

Para $N=0$, temos $\zeta_{0}=\phi_{1}$ e $t_{0}^{2}=\phi_{2}-\zeta_{0}^{2}$. Façamos explicitamente o cálculo para $\zeta_{0}$ e $t_{0}$ :

$$
\begin{aligned}
\zeta_{0} & =\sum_{m=1}^{M} E_{m} \alpha_{m}^{2}=\left(\frac{1-\Lambda^{-z}}{2}\right)\left(\frac{1+\Lambda^{-z}}{2}\right)\left[(1-B)^{2}-(1+B)^{2}\right] \\
& +\sum_{m=1}^{M}\left\{\left(\frac{1-\Lambda^{-1}}{2}\right)\left(\frac{1+\Lambda^{-1}}{2}\right)\left[(1-B)^{2}-(1+B)^{2}\right] \Lambda^{-2(m+z)}\right\}=-B,
\end{aligned}
$$

onde usamos que $\sum_{m=1}^{M \rightarrow \infty} \Lambda^{\alpha m}=1 /\left(1-\Lambda^{\alpha}\right)$. E para o codiagonal:

$$
\begin{aligned}
t_{0}^{2} & =\sum_{m=1}^{M} E_{m}^{2} \alpha_{m}^{2}-\zeta_{0}^{2}=\left(\frac{1+\Lambda^{-z}}{2}\right)^{2}\left(\frac{1-\Lambda^{-z}}{2}\right)\left[(1-B)^{3}+(1+B)^{3}\right] \\
& +\sum_{m=1}^{M}\left(\frac{1+\Lambda^{-1}}{2}\right)^{2}\left(\frac{1-\Lambda^{-1}}{2}\right) \Lambda^{-3(m+z)}\left[(1-B)^{3}+(1+B)^{3}\right]-B^{2} \\
& =\left(\frac{1+\Lambda^{-z}}{2}\right)^{2}\left(\frac{1-\Lambda^{-z}}{2}\right) 2\left(1+3 B^{2}\right) \\
& +\left(\frac{1+\Lambda^{-1}}{2}\right)^{2}\left(\frac{1-\Lambda^{-1}}{2}\right) 2\left(1+3 B^{2}\right) \frac{\Lambda^{-3 z}}{1-\Lambda^{-3}}-B^{2} .
\end{aligned}
$$

Quando $B=0$ e $z=1$, temos:

$$
t_{0}^{2}=\left(\frac{1+\Lambda^{-1}}{2}\right)^{2} \frac{1+\Lambda^{-1}}{1-\Lambda^{-3}}
$$

que corresponde exatamente à expressão analítica obtida por Wilson [2]. Quando $B \neq 0$, mas $z=1$,

$$
t_{0}^{2}=\left[1-B^{2}\left(\frac{1-\Lambda^{-1}}{1+\Lambda^{-1}}\right)^{2}\right] t_{0}^{2}(B=0)
$$

concordando com a expressão encontrada na Ref.[11]. 


\subsubsection{Justificando Truncamento para $N$ finito}

Na Eq. (2.9) as somas se extendem até $M \rightarrow \infty$. Na prática essas séries podem ser truncadas num $N$ finito por duas razões:

1. a primeira é técnica: os coeficientes $t_{n}$ e $\zeta_{n}$ vão a zero rapidamente conforme $n$ cresce. Para $z=1$ temos que $[11]$

$$
t_{n}(z=1)=\epsilon_{n}\left[1-B^{2}\left(\frac{1-\Lambda^{-n-1}}{1+\Lambda^{-n-1}}\right)^{2}\right]^{1 / 2}
$$

onde $\epsilon_{n}$ é o coeficiente achado por Wilson, [2], que para $n$ grande vai como $\Lambda^{-n / 2} .{ }^{3}$ Isso garante a convergência da primeira série da Eq. (2.9). Para $z \neq 1$ também é sabido que $t_{n} \approx \Lambda^{-n / 2+1-z}$ [8]. Quanto aos coeficientes $\zeta_{n}$, para $z=1$ temos que [11]

$$
\zeta_{n}=-4 B^{2} \frac{\Lambda^{-n}}{\left(1+\Lambda^{-n}\right)\left(1+\Lambda^{-n-1}\right)},
$$

que converge ainda mais rápido a zero, garantindo o truncamente da segunda série da Eq. (2.9). Para $z \neq 1$ não há uma expressão para $\zeta_{n}$, mesmo para $n$ grande. Analisando os coeficientes $\zeta_{n}$ para diversos valores de $\Lambda, z$ e $B$, empiricamente chegamos à seguinte expressão assintótica que reproduz $\zeta_{n}$ com menos de $1 \%$ de erro já para $n$ tipicamente maior que apenas 6 :

$$
\zeta_{n} \approx-4 B^{2} \frac{\Lambda^{-n}}{\left(1+\Lambda^{-n}\right)\left(1+\Lambda^{-n-1}\right)} \Lambda^{|z-1| /\left[\frac{2}{5}\left(z-\frac{3}{4}\right)+\frac{2}{3}\right]} \cdot \operatorname{sinal}(z-1)
$$

O denominador do expoente de $\Lambda$ mais à direita foi obtido interpolando-se linearmente os valores de $\zeta_{n}$ calculados em $z=0.75$ e $z=1.25$, que são dois valores típicos utilizados na prática.

2. a segunda razão é de teor físico: truncando num determinado $N$ inclue-se escalas de energia da ordem de $t_{N} \approx \Lambda^{-N / 2}$. Se desejamos estudar excitações dessa ordem (por exemplo, para temperaturas tais que $\left.k_{B} T \approx \Lambda^{-N / 2} D\right)$, escalas menores serão irrelevantes e assim não há necessidade de estender a soma além desse determinado $N$.

\footnotetext{
${ }^{3} \mathrm{O}$ coeficiente exato determinado por Wilson é $\epsilon_{n} \equiv t_{n}(z=1)=\frac{1+\Lambda^{-1}}{2} \frac{1-\Lambda^{-n-1}}{\sqrt{\left(1-\Lambda^{-2 n-1}\right)\left(1-\Lambda^{-2 n-3}\right)}} \Lambda^{-n / 2}$.
} 
Devido à forte propagação de erros de arredondamento intrínsecos a este procedimento, escrevemos um programa em MAPLE com precisão de 200 casas decimais para obtermos valores confiáveis para $t_{n}$ e $\zeta_{n}$. Assim mesmo, para $N$ grande - tipicamente 15 - é melhor utilizar as fórmulas assintóticas acima do que tentar determinar numericamente os coeficientes. Antes de conhecermos o limite assintótico de $\zeta_{n}$ dado acima, simplesmente adotava-se $\zeta_{n>>1} \rightarrow 0$. Isso se justificava, no entanto, pelo fato de $\zeta_{n}$ ir a zero muito rapidamente com $n$.

\subsubsection{Por que discretizar banda + campo juntos}

Por último, queremos responder à seguinte possível indagação que poderíamos ter feito logo no início deste Capítulo: por que, mesmo com campo magnético, não seguimos a prescrição usual do GRN (mas ainda com $z \neq 1$ ), isto é, por que não discretizamos somente o termo da banda de condução, $\sum_{k} \epsilon_{k} c_{k}^{\dagger} c_{k}$, chegamos a uma base discreta $f_{n}$ (não os $f_{n}$ anteriores) e depois reescrevemos o termo de campo, $B \sum_{k} c_{k}^{\dagger} c_{k}$ ? Se assim fosse feito, o termo de campo seria dado por $B \sum_{n} f_{n}^{\dagger} f_{n}$, e não poderia ser truncado já que não tem um coeficiente convergindo a zero para $n$ grande. Isso foi indício suficientemente forte para suspeitarmos de que não estaríamos lidando corretamente com as escalas introduzidas pelo GRN caso introduzíssemos o campo dessa forma: é imprescindível ao GRN que lidemos com uma base de operadores discretos passível de ser truncada.

\subsubsection{Recuperando as bandas com spin $\mu= \pm$}

Para descrevermos bandas com spin $\uparrow$ e $\downarrow$ usamos que os coeficientes codiagonais $t_{n}(B)$ são pares em $B$, isto é, $t_{n}(-B)=t_{n}(B)$. Já os coeficientes diagonais $\zeta_{n}(B)$ são ímpares, ou seja, $\zeta_{n}(-B)=-\zeta_{n}(B)$. Sendo assim, a Eq. (2.9) fica

$$
H_{B C}=\sum_{n=0}^{M-2} t_{n}(B)\left(f_{n \uparrow}^{\dagger} f_{n+1 \uparrow}+f_{n \downarrow}^{\dagger} f_{n+1 \downarrow}+h . c .\right)+\sum_{n=0}^{M-1} \zeta_{n}(B)\left(f_{n \uparrow}^{\dagger} f_{n \uparrow}-f_{n \downarrow}^{\dagger} f_{n \downarrow}\right) .
$$

Além do argumento exposto acima, deve-se levar em conta que a implementação do GRN à 
banda assimétrica, tal como feita neste capítulo, pode abranger casos além da que é originada pelo campo magnético.

De fato, nos próximos capítulos trataremos da aplicação desse procedimento a dois modelos: o de Nível Ressonante e o de Falicov-Kimball, sendo que o agente externo que gera a assimetria não é o campo magnético, mas sim o "campo de carga". 


\section{Capítulo 3}

\section{Modelo de Nível Ressonante}

O Modelo de Nível Ressonante (MNR) [13] consiste em um nível localizado, de energia $E_{d}$, que se hibridiza com o contínuo da banda de condução de um metal. O Hamiltoniano que representa esse modelo é

$$
H=\sum_{\vec{k}, \sigma} \epsilon_{k} c_{\vec{k}, \sigma}^{\dagger} c_{\vec{k}, \sigma}+E_{d} \sum_{\sigma} d_{\sigma}^{\dagger} d_{\sigma}+V \sum_{\vec{k}, \sigma}\left(c_{\vec{k}, \sigma}^{\dagger} d_{\sigma}+\text { h.c. }\right),
$$

onde $c_{\vec{k}, \sigma}$ aniquila um elétron da banda com momento $\vec{k}$ e spin $\sigma$, enquanto $d_{\sigma}$ aniquila um elétron localizado. A hibridização é representada pelo termo proporcional a $V$, que é responsável pelo aparecimento de uma ressonância de largura $\Delta \approx \pi \rho V^{2}$ na banda, sendo $\rho$ a densidade de estados no nível de Fermi. Sendo o Hamiltoniano quadrático, é diagonalizável exatamente (pelo menos numericamente) e assim serve de laboratório teórico para se estudar sistemas em que haja uma impureza localizada em meio a um mar de elétrons.

Esse simples modelo tem sua importância na capacidade de abordar e ser ponto de partida para discussão de fenômenos importantes. Por exemplo, a anti-ressonância de Fano, que é resultado da interferência destrutiva entre duas amplitudes quânticas (uma, resultado da excitação do elétron localizado direto para a banda, e outra, da excitação desse elétron localizado com um estado misto banda-nível devido à hibridização). Ele também fornece uma descrição quantitativa bastante boa do 
calor específico do modelo de Kondo para impureza magnética.

Situações mais realistas exigem em que se incluam correlações no nível localizado, advindas de repulsão Coulombiana entre os elétrons. Quando o termo de correlação $U n_{d, \uparrow} n_{d, \downarrow}, \operatorname{com} n_{d, \sigma}=d_{\sigma}^{\dagger} d_{\sigma}$, é incluso no MNR temos o famoso modelo de Anderson que, sendo não quadrático, demanda grande esforço para ser diagonalizado. Em baixas temperaturas, o modelo de Anderson recai no modelo de Kondo para impureza magnética.

Aplicamos a discretização descrita anteriormente ao Modelo de Nível Ressonante (MNR) na presença de um campo magnético definido por:

$$
H=\sum_{k, \mu= \pm}\left(\epsilon_{k}-\mu B\right) c_{k, \mu}^{\dagger} c_{k, \mu}+E_{d} \sum_{\mu= \pm} d_{\mu}^{\dagger} d_{\mu}-B \sum_{\mu= \pm} \mu d_{\mu}^{\dagger} d_{\mu}+V \sum_{k, \mu= \pm}\left(c_{k, \mu}^{\dagger} d_{\mu}+\text { H.c. }\right),
$$

onde os níveis de impureza podem ser vazios, unicamente ocupados com energia $E_{d}(\operatorname{com} \operatorname{spin} \uparrow$ ou $\downarrow$ ) ou duplamente ocupado com energia $2 E_{d}$. Com campo magnético, o nível unicamente ocupado sofre efeito Zeeman e é separado em dois: um com energia $E_{d}-B$ e outro com energia $E_{d}+B$ (lembremo-nos de que convencionamos $B \equiv \frac{g \mu_{B}}{2} B_{z}$ ), conforme ilustra a Fig. (3.1). Os níveis da impureza hibridizamse com a banda de condução através de $V$, o qual confere aos níveis unicamente ocupados uma largura $\Delta=\pi V^{2}$

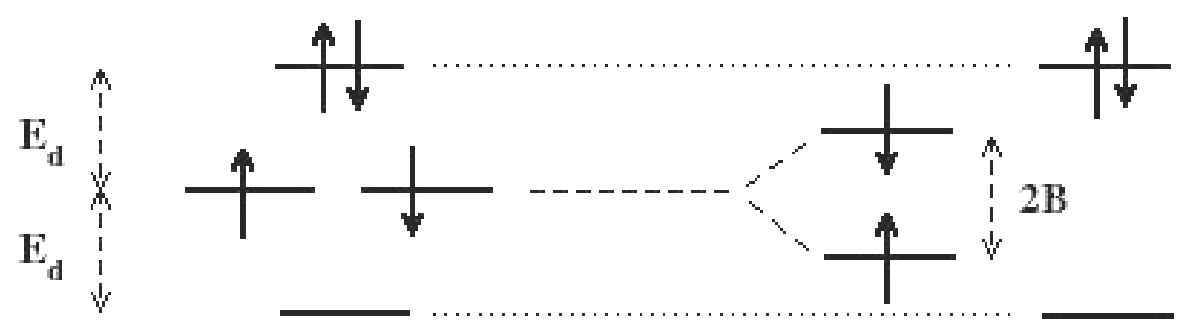

Figura 3.1: Diagrama de níveis do MNR. Os níveis unicamente ocupados apresentam largura devido à hibridização com a banda de condução. O campo magnético quebra a degenescência destes níveis.

Em termos do GRN, este Hamiltoniano se transforma seguindo as prescrições do Cap. 2. O primeiro termo da Eq. (3.2) é idêntico ao termo da Eq. (2.3), para cada canal de spin $\mu= \pm$. Assim, 
ele é transformado no $H_{B C}$ dado pela Eq. (2.52). Já o operador $\sum_{k} c_{k}^{\dagger}$ é transformado em

$$
f_{0}=\frac{1}{\sqrt{2 D}} \int_{-D-B}^{D+B} c_{\epsilon} d \epsilon
$$

após linearização da banda. Assim, de $H$ chegamos a $H_{N}$ dado por

$$
\begin{aligned}
H_{N} & =\Lambda^{\frac{N-1}{2}+z-1}\left\{\sum_{n=0}^{N-1} t_{n}(B)\left(f_{n+1, \uparrow}^{\dagger} f_{n, \uparrow}+f_{n+1, \downarrow}^{\dagger} f_{n, \downarrow}\right)+\sum_{n=0}^{N-1} \zeta_{n}(B)\left(f_{n, \uparrow}^{\dagger} f_{n, \uparrow}-f_{n, \downarrow}^{\dagger} f_{n, \downarrow}\right)\right. \\
& \left.+\widetilde{E}_{d} \sum_{\mu} d_{\mu}^{\dagger} d_{\mu}-\widetilde{B} \sum_{\mu} \mu d_{\mu}^{\dagger} d_{\mu}+\widetilde{V} \sum_{\mu}\left(f_{0, \mu}^{\dagger} d_{\mu}+\text { h.c. }\right)\right\},
\end{aligned}
$$

onde $\widetilde{E}_{d}=\frac{2}{1+\Lambda^{-1}} E_{d}, \quad \widetilde{V}=\frac{2}{1+\Lambda^{-1}} V$ e $\widetilde{B}=\frac{2}{1+\Lambda^{-1}} B$. Por conveniência numérica, introduziu-se o coeficiente multiplicativo $\Lambda^{\frac{N-1}{2}+z-1}$; isso torna os menores elementos de matriz de $H_{N}$ da ordem da unidade. O Hamiltoniano $H$ é recuperado fazendo-se

$$
\lim _{N \rightarrow \infty} \frac{1+\Lambda^{-1}}{2} \Lambda^{-\frac{N-1}{2}-z+1} H_{N}
$$

Podemos escrever $H_{N}$ como a soma de dois Hamiltonianos que comutam entre si: $H_{N}=$ $H_{N \uparrow}+H_{N \downarrow}$, sendo que

$$
\begin{aligned}
H_{N \uparrow} & \equiv \Lambda^{\frac{N-1}{2}+z-1} \sum_{n=0}^{N-1}\left[t_{n}(B) f_{n+1, \uparrow}^{\dagger} f_{n, \uparrow}+\zeta_{n}(B) f_{n, \uparrow}^{\dagger} f_{n, \uparrow}\right] \\
& +\left(\widetilde{E}_{d}-\widetilde{B}\right) d_{\uparrow}^{\dagger} d_{\uparrow}+\widetilde{V}\left(f_{0, \uparrow}^{\dagger} d_{\uparrow}+\text { h.c. }\right)
\end{aligned}
$$

e

$$
\begin{aligned}
H_{N \downarrow} & \equiv \Lambda^{\frac{N-1}{2}+z-1} \sum_{n=0}^{N-1}\left[t_{n}(B) f_{n+1, \downarrow}^{\dagger} f_{n, \downarrow}-\zeta_{n}(B) f_{n, \downarrow}^{\dagger} f_{n, \downarrow}\right] \\
& +\left(\widetilde{E}_{d}+\widetilde{B}\right) d_{\downarrow}^{\dagger} d_{\downarrow}+\widetilde{V}\left(f_{0, \downarrow}^{\dagger} d_{\downarrow}+\text { h.c. }\right) .
\end{aligned}
$$


Na base dos estados $\left\{d_{\sigma}, f_{0, \sigma}, f_{1, \sigma}, \ldots, f_{N, \sigma}\right\}$ esses Hamiltonianos são representados pelas matrizes:

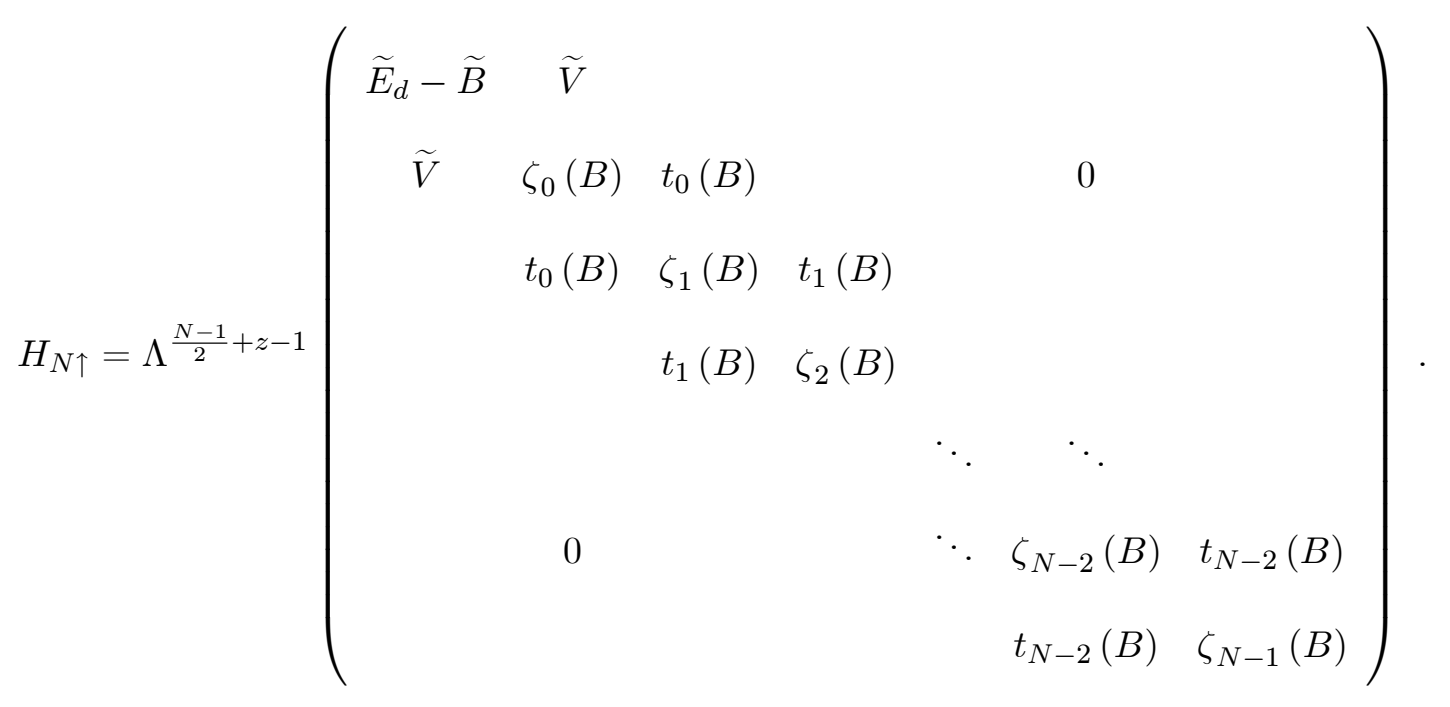

e

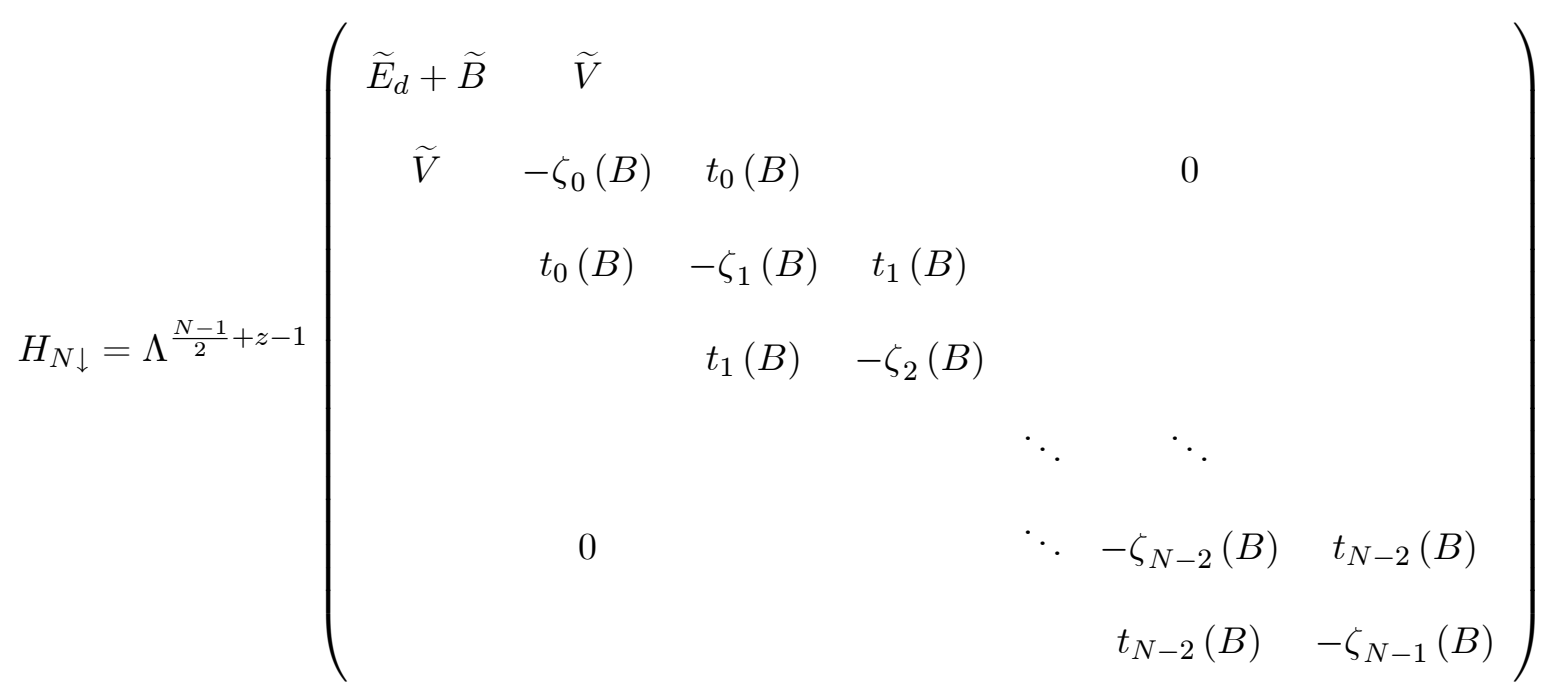

Como $\left[H_{N \uparrow}, H_{N \downarrow}\right]=0$, a função de partição pode ser escrita como:

$$
Z=\prod_{n}\left(1+e^{-\beta \epsilon_{n}^{\uparrow}}\right) \prod_{n}\left(1+e^{-\beta \epsilon_{n}^{\downarrow}}\right) \equiv Z_{\uparrow} Z_{\downarrow}
$$

sendo $\epsilon_{n}^{\uparrow}$ e $\epsilon_{n}^{\downarrow}$ autovalores de $H_{N \uparrow}$ e $H_{N \downarrow}$, respectivamente.

Assim, a energia livre é dada por $F=-k_{B} T\left(\ln Z_{\uparrow}+\ln Z_{\downarrow}\right)$, e podemos calcular a magnetização usando $M=-\partial F / \partial B_{z}$ :

$$
\frac{M}{g \mu_{B}}=-\frac{1}{2} \sum_{n}\left[\frac{e^{-\beta \epsilon_{n}^{\downarrow}}}{1+e^{-\beta \epsilon_{n}^{\downarrow}}}-\frac{e^{-\beta \epsilon_{n}^{\uparrow}}}{1+e^{-\beta \epsilon_{n}^{\uparrow}}}\right],
$$


onde $\beta \equiv 1 / k_{B} T$. Logo, a suscetibilidade magnética, definida por $\chi=\partial M / \partial B_{z}$, expressa-se como:

$$
\frac{k_{B} T \chi}{g \mu_{B}}=\frac{1}{4} \sum_{n}\left[\frac{e^{-\beta \epsilon_{n}^{\uparrow}}}{\left(1+e^{-\beta \epsilon_{n}^{\uparrow}}\right)^{2}}+\frac{e^{-\beta \epsilon_{n}^{\downarrow}}}{\left(1+e^{-\beta \epsilon_{n}^{\downarrow}}\right)^{2}}\right] .
$$

Para a entropia, $S=-\partial F / \partial T$, temos:

$$
\frac{S}{k_{B}}=\beta \sum_{n}\left[\epsilon_{n}^{\uparrow} \frac{e^{-\beta \epsilon_{n}^{\uparrow}}}{\left(1+e^{-\beta \epsilon_{n}^{\uparrow}}\right)^{2}}+\epsilon_{n}^{\downarrow} \frac{e^{-\beta \epsilon_{n}^{\downarrow}}}{\left(1+e^{-\beta \epsilon_{n}^{\downarrow}}\right)^{2}}\right]
$$

e para o calor específico, $C=T \partial S / \partial T$, temos:

$$
\frac{C}{k_{B}}=\beta^{2} \sum_{n}\left[\epsilon_{n}^{\uparrow 2} \frac{e^{-\beta \epsilon_{n}^{\uparrow}}}{\left(1+e^{-\beta \epsilon_{n}^{\uparrow}}\right)^{2}}+\epsilon_{n}^{\downarrow 2} \frac{e^{-\beta \epsilon_{n}^{\downarrow}}}{\left(1+e^{-\beta \epsilon_{n}^{\downarrow}}\right)^{2}}\right] .
$$

\subsection{Expressões analíticas para o MNR}

Para efeito de comparação dos resultados por nós computados com aqueles presentes na literatura usaremos o artigo de Schotte e Schotte [13]. No entanto, nossa abordagem é para um sistema com impureza do tipo Anderson (embora com correlação $U=0$ ), isto é, podendo estar num nível vazio, unicamente ocupado (com spin $\uparrow$ ou $\downarrow$ ) de energia $E_{d} \pm B$ ou duplamente ocupado com energia $2 E_{d}$ (no modelo de Anderson seria $2 E_{d}+U$ ). Já nos artigos da Ref. [13] foram excluídos os níveis vazio e duplamente ocupado, além de fazer $E_{d}=0$. Assim, primeiro devemos generalizar os resultados da Ref. [13].

Considere um modelo quadrático definido pelo Hamiltoniano $H=\sum \epsilon_{n} g_{n \mu}^{\dagger} g_{n \mu}$, onde cada nível $\epsilon_{n}$ pode estar vazio, unicamente ocupado ou duplamente ocupado. A função de partição é dada 
por:

$$
\begin{aligned}
Z & \equiv \operatorname{Tr}\left(e^{-\beta H}\right)=\sum_{\left\{r_{0}\right\}} \sum_{\left\{r_{1}\right\}} \ldots \sum_{\left\{r_{N}\right\}}\left\langle r_{0}, r_{1}, \ldots, r_{N}\left|e^{-\beta \sum_{n, \mu} \epsilon_{n} g_{n \mu}^{\dagger} g_{n \mu}}\right| r_{0}, r_{1}, \ldots, r_{N}\right\rangle \\
& =\sum_{\left\{r_{0}\right\}} \sum_{\left\{r_{1}\right\}} \ldots \sum_{\left\{r_{N}\right\}}\left\langle r_{0}\left|e^{-\sum_{\mu} \beta \epsilon_{0} g_{0 \mu}^{\dagger} g_{0 \mu}}\right| r_{0}\right\rangle\left\langle r_{1}\left|e^{-\sum_{\mu} \beta \epsilon_{1} g_{1 \mu}^{\dagger} g_{1 \mu}}\right| r_{1}\right\rangle \ldots\left\langle r_{N}\left|e^{-\sum_{\mu} \beta \epsilon_{n} g_{n \mu}^{\dagger} g_{n \mu}}\right| r_{N}\right\rangle \\
& =\prod_{n}^{N}\left\langle r_{n}\left|\sum_{\left\{r_{n}\right\}} e^{-\beta \epsilon_{n} g_{n \mu}^{\dagger} g_{n \mu}}\right| r_{n}\right\rangle=\prod_{n}\left(e^{-0 \beta \epsilon_{n}}+2 e^{-1 \beta \epsilon_{n}}+e^{-2 \beta \epsilon_{n}}\right) \\
& =\prod_{n}^{N}\left(1+e^{-\beta \epsilon_{n}}\right)^{2}
\end{aligned}
$$

onde $\left|r_{0}, r_{1}, \ldots, r_{N}\right\rangle$ é o estado de partículas independentes, sendo cada $\left\{r_{i}\right\}$ o conjunto dos possíveis números de ocupação do estado $i$.

A energia livre, $F=-k_{B} T \ln Z$, fica:

$$
F=-\frac{1}{\beta} \ln \left(\prod_{n}\left(1+e^{-\beta \epsilon_{n}}\right)^{2}\right)=-\frac{2}{\beta} \sum_{n} \ln \left(1+e^{-\beta \epsilon_{n}}\right)
$$

Levando para o contínuo,

$$
\begin{aligned}
F & =-\frac{2}{\beta} \int_{-\infty}^{\infty} \rho(\epsilon) \ln \left(1+e^{-\beta \epsilon}\right) d \epsilon=-\frac{2}{\beta} \int_{-\infty}^{\infty} \rho(\epsilon) \ln \left[e^{-\frac{\beta \epsilon}{2}}\left(e^{\frac{\beta \epsilon}{2}}+e^{-\frac{\beta \epsilon}{2}}\right)\right] d \epsilon \\
& =-\frac{2}{\beta} \int_{-\infty}^{\infty} \rho(\epsilon) \ln \left(e^{\frac{\beta \epsilon}{2}}+e^{-\frac{\beta \epsilon}{2}}\right) d \epsilon+\int_{-\infty}^{\infty} \rho(\epsilon) \epsilon d \epsilon,
\end{aligned}
$$

onde $\rho(\epsilon)=-\rho(-\epsilon)$ é a densidade de estados que, para esse modelo, é dada por [13]:

$$
\rho=\frac{\Delta / \pi}{\epsilon^{2}+\Delta^{2}}
$$

Então,

$$
F=-\frac{2}{\beta} \int_{-\infty}^{\infty} \rho(\epsilon) \ln \left(e^{\frac{\beta \epsilon}{2}}+e^{-\frac{\beta \epsilon}{2}}\right) d \epsilon
$$

Sendo o modelo quadrático, é relativamente fácil generalizar os resultados de Schotte e Schotte para incluir o nível de energia $E_{d} \pm B$ e também para estender o tratamento para o ensemble grão canônico, já que naquela referência os níveis eram unicamente ocupados. Para impurezas de spin $1 / 2, F$ passa 
a ser

$$
\begin{aligned}
F & =2 k_{B} T \operatorname{Re} \sum_{\mu= \pm}\left\{\ln \Gamma\left(1+\frac{\Delta+i\left(E_{d}+\mu B\right)}{\pi k_{B} T}\right)-\ln \Gamma\left(1+\frac{\Delta+i\left(E_{d}+\mu B\right)}{2 \pi k_{B} T}\right)\right\} \\
& +\frac{2 \Delta}{\pi}\left(1-\ln \frac{D}{\pi k_{B} T}\right)-k_{B} T \ln 4
\end{aligned}
$$

onde $\Gamma(x)$ é a função gama.

Então, o calor específico se escreve

$$
\frac{C}{k_{B}} \equiv-T \frac{\partial^{2} F}{\partial T^{2}}=2 \operatorname{Re} \sum_{\mu= \pm}\left[\alpha_{\mu}-\alpha_{\mu}^{2} \Psi^{\prime}\left(1 / 2+\alpha_{\mu}\right)\right]
$$

onde $\Psi^{\prime}$ é a função trigama (ver Apêndice A) e $\alpha_{\mu}=\frac{\Delta+i\left(E_{d}+\mu B\right)}{2 \pi k_{B} T}$, com a largura de nível sendo $\Delta=\pi V^{2}[13]$. A variação total da entropia do modelo, $\Delta S=\int_{0}^{\infty} \frac{C}{T} d T$, é $k_{B} \ln 4$, ao passo que para o modelo original de Schotte e Schotte é $k_{B} \ln 2$. Isto se deve ao fato de que eles consideram apenas duas possibilidades para a ocupação do nível enquanto consideramos quatro.

Para a suscetibilidade magnética temos

$$
\frac{k_{B} T \chi}{\left(g \mu_{B}\right)^{2}}=\frac{1}{8 \pi^{2}} \operatorname{Re} \sum_{\mu= \pm} \Psi^{\prime}\left(1 / 2+\alpha_{\mu}\right)
$$

enquanto que para a entropia temos

$$
\frac{S}{k_{B}}=4 \operatorname{Re} \sum_{\mu= \pm}\left\{\alpha_{\mu}\left[\Psi\left(1 / 2+\alpha_{\mu}\right)-1\right]+\ln \frac{\sqrt{2 \pi}}{\Gamma\left(1 / 2+\alpha_{\mu}\right)}\right\}
$$

sendo $\Psi$ a função digama [33] (ver Apêndice A).

A seguir, apresentaremos os resultados analíticos e os do GRN, comparativamente.

\subsection{Resultados numéricos para o MNR}

Para cada propriedade termodinâmica, é sempre computada a contribuição da impureza à mesma. Isto é, subtrai-se o valor de banda livre do valor encontrado para o sistema banda-impureza. 
Isso não só é um procedimento comum experimentalmente como também é prescrição seguida no GRN - o qual reescreve a banda de condução de forma a preservar o acoplamento da impureza com a banda via o operador $f_{0}$, como vimos no Cap. 2 - para a obtenção de quantidades termodinâmicas. Assim, o GRN determina propriedades de impurezas (acopladas com a banda) e não da banda em si. Para subtrair os valores de banda livre podemos utilizar a própria implementação do GRN mas com acoplamentos banda-impureza nulos, ou, o que é mais rápido computacionalmente, os desenvolvimentos do Apêndice B.

Por brevidade, não apresentaremos as curvas de magnetização e de suscetibilidade magnética. Comparamos as curvas de calor específico exatas de Schotte e Schotte com as obtidas através do GRN adaptado para campo magnético. Obtivemos ótimo acordo entre as duas abordagens. Apresentamos duas curvas para o calor específico para dois casos representativos do MNR. Na primeira curva, escolhemos $V=0.02 D$ e $E_{d}=0.01 D$ para três valores de campo (ver Fig. (3.2)).

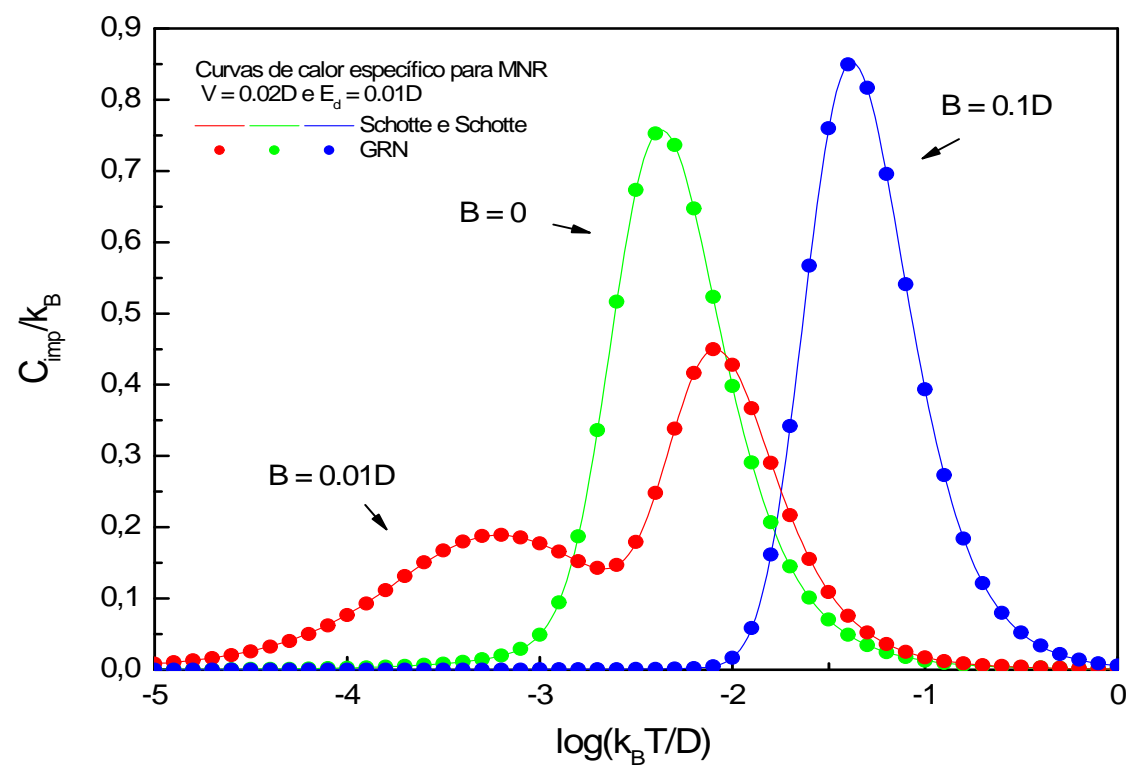

Figura 3.2: Curvas de calor específico para o MNR para três valores de campo, comparando-se o resultado analítico com o obtido através do GRN. 
Acompanhemos as Fig. (3.2), Fig. (3.3). Quando $B=0$ (curvas verdes), os níveis unicamente

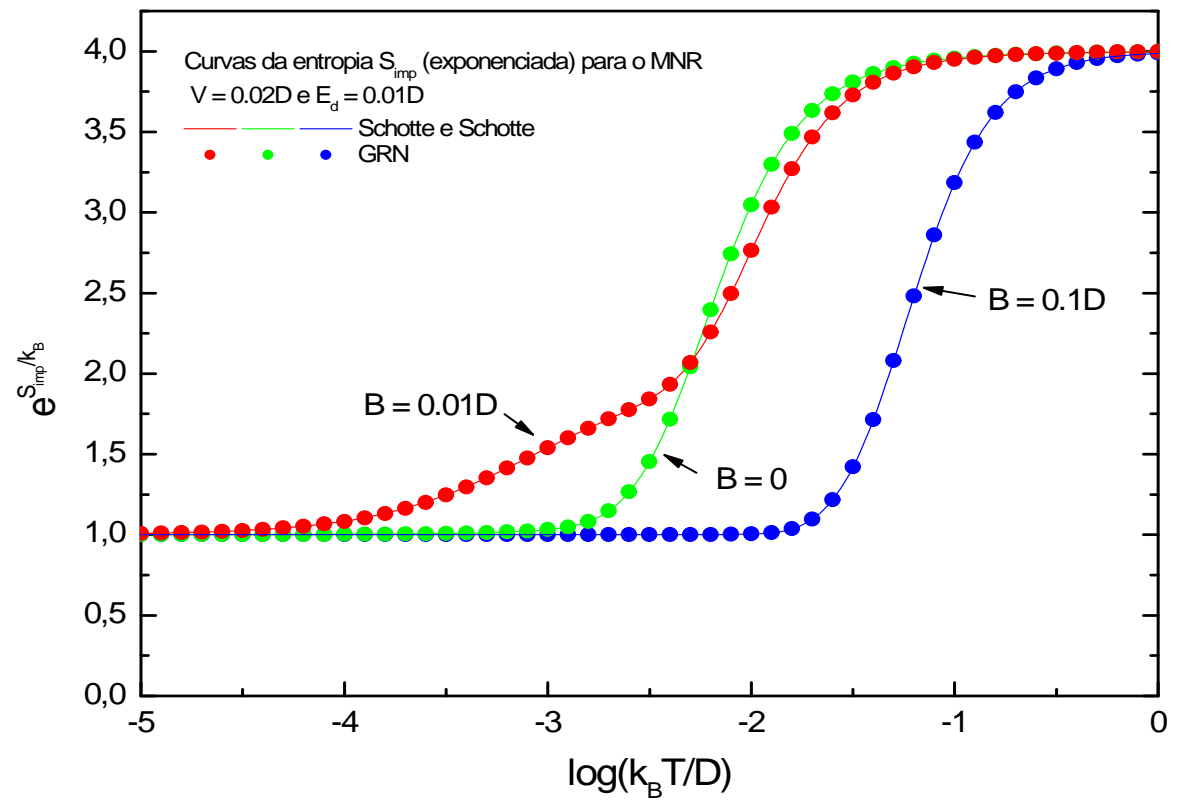

Figura 3.3: Curvas da entropia exponenciada, ou seja, do número de graus de liberdade correspondentes às curvas de calor específico da Fig. (3.2).

ocupados são degenerados entre si, e o nível desocupado é o de mais baixa energia. Em temperaturas altas, todos os quatro estados são praticamente degenerados $\left(e^{S_{i m p} / k_{B}}=4\right)$. À medida em que a temperatura abaixa, a probabilidade de transição diminui favorecendo o estado de mais baixa energia. No caso, o do nível desocupado. A presença do único pico no calor específico deve-se ao fato de que as transições permitidas exigem a mesma variação de energia $E_{d}$. Quando $B=0.01 D$ (curvas vermelhas), os níveis unicamente ocupados degeneram-se pelo efeito Zeeman com o nível ocupado e o duplamente ocupado. Como no caso anterior, a temperaturas elevadas todos são igualmente ocupáveis. Porém, há dois picos no calor específico. O primeiro (baixando-se a temperatura), refere-se à transição (com variação $2 E_{d}$ ) entre estes dois pares de níveis degenerados. Baixando-se ainda mais a temperatura, esta transição fica desfavorável chegando a um ponto em que a transição entre o nível vazio e o 
ocupado (alargado) com spin $\uparrow$ domina. Isto resulta no pico em $k_{B} T \sim \Delta$. Contudo, com $T \rightarrow 0$, as transições não são mais possíveis, resultando, como no caso anterior, apenas uma configuração permitida, favorecendo o nível desocupado.

Para $B=0.1 D$, o efeito Zeeman distancia suficientemente os níveis unicamente ocupados de tal forma que, a temperaturas elevadas, picos do calor específico referentes às transições permitidas se sobrepõem. Isso acontece porque a diferença de energia entre uma transição de um nível unicamente ocupado para o vazio e de unicamente ocupado para duplamente ocupado é pequena.

O MNR pode ser facilmente generalizado para o caso em que as hibrizações são dependentes da componente $z$ do spin. Com isso, ao invés de apenas $V$, teremos $V_{\uparrow}$ e $V_{\downarrow}$ :

$$
\begin{aligned}
H_{N} & =\Lambda^{\frac{N-1}{2}+z-1}\left\{\sum_{n=0}^{N-1}\left[t_{n}(B) f_{n+1, \uparrow}^{\dagger} f_{n, \uparrow}+\zeta_{n}(B) f_{n, \uparrow}^{\dagger} f_{n, \uparrow}\right]+\left(\widetilde{E}_{d}-\widetilde{B}\right) d_{\uparrow}^{\dagger} d_{\uparrow}+\widetilde{V}_{\uparrow}\left(f_{0, \uparrow}^{\dagger} d_{\uparrow}+\text { h.c. }\right)\right. \\
& \left.+\sum_{n=0}^{N-1}\left[t_{n}(B) f_{n+1, \downarrow}^{\dagger} f_{n, \downarrow}+\zeta_{n}(B) f_{n, \downarrow}^{\dagger} f_{n, \downarrow}\right]+\left(\widetilde{E}_{d}+\widetilde{B}\right) d_{\downarrow}^{\dagger} d_{\downarrow}+\widetilde{V}_{\downarrow}\left(f_{0, \downarrow}^{\dagger} d_{\downarrow}+\text { h.c. }\right)\right\}
\end{aligned}
$$

e nas fórmulas de Schotte e Schotte [13], basta substituir $\alpha_{\mu}=\frac{\Delta+i\left(E_{d}+\mu B\right)}{2 \pi k_{B} T}$ por $\alpha_{\mu}=\frac{\Delta_{\mu}+i\left(E_{d}+\mu B\right)}{2 \pi k_{B} T}$, onde $\pi V_{\uparrow}^{2}=\Delta_{+}$e $\pi V_{\downarrow}^{2}=\Delta_{-}$.

Acompanhemos as Figs. (3.4) e (3.5) :

As curvas em vermelho são referentes à hibridização nula para o nível de estado unicamente ocupado com spin para baixo $\left(V_{\downarrow}=0\right.$ e $\left.V_{\uparrow} \neq 0\right)$. A intensidade do campo gera a mesma estrutura que a das curvas vermelhas que discutimos anteriormente e a interpretação é similar. Com temperaturas elevadas, todas as transições permitidas conseguem ocorrer e o primeiro pico (da direita à esquerda) refere-se a energia $2 E_{d}$. Como os estados mais energéticos têm hibridização nula, à medida que a temperatura abaixa, somente as transições virtuais entre o nível desocupado e o unicamente ocupado com spin para cima ocorrem, gerando o segundo pico. Mas, no limite de temperatura nula, nem estas acontecem, fazendo com que, de novo, haja apenas uma configuração no final.

Quando a hibridização do estado unicamente ocupado com spin para cima é nula $\left(V_{\uparrow}=0\right)$, 


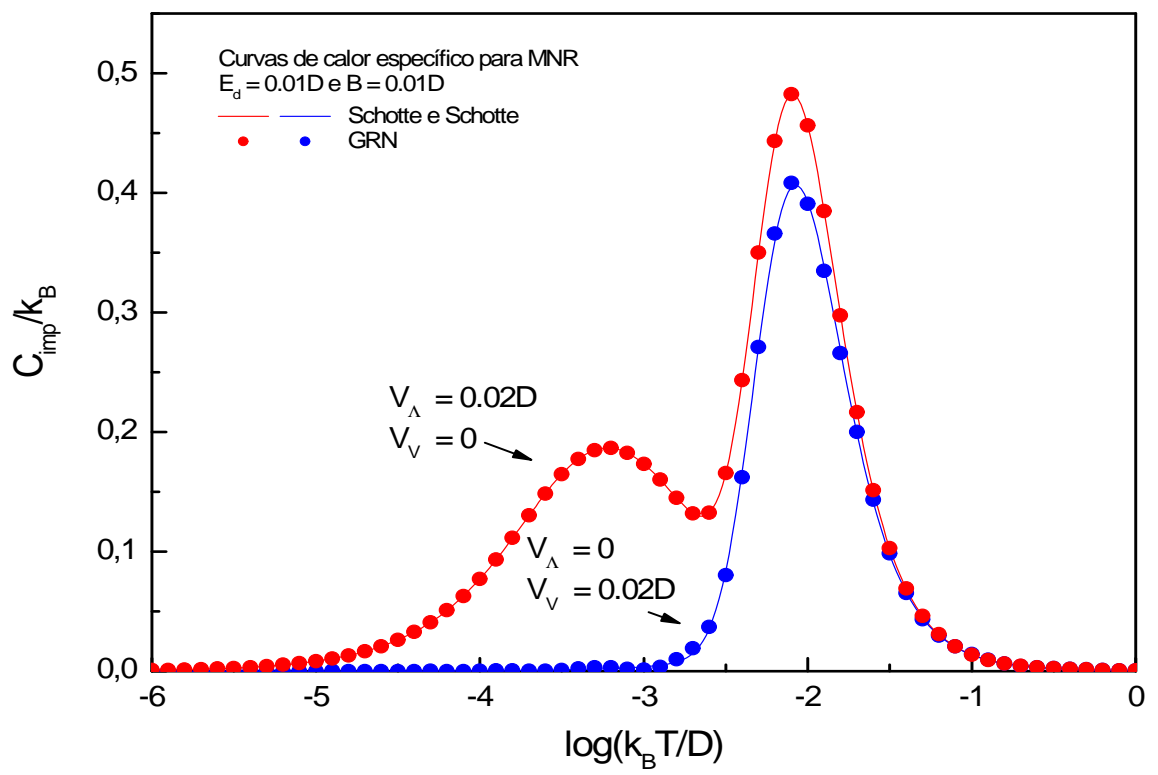

Figura 3.4: Curvas de calor específico para o caso em que a hibridização é dependente do spin.

teremos apenas um pico que é devido às mesmas razões do caso que acabamos de discutir. Porém, à medida em que a temperatura abaixa, já não é possível, como acontecera no outro caso, que haja as transições virtuais. Estas só podem ocorrer com variação de energia quando há largura no nível.

Estendendo a aplicação do que acabamos de estudar para o MNR, implementamos o mesmo procedimento para o Modelo de Falicov-Kimball do qual tratamos a seguir. 


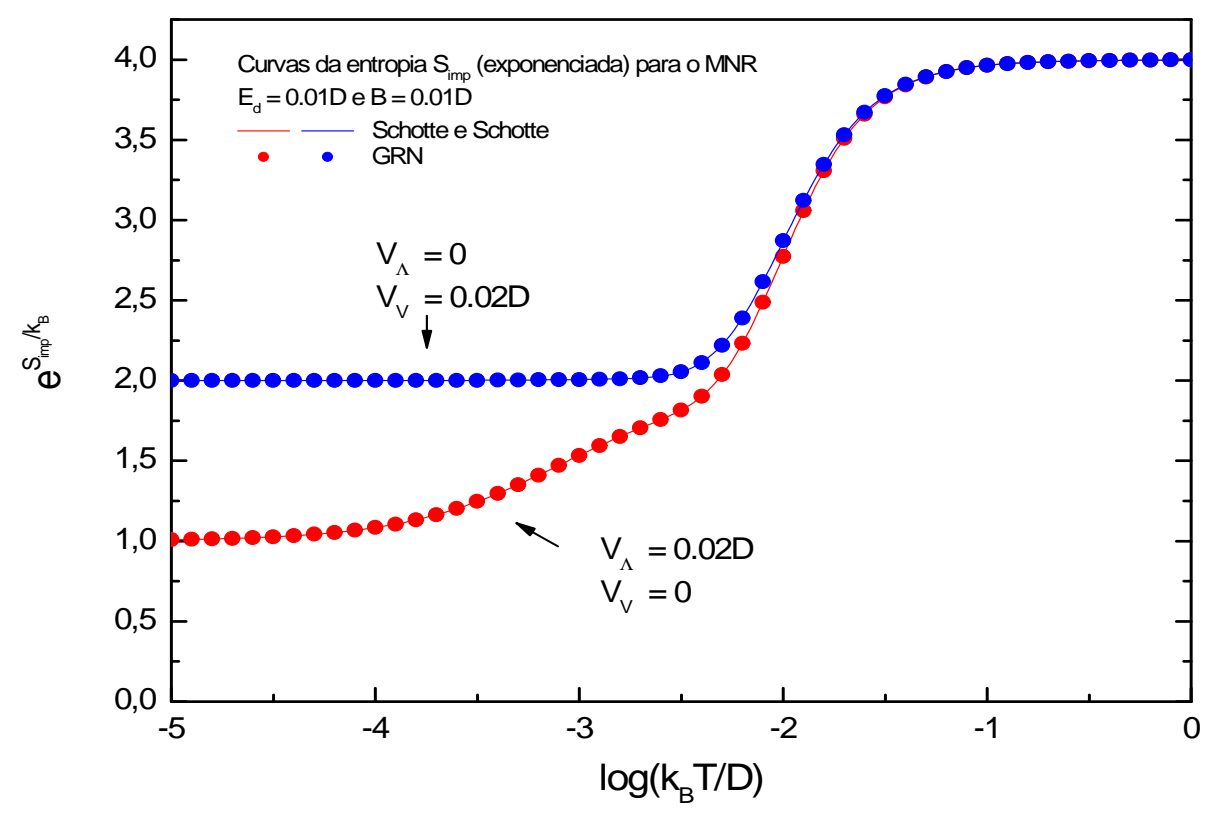

Figura 3.5: Curvas da entropia exponenciada, ou seja, dos graus de liberdade correspondentes às curvas de calor específico da Fig. (3.4). 


\section{Capítulo 4}

\section{Modelo de Falicov-Kimball (MFK)}

Proposto em 1969 [9, 14], ainda atualmente tem despertado grande interesse [15-21]. Este modelo descreve a interação eletrostática de impurezas hibridizadas com a banda de condução de um metal. Esse modelo já foi usado para estudar a transição metal-semicondutor em óxidos [9, 14] e recentemente foi aplicado no estudo da transição metal-isolante [17]. Em geral, sua aplicação, no entanto, se dá ou em uma dimensão $[18,19]$ ou em infinitas dimensões, [15, 20], ou ainda sem levar em conta a hibridização entre os níveis localizados e os de condução [19, 21].

Neste Capítulo estudamos esse modelo com duas impurezas localizadas interagindo com um banho fermiônico. Embora seja um caso particular do modelo com uma rede de impurezas, devido à hibridização com a banda, o modelo é não trivial e exige tratamento especial para se diagonalizar seu Hamiltoniano. Por isso, iremos utilizar o GRN e como ainda queremos tratar uma banda não simétrica em relação ao nível de Fermi, será imprescindível o formalismo do capítulo anterior.

Como o modelo não tem spin, ao invés de calcular a suscetibilidade magnética, que mede a flutuação dos spins, iremos calcular uma função resposta à flutuação de carga. Para isso, vamos acoplar as cargas do modelo a um campo externo (campo de carga), que por analogia ao campo magnético estudado no Capítulo anterior, também denotaremos por B. Assim, do espectro computado via GRN 
determinaremos o calor específico e a suscetibilidade de carga do modelo ambos a campo não nulo.

\subsection{O Modelo}

O modelo de Falikov-Kimball na presença de um campo de carga $B$ é definido pelo Hamiltoniano

$$
\begin{aligned}
H & =\sum_{\vec{k}} \epsilon_{k} c_{\vec{k}}^{\dagger} c_{\vec{k}}-B \sum_{\vec{k}} c_{\vec{k}}^{\dagger} c_{\vec{k}}+E_{d}\left(d_{1}^{\dagger} d_{1}+d_{2}^{\dagger} d_{2}\right)-B\left(d_{1}^{\dagger} d_{1}+d_{2}^{\dagger} d_{2}\right) \\
& +G\left[\Psi^{\dagger}(\vec{R} / 2) \Psi(\vec{R} / 2) d_{1}^{\dagger} d_{1}+\Psi^{\dagger}(-\vec{R} / 2) \Psi(-\vec{R} / 2) d_{2}^{\dagger} d_{2}\right] \\
& +V\left[\Psi^{\dagger}(\vec{R} / 2) d_{1}+\Psi^{\dagger}(-\vec{R} / 2) d_{2}+\text { H.c. }\right]
\end{aligned}
$$

onde o primeiro termo descreve uma banda de condução com relação de dispersão $\epsilon_{k}$, medida do nível de Fermi. O segundo termo corresponde à energia dos elétrons de condução na presença do campo $B$. Os operadores $d_{1}^{\dagger}$ e $d_{2}^{\dagger}$ criam estados fermiônicos centrados respectivamente em $\vec{R} / 2$ e $-\vec{R} / 2$; eles são degenerados, com energia $E_{d}$, e submetidos também ao campo $B$. O operador de

campo $\Psi(\vec{r})=\sum_{\vec{k}} e^{i \vec{k} \cdot \vec{r}} c_{\vec{k}}$ destrói um elétron de condução na posição $\vec{r}$. Os termos proporcionais a $G$ descrevem o espalhamento Coulombiano entre as cargas localizadas em $\pm \vec{R} / 2$ com aquelas da banda. Os termos proporcionais a $V$ descrevem a hibridização entre os orbitais de condução e os localizados. Paridade é conservada já que o Hamiltoniano é invariante sob a troca $\vec{R} \leftrightarrow-\vec{R}$ e $d_{1} \leftrightarrow d_{2}$. No entanto, não temos simetria partícula-buraco devido aos termos de espalhamento e de campo externo. Spins não são incluídos no modelo uma vez que não desempenham papel na dinâmica (a menos que uma correlação local fosse incluída, como no modelo de Anderson).

A assimetria da banda aparece nesse modelo quando pensamos no primeiro e segundo termos de $H$ juntos, resultando no termo

$$
\sum_{\vec{k}}\left(\epsilon_{k}-B\right) c_{\vec{k}}^{\dagger} c_{\vec{k}}
$$


Pela experiência dos Capítulos anteriores, é esse termo combinado que devemos discretizar logaritmicamente, e não o termo cinético em separado.

Para maior clareza, primeiro vamos desenvolver os passos do GRN para uma banda simétrica $(B=0)$ e no final introduzir $B$ baseado na experiência com o MNR. A vantagem é que a implementação do GRN para bandas simétricas já é conhecida para esse modelo de duas impurezas [22, 23, 24, 25, 26].

\subsection{Grupo de Renormalização Numérico}

Não iremos detalhar todo o procedimento do GRN, mas sim fornecer apenas aqueles necessários aos nossos propósitos. Seguiremos de perto as Ref. [22, 23, 24, 25, 26]. O que o GRN faz é transformar o Hamiltoniano acima em uma seqüência de Hamiltonianos efetivos $H_{N}$ que são diagonalizados recursivamente. Dos espectros de $H_{N}$ são computadas funções termodinâmicas para um certo intervalo de temperatura.

Primeiramente, adotamos uma relação de dispersão isotrópica, isto é, dependente apenas do módulo de $\vec{k}: \epsilon_{\vec{k}}=\epsilon_{k}$. Assim, é oportuno reescever os operadores de campo em termos de novos operadores dependentes apenas da energia $\epsilon \equiv \epsilon_{k}$ :

$$
\Psi\left(\frac{\vec{R}}{2}\right)=\int d^{3} k e^{i \vec{k} \cdot \frac{\vec{R}}{2}} c_{\vec{k}}=\int d^{3} k \int d \epsilon \delta\left(\epsilon-\epsilon_{k}\right) e^{i \vec{k} \cdot \frac{\vec{R}}{2}} c_{\vec{k}} \equiv \int_{-D}^{D} d \epsilon \sqrt{\rho(\epsilon)} c_{1 \epsilon},
$$

onde a última igualdade define o operador

$$
c_{1, \epsilon}=\frac{1}{\sqrt{\rho}} \int d^{3} k e^{i \vec{k} \cdot \vec{R} / 2} \delta\left(\epsilon-\epsilon_{k}\right) c_{\vec{k}}
$$

com $\rho(\epsilon)=\int d^{3} k \delta\left(\epsilon-\epsilon_{k}\right)$, a densidade de estados. Analogamente,

$$
\Psi\left(\frac{-\vec{R}}{2}\right)=\int d^{3} k e^{-i \vec{k} \cdot \vec{R}} \frac{\vec{R}}{\vec{k}} \equiv \int_{-D}^{D} d \epsilon \sqrt{\rho(\epsilon)} c_{2 \epsilon}
$$

define o operador

$$
c_{2, \epsilon}=\frac{1}{\sqrt{\rho}} \int d^{3} k e^{-\vec{k} \cdot \vec{R} / 2} \delta\left(\epsilon-\epsilon_{k}\right) c_{\vec{k}}
$$


Os operadores $c_{i, \epsilon}$ não são ortonormais: $\left\{c_{1 \epsilon}, c_{2 \epsilon^{\prime}}^{\dagger}\right\}=\delta\left(\epsilon-\epsilon^{\prime}\right)(\sin (k(\epsilon) R)) /(k(\epsilon) R)$. Então, definimos novos operadores $s_{\epsilon \pm}$ através de

$$
s_{\epsilon+}=\frac{c_{1 \epsilon}+c_{2 \epsilon}}{2 A_{+}} \quad \text { e } \quad s_{\epsilon-}=\frac{c_{1 \epsilon}-c_{2 \epsilon}}{2 A_{-}}
$$

com

$$
A_{ \pm}^{2}=(1 \pm S) / 2, \quad \text { sendo } \quad S=\frac{\sin (k R)}{k R}
$$

Esses operadores são ortonormais e obedecem à relação $\left\{s_{\epsilon \pm}, s_{\epsilon^{\prime} \pm}^{\dagger}\right\}=\delta\left(\epsilon-\epsilon^{\prime}\right)$, têm paridade definida, e em termos deles temos que

$$
\begin{aligned}
H & =\sum_{k}\left(\epsilon_{k}-B\right) c_{k}^{\dagger} c_{k}+\left(E_{d}-B\right)\left(d_{+}^{\dagger} d_{+}+d_{-}^{\dagger} d_{-}\right) \\
& +2 G\left[\left(A_{+}^{2} f_{0,+}^{\dagger} f_{0,+}+A_{-}^{2} f_{0,-}^{\dagger} f_{0,-}\right)\left(d_{+}^{\dagger} d_{+}+d_{-}^{\dagger} d_{-}\right)\right. \\
& \left.+2 A_{+} A_{-}\left(f_{0,+}^{\dagger} f_{0,-}+f_{0,-}^{\dagger} f_{0,+}\right)\left(d_{+}^{\dagger} d_{-}+d_{-}^{\dagger} d_{+}\right)\right] \\
& \left.+2 V\left[A_{+} f_{0,+}^{\dagger} d_{+}+A_{-} f_{0,-}^{\dagger} d_{-}+\text {H.c. }\right]\right\}
\end{aligned}
$$

sendo que os seguintes operadores com paridade definida foram convenientemente introduzidos:

$$
d_{ \pm}=\left(d_{1} \pm d_{2}\right) / \sqrt{2} \quad \text { e } \quad f_{0, \pm}=\frac{1}{\sqrt{2 D}} \int_{-D}^{D} d \epsilon s_{\epsilon \pm}
$$

Fica fácil notar que $H$ conserva paridade, pois todos os termos são simétricos pela troca dos índices $+\leftrightarrow-$. Também usamos que, como a largura de banda $D$ é da ordem de elétron-volt, as excitações responsáveis pela termodinâmica do modelo sendo da ordem de $1 \mathrm{meV}$, são portanto muito menores que $D$, e então podemos substituir $\rho(\epsilon)$ e $A_{ \pm}(k)$ pelos seus valores no nível de Fermi: ${ }^{1} \rho(\epsilon=0)=1 / D$ e $A_{ \pm}\left(k=k_{F}\right) \equiv A_{ \pm}$

Neste ponto entra em cena a discretização logarítmica da banda. Para cada paridade, procedemos da mesma maneira que fizemos para chegar à Eq. (2.9) partindo da banda de condução Eq.

\footnotetext{
${ }^{1} \mathrm{O}$ valor $\rho(0)=1 / D$ é consistente com a normalização necessária dos operadores de campo $\Psi(\vec{r})$.
} 
(2.3). Chegamos, então, ao seguinte Hamiltoniano transformado²

$$
\begin{aligned}
H_{N} & =\Lambda^{\frac{N-1}{2}}\left\{\sum_{n=0}^{N-1} t_{n}(z)\left(f_{n,+}^{\dagger} f_{n+1,+}+f_{n,-}^{\dagger} f_{n+1,-}+h . c .\right)+\sum_{n=0}^{N} \zeta_{n}(z)\left(f_{n+}^{\dagger} f_{n+}+f_{n-}^{\dagger} f_{n-}\right)\right. \\
& +\left(\bar{E}_{d}-\bar{B}\right)\left(d_{+}^{\dagger} d_{+}+d_{-}^{\dagger} d_{-}\right) \\
& +2 \bar{G}\left[\left(A_{+}^{2} f_{0,+}^{\dagger} f_{0,+}+A_{-}^{2} f_{0,-}^{\dagger} f_{0,-}\right)\left(d_{+}^{\dagger} d_{+}+d_{-}^{\dagger} d_{-}\right)\right. \\
& \left.+2 A_{+} A_{-}\left(f_{0,+}^{\dagger} f_{0,-}+f_{0,-}^{\dagger} f_{0,+}\right)\left(d_{+}^{\dagger} d_{-}+d_{-}^{\dagger} d_{+}\right)\right] \\
& \left.+2 \bar{V}\left[A_{+} f_{0,+}^{\dagger} d_{+}+A_{-} f_{0,-}^{\dagger} d_{-}+H_{. c .}\right]\right\} .
\end{aligned}
$$

Aqui introduzimos a notação $\bar{G}=p G, \bar{E}_{d}=p E_{d}$ e $\bar{B}=p B$, sendo $p \equiv 2 /\left(1+\Lambda^{-1}\right)$. O prefator $\Lambda^{(N-1) / 2}$ na Eq. (4.11) torna, convenientemente, os menores elementos de matriz de $H_{N}$ próximos à unidade [2]. O Hamiltoniano inicial $H$ é recuperado fazendo-se

$$
H=\frac{1+\Lambda^{-1}}{2} \lim _{N \rightarrow \infty} \Lambda^{-\frac{N-1}{2}-z+1} H_{N}
$$

No trabalho original de Wilson [2] a discretização é feita com apenas um parâmetro, $\Lambda>1$, tal que qualquer energia $\epsilon$ está no intervalo $\Lambda^{-n-1-z}<|\epsilon / D|<\Lambda^{-n-z}$ para algum inteiro $n$. O uso do parâmetro adicional $z>0[8]$ tal que $\Lambda^{-n-1-z}<|\epsilon / D|<\Lambda^{-n-z}$ é muito conveniente ao se eliminar os efeitos espúrios da discretização da banda que aparecem nas propriedades físicas, isto é, quando se deseja retornar ao contínuo inicial [22, 27, 23, 28]. Esses efeitos aparecem como oscilações quando se expressa uma propriedade termodinâmica em função da temperatura. Para $n>>1, t_{n}(z)$ vai assintoticamente como $\Lambda^{1-z-n / 2} D$, enquanto $\zeta_{n} \approx \Lambda^{-n}$, o que assegura uma convergência rápida da série em $H_{N}$ e assim permite que a trunquemos em um dado $n=N$, mantendo os estados até $f_{N, \pm}$ em $H_{N}$.

O que torna o modelo de FK mais complexo que o MNR é ter operadores quárticos, ou seja, não ter um espectro de partículas independentes com o qual poderíamos construir os estados de

\footnotetext{
${ }^{2}$ Embora estejamos mantendo a notação $f_{0 \pm}$, com a assimetria esse operador não é mais dado pela Eq. (4.10); ele será dado, para cada canal de paridade, pelo operador $f_{0}$ definido na Eq. (2.11), onde os operadores $a, b, a_{m}$ e $b_{m}$ são aqui recolocados por $a_{ \pm}, b_{ \pm}, a_{m \pm}$ e $b_{m \pm}$ definidos na Eq. (2.7) com $c_{\epsilon}$ trocado por $s_{\epsilon \pm}$.
} 
muitos corpos necessários à termodinâmica do modelo. Sendo assim, sua diagonalização não é trivial e no contexto do GRN isso é implementado primeiramente observando que reescrevendo a Eq. (4.11) para $H_{N+1}$ implica na seguinte relação de recorrência, conhecida como a transformação do Grupo de Renormalização:

$$
\mathcal{T}\left[H_{N}\right] \equiv H_{N+1}=\sqrt{\Lambda} H_{N}+\Lambda^{N / 2} \sum_{p= \pm}\left[t_{N}(z)\left(f_{N, p}^{\dagger} f_{N+1, p}+h . c .\right)+\zeta_{N+1}(z) f_{N+1, p}^{\dagger} f_{N+1, p}\right]
$$

que permite que diagonalizemos $H_{N+1}$ conhecido o espectro de $H_{N}$. Então, começamos diagonalizando $H_{N=0}$ analiticamente. Com seus autovetores construímos a base de estados para montar $H_{N=1}$. Dos autovetores de $H_{N=1}$ montamos a base para $H_{N=2}$, e assim sucessivamente até que a escala de energia de interesse, tipicamente da ordem de $\Lambda^{-N / 2}$, seja alcançada.

Como já discutido nas Ref. [27, 28], médias termodinâmicas $A(T, \Lambda, z)$, computadas com z e $\Lambda$, convergem para o limite contínuo $A(T)$ muito rapidamente com $\Lambda$ tendendo à unidade:

$$
A(T, \Lambda, z)=A(T)+f_{A}(T, \Lambda, z) e^{-\pi^{2} / \ln \Lambda}
$$

onde a função $f_{A}$ depende da propriedade física, mas é periódica, com período $\ln T / \ln \Lambda$ na variável $\Lambda$. Também é composta de uma série com termos oscilatórios em $z$, sendo o termo predominante da série aquele com período unitário em $z$. Portanto, média de $A(T, \Lambda, z)$ num intervalo unitário de $z$ remove eficientemente na prática as oscilações expúrias e assim recupera o limite contínuo $A(T)$.[27]

Nós escolhemos trabalhar com $\Lambda=4$ e para todo o espaço de parâmetros do nosso modelo foram suficientes dois valores de $z$ nessa média, isto é, o limite contínuo foi calculado pela média

$$
A(T)=\frac{A(T, \Lambda=4, z=0.75)+A(T, \Lambda=4, z=1.25)}{2}
$$

Valores outros de $\Lambda$ ou mesmo média com quatro valores de $z$ resultaram em praticamente as mesmas curvas. 


\subsection{Resultados}

Uma vez determinado o espectro de energias $E_{N}$ de $H_{N}$, o calor específico é computado via a fórmula

$$
C_{N}=\frac{\partial \bar{E}_{N}}{\partial T} \quad \text { com } \quad \bar{E}_{N}=\left\langle H_{N}\right\rangle
$$

resultando em

$$
\frac{C_{N}}{k_{B}}=\beta^{2}\left(<E_{N}^{2}>-<E_{N}>^{2}\right)
$$

com $<. .>$ denotando média termodinâmica; aqui $\beta=1 / k_{B} T$. A contribuição das impurezas ao calor específico é obtida da diferença $C_{i m p}=C_{N}-C_{b c}$, isto é, subtrai-se do calor específico calculado para band + impureza, $C_{N}$, aquele determinado somente com a banda livre, $C_{b c}$.

Computamos também a suscetibilidade de carga, definida por

$$
\chi_{N}=\frac{\partial \bar{P}_{N}}{\partial B}, \quad \text { com } \quad \bar{P}_{N}=\left\langle Q_{N}\right\rangle
$$

onde $Q_{N}$ é o operador carga total no $N$-ésimo passo do GRN:

$$
Q_{N}=d_{1}^{\dagger} d_{1}+d_{2}^{\dagger} d_{2}+\sum_{n, p= \pm}^{N} f_{n, p}^{\dagger} f_{n, p}
$$

Disso resulta que

$$
T \chi_{N}=<Q_{N}^{2}>-<Q_{N}>^{2}
$$

Essas médias são calculadas diretamente do espectro de energias (envolvem elementos de matriz simples uma vez que a carga é um número quântico do problema). A contribuição dos estados localizados, $T \chi_{i m p}$, é calculada subtraindo-se o valor de banda livre, isto é, $T \chi_{i m p}=T \chi_{N}-T \chi_{b c}$.

Observe que a suscetibilidade magnética definida para os modelos com spin tem uma expressão análoga a essa, com $Q_{N}$ substituído por $g \mu_{B} S_{z, N}$.

Abaixo apresentamos os resultados numéricos divididos em quatro subseções. Primeiro consideramos um caso trivial, mas instrutivo, em que $G=V=0$, mas com $E_{d}$ e $B$ não nulos. 


\subsection{1 $\mathrm{G}=\mathrm{V}=0$}

A Fig. (4.1) ilustra este caso para $E_{d}=0.001 D, k_{F} R$ qualquer e $B=0.01 D$.

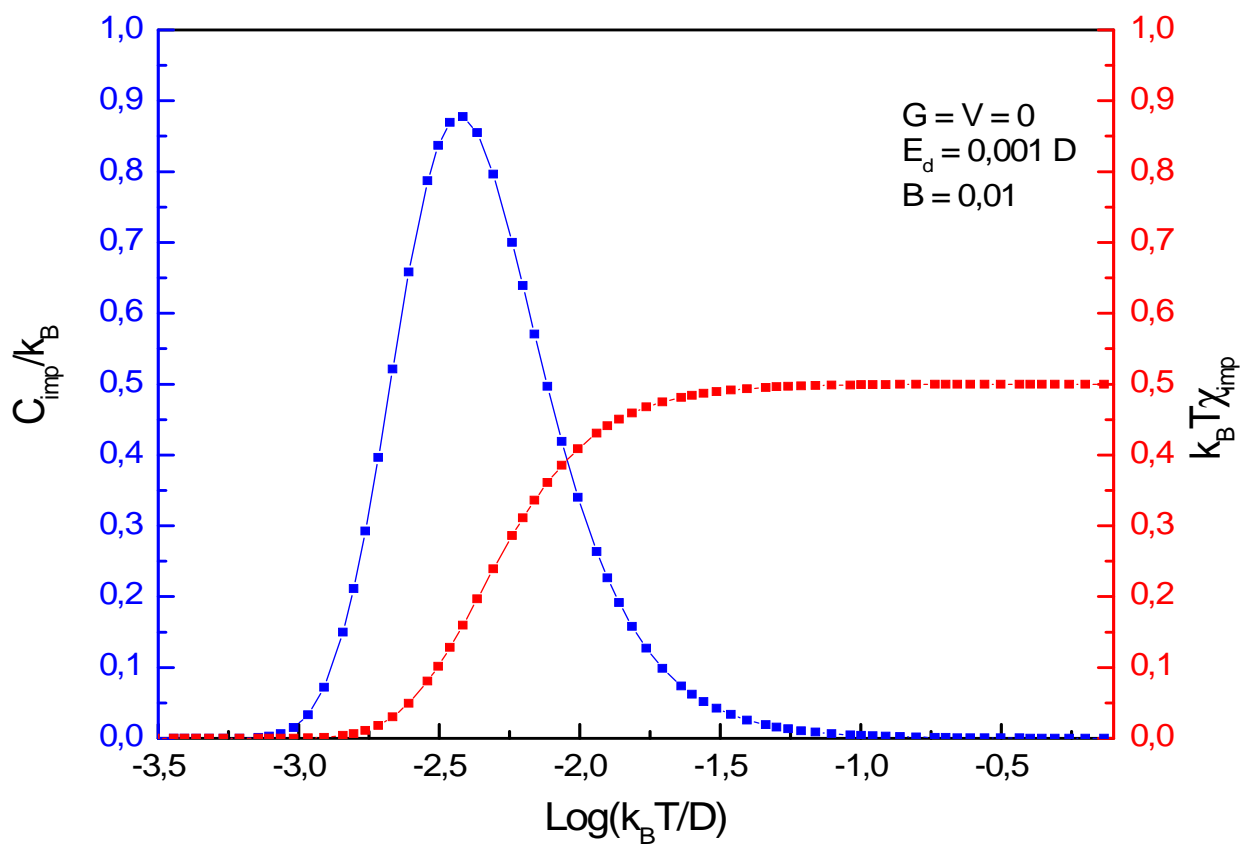

Figura 4.1: Curvas para suscetibilidade de carga e calor específico do MFK num caso em que há solução analítica. A temperatura de pico do calor específico (curva azul) $T_{\text {pico }}$ é dada por $k_{B} T_{\text {pico }}=$ $0,416778 E_{d}^{*}$, onde $E_{d}^{*}=\left|E_{d}-B\right|$.

Os pontos são nossos resultados obtidos através do GRN enquanto as curvas contínuas são obtidas considerando-se um sistema de três níveis advindos do Hamiltoniano de impureza livre dado por

$$
H=\left(E_{d}-B\right)\left(d_{1}^{\dagger} d_{1}+d_{2}^{\dagger} d_{2}\right)
$$

É imediato determinar $C_{i m p}$ com esse Hamiltoniano:

$$
\frac{C_{i m p}}{k_{B}}=2\left(\beta E_{d}^{*}\right)^{2} \frac{e^{-\beta E_{d}^{*}}}{\left(1+e^{-\beta E_{d}^{*}}\right)^{2}}
$$


também é imediato obter $k_{B} T \chi$ :

$$
k_{B} T \chi=2 \frac{e^{-\beta E_{d}^{*}}+2 e^{-2 \beta E_{d}^{*}}+e^{-\beta 3 E_{d}^{*}}}{\left(1+e^{-\beta E_{d}^{*}}\right)^{4}} .
$$

Como se vê, nesse regime as funções termodinâmicas dependem universalmente da variável $E_{d}^{*}=$ $E_{d}-B$; curvas para diferentes $E_{d}$ ou $B$ são simples translações dessa curva no eixo das temperaturas.

A física envolvida nessas curvas é relativamente simples. Em altas temperaturas, ou seja, $k_{B} T>>\left|E_{d}-B\right|$, as quatro configurações: vazio (energia zero), uma carga em $d_{+}$ou $d_{-}$(energia $E_{d}-B$ ), ou duas cargas (energia $2 E_{d}-2 B$ ) são praticamente degeneradas e assim o calor específico vai a zero (a energia média vai a uma constante). Já $k_{B} T \chi_{i m p}$ tem seu valor máximo, 1/2, uma vez que praticamente não há custo energético para um elétron saltar de uma configuração de carga à outra . Para temperaturas muito baixas, isto é, $k_{B} T<<\left|E_{d}-B\right|$, apenas uma configuração domina a termodinâmica; pode ser a configuração vazia, para $E_{d}-B>0$, ou com duas partículas, se $E_{d}-B<0$, estando as demais configurações em muito alta energia (portanto, com fatores de Boltzmann desprezíveis). Assim, o calor específico vai a zero novamente (pois a energia média vai a uma constante) e também vai a zero a suscetibilidade de carga uma vez que não há mais flutuação de carga (o custo para o elétron saltar de uma configuração para outra é $\left|E_{d}-B\right|$, neste caso, $>>k_{B} T$ ).

A temperatura do pico do calor específico e o ponto de inflexão de $k_{B} T \chi_{i m p}$ podem ser determinados das equações acima. Eles coincidem e valem aproximadamente

$$
k_{B} T_{\text {pico }}=0.416778 E_{d}^{*}
$$

Essa equação servirá para definir o valor efetivo $E_{d}^{*}$ mesmo quando $G$ ou $V$ não forem nulos, através da determinação numérica de $T_{p i c o}$.

\subsection{2 $\mathrm{G} \neq 0$ e $\mathrm{V}=0$}

Para $V=0, E_{d}=0.001 D, k_{F} R=1$ e $G=0.01 D$ a Fig. (4.2) ilustra o calor específico para diversos valores de $B$. Todos os pontos se ajustam à função dada pela Eq.(4.22) com um valor de $E_{d}^{*}$ 


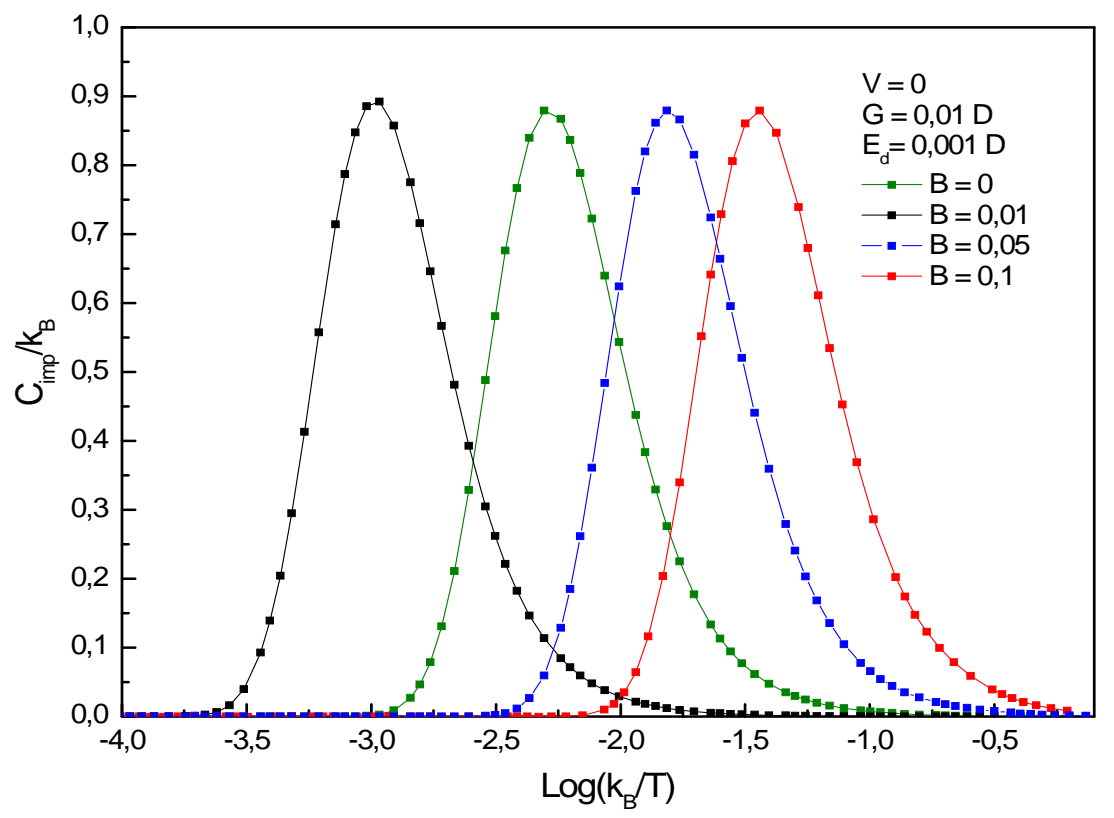

Figura 4.2: Curvas de calor específico do MFK para diversos valores de $B$ para $G \neq 0$ e $V=0$.

convenientemente escolhido, que depende de $G, k_{F} R$, além de $E_{d}$ e $B$. Essa escolha é feita usando-se o valor da temperatura de pico e a Eq. (4.24). Graficamente, observamos que existe um particular valor de $B$, chamemos de $B_{\text {pico, }}$, tal que o aumento da intensidade do campo tende a deslocar a curva de calor específico para a direita/esquerda se $B<B_{\text {pico }} / B>B_{\text {pico }}$. A Fig. (4.3) ilustra a dependência de $T_{\text {pico }} \operatorname{com} B$, para $E_{d}=0.001 D, k_{F} R=1$, e dois valores de $G$. Como se vê, o valor de $T_{\text {pico }}$ é muito pequeno no ponto de retorno, e o valor de $B$ que resulta nesse ponto de retorno depende de $G$. Essa dependência é natural se olharmos para o Hamiltoniano e percebemos que $B$ entra no termo $\zeta_{n}(B)$, e o maior destes termos é o $\zeta_{0}$ que é o coeficiente do operador $f_{0}^{\dagger} f_{0}$. Mas $G$ também aparece no coeficiente de $f_{0}^{\dagger} f_{0}$ dos termos de espalhamento em $H_{N}$. Assim, $G$ e $B$ competem para definir o ponto de retorno do calor específico.

A Fig. (4.4), para a suscetibilidade de carga, mostra que ela segue o mesmo padrão de 


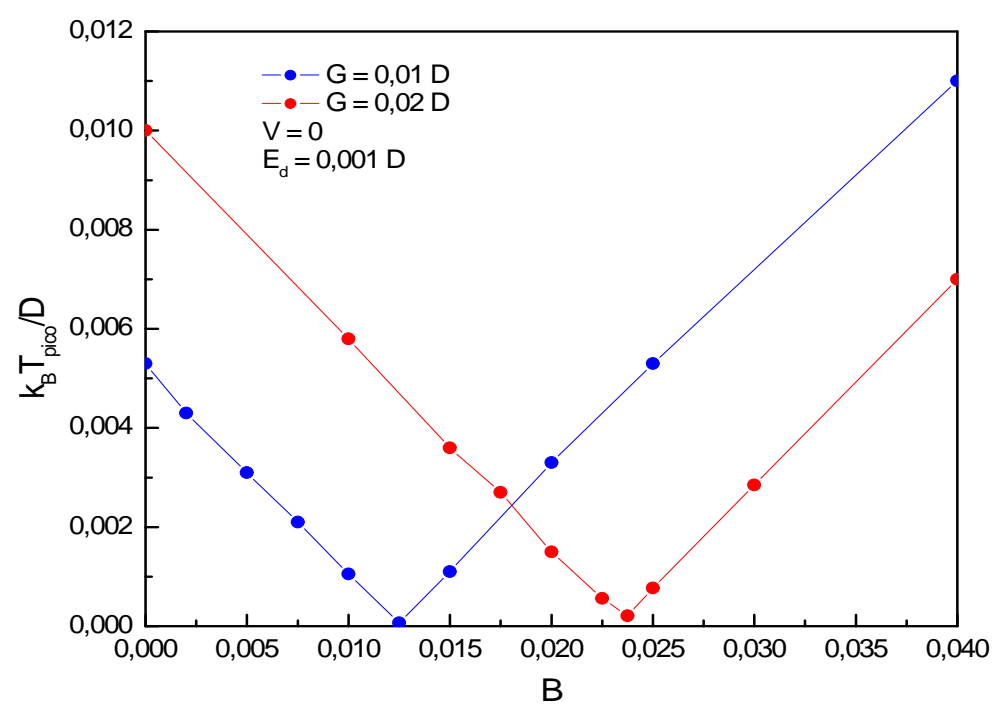

Figura 4.3: Decrição gráfica da variação da posição do pico das curvas do calor específico do MFK em função do campo $B$, sendo $V=0$ e $E_{d}=0,001 D$, para dois valores do termo de espalhamento Coulombiano: $G=0,01 D$ e $G=0,02 D$. Notemos que os valores de $B$ a partir dos quais as inclinações das retas mudam de sinal é ligeiramente maior que os $G$ 's correspondentes.

comportamento do calor específico.

\subsection{3 $\mathrm{G}=0, \mathrm{~V} \neq 0$}

Como vemos pela Fig. (4.5), para esses parâmetros as curvas não mantém o formato dado pelas Eq. (4.22) e (4.23). O alargamento dos níveis ocupados da impureza, devido à hibridização, faz com que a curva de calor específico se alargue e, consequentemente, se abaixe; isso para manter sua área constante e igual à variação total da entropia, que vale $S(T \approx D)-S(T=0)=k_{B} \ln 4$, visto que em alta temperatura temos quatro níveis, enquanto, para baixas temperaturas, apenas um nível localizado efetivamente participa da termodinâmica (lembre-se que $S(T)=k_{B} \ln W$, onde $W$ é o número de configurações do sistema que efetivamente participam da termodinâmica).

Conforme o campo $B$ aumenta, os níveis ocupados da impureza ficam com energia efetiva $E_{d}^{*}$ cada vez mais negativa e, portanto, cada vez menos será favorável uma troca de carga com a 


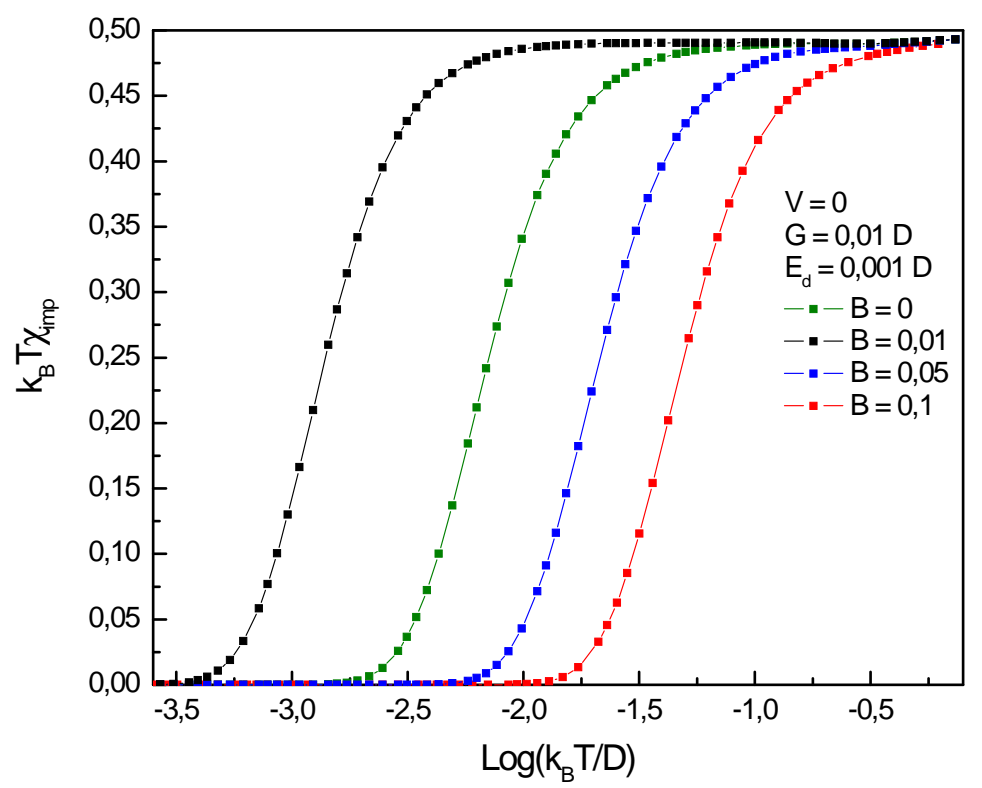

Figura 4.4: Curvas da suscetibilidade de carga do MFK para diversos valores de $B$ para $V=0$, $G=0,01 D$ e $E_{d}=0,001 D$.

banda. Assim, as curvas tendem àquela dada pelo Hamiltoniano de impureza livre, Eq. (4.21), com $E_{d}^{*}$ se aproximando de $\left|E_{d}-B\right|$ para $B>>E_{d}$. A seguir, temos as correspondentes curvas para a suscetibilidade de carga na Fig. (4.6).

Quanto ao comportamento de $C_{i m p} / k_{B}$ para $T \rightarrow 0$, vemos uma diferença marcante para o mesmo regime de $C_{i m p} / k_{B}$ do item anterior. Enquanto antes tínhamos um crescimento exponencial para $C_{i m p}(T) / k_{B}$, refletindo a presença de um gap de energia (ver Eq. (4.22) para $T \rightarrow 0$ ), agora com a presença das inúmeras excitações partícula-buraco da banda de condução acopladas à impureza via a hibridização, o gap desaparece e assim o calor específico retrata a linearidade imposta pelo gás de elétrons: temos um comportamento de líquido de Fermi a baixas temperaturas, ou seja, $C_{i m p}=\gamma T$. O mesmo se dá para a suscetibilidade de carga, ou seja, $k_{B} T \chi_{i m p}$ também é linear em $T$, para $T \rightarrow 0$, a exemplo dos casos com impureza magnética, como nos modelos de Kondo ou Anderson. O 


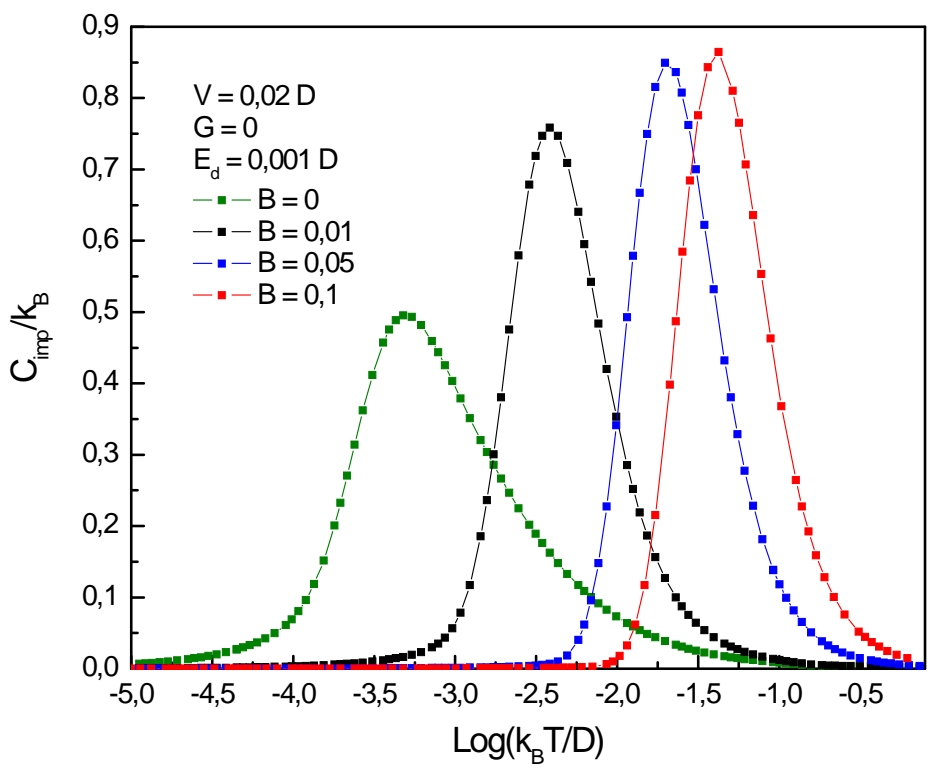

Figura 4.5: Curvas do calor específico do MFK para diversos valores de $B$, sendo $V=0,02 D, G=0$ e $E_{d}=0,001 D$.

comportamento linear a baixas temperaturas está bem evidenciado na Fig. (4.7).

Em decorrência desta linearidade, podemos definir o análogo da razão de Wilson

$$
R=\frac{\pi^{2}}{3} \frac{k_{B} T \chi_{i m p}}{C_{i m p} / k_{B}}
$$

mas que ao invés da suscetibilidade magnética contém a de carga. Para valores de $B$ até $0,1, R$ parece independer de $B$ e alcançar o valor unitário. Para valores de $B>0,1$ as oscilações espúrias devido à discretização da banda não permitem uma conclusão definitiva sobre essa dependência. Esse ponto merece ser mais bem explorado no futuro.

\subsection{4 $G \neq 0$ e $V \neq 0$}

Esta situação mais geral não difere qualitativamente da anterior com $G=0$ e $V \neq 0$. Exemplificamos alguns casos nas Fig. (4.8) e Fig. (4.9) por completeza. Também aqui, em baixas 


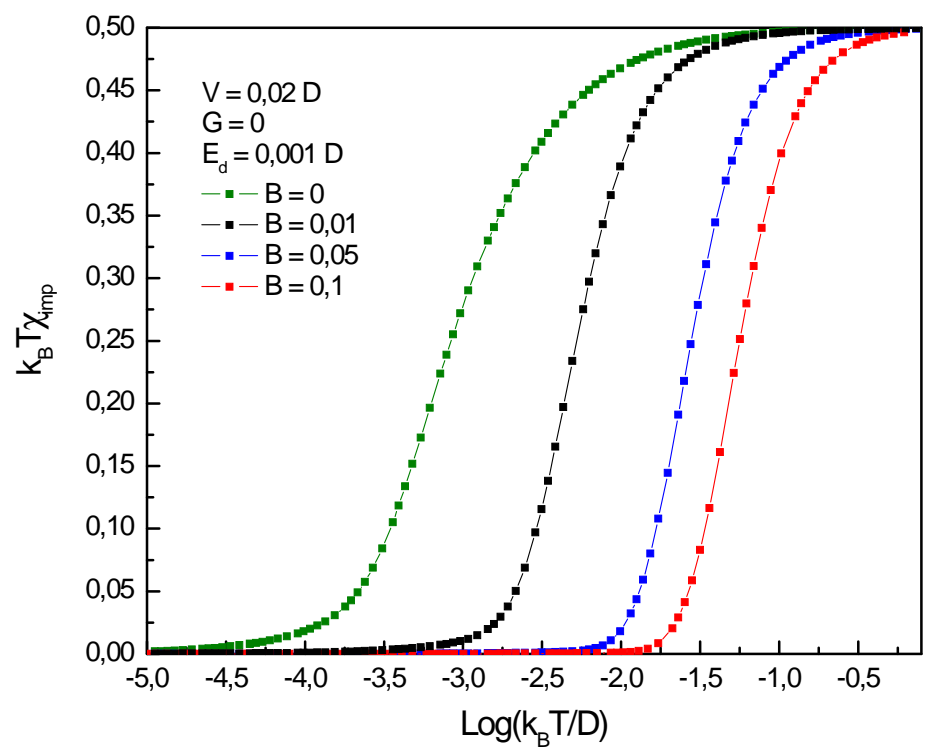

Figura 4.6: Curvas da suscetibilidade de carga do MFK para diversos valores de $B$, sendo $V=0,02 D$, $G=0$ e $E_{d}=0,001 D$.

temperaturas temos um líquido de Fermi com razão de Wilson próxima à unidade.

Tanto neste caso, como no anterior com $G=0$ e $V \neq 0$, a dependência de $R$ com $B$ deve se

dar através do termo $\zeta_{0} f_{0 \pm}^{\dagger} f_{0 \pm}$ presente em $H_{N}$. Isto porque $\zeta_{0} \approx B$ e a combinação $f_{0 \pm}^{\dagger} f_{0 \pm}$ forma um operador marginal no contexto do GRN.[2] Um operador relevante (ou irrelevante) nesse contexto significa que seus elementos de matriz crescem (ou decrescem) à medida que iteramos a transformação do GRN; em outras palavras, aumentamos $N$ na Eq. (4.13). Um operador marginal pode ter seus elementos de matriz fracamente dependentes da iteração $N$; somente um cálculo detalhado pode revelar se no modelo de FK essa marginalidade leva a uma dependência substancial com $B$.

\subsection{Possíveis Generalizações}

A abordagem acima para o MFK pode ser perfeitamente implementada em situações mais realistas, como por exemplo, com a introdução de spin na dinâmica, ou a presença de tunelamento 


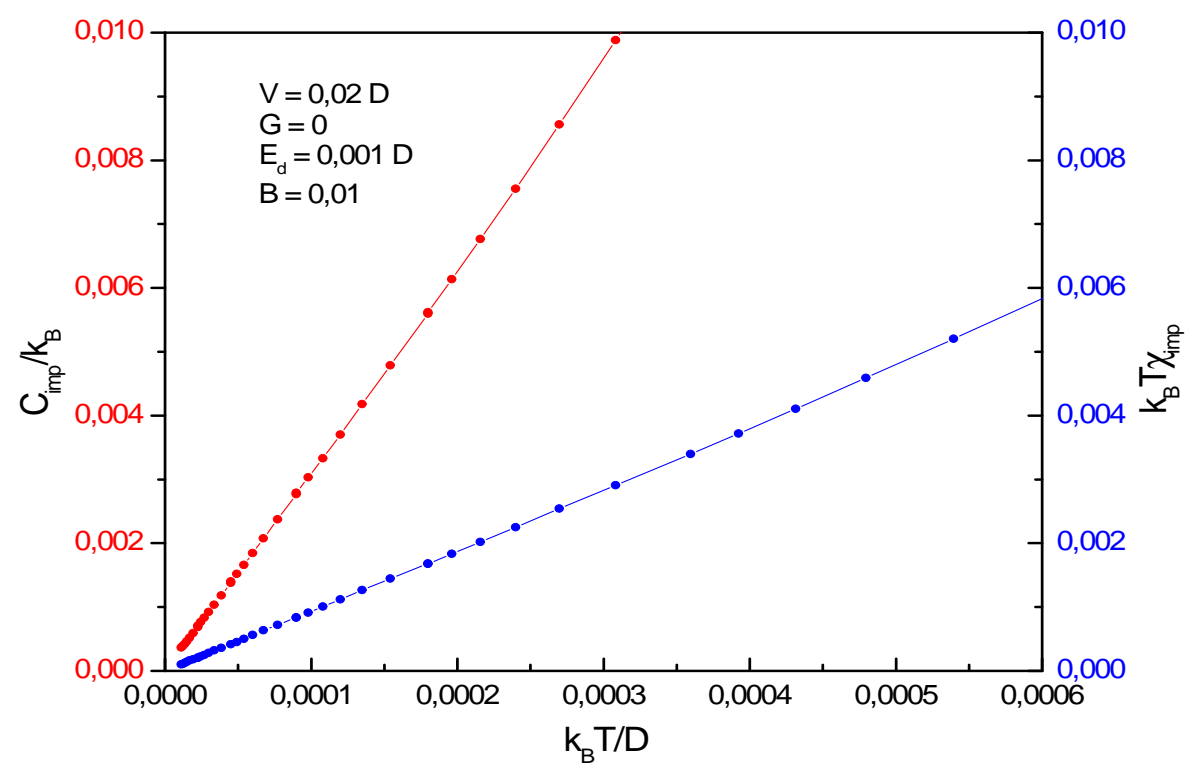

Figura 4.7: Curvas do calor específico (em vermelho) e da susceptibilidade (em azul) num intervalo em baixa temperatura evidenciando o comportamento linear de ambas grandezas para o caso em que $G=0$, sendo $E_{d}=0,001 D$ e $V=0,02 D$.

direto entre os dois orbitais $d_{1}$ e $d_{2}$. Poderíamos ainda não ter projetado a densidade $\rho(\epsilon)$ e o termo $S=\sin (k R) /(k R)$ na esfera de Fermi, como fizemos. Levando em conta essas possibilidades teríamos um modelo mais rico, mas ao mesmo tempo com mais parâmetros livres, o que tornaria a discussão mais elaborada.

Deixamos isso para eventual necessidade em se estudar um sistema físico específico em que esses parâmetros sejam relevantes. 


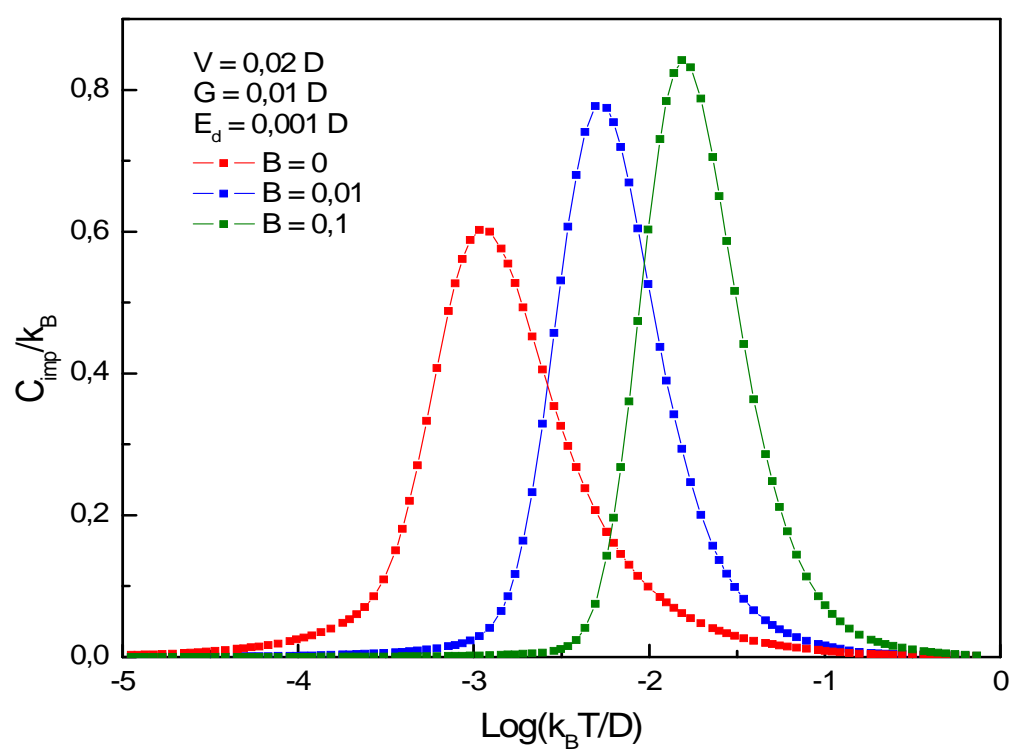

Figura 4.8: Curvas do calor específico para o calor específico do MFK para três valores de campo, sendo $G, V$, e $E_{d}$ simultaneamente diferentes de zero.

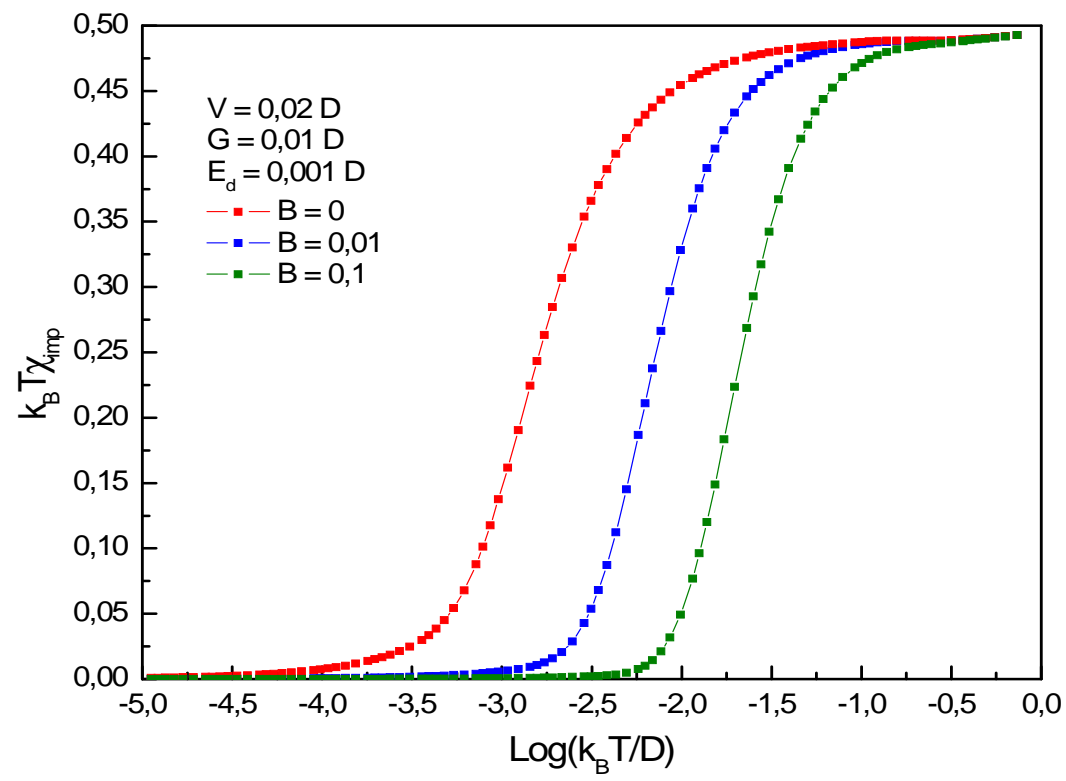

Figura 4.9: Curvas da suscetibilidade de carga para o calor específico do MFK para três intensidades de campo, para valores de $G, V$, e $E_{d}$ simultaneamente diferentes de zero. 


\section{Capítulo 5}

\section{Modelo de Kondo de Dois Canais}

A aplicação de campo magnético a um modelo não trivial e com spin seria natural na sequência deste trabalho. Como nos foi sugerido pelo Dr. Daniel Cox, o estudo da termodinâmica do Modelo de Kondo de Dois Canais (MK2C), na presença de campo magnético, seria de muito valor. Inclusive, trabalho do qual Cox fez parte aplica o GRN a este modelo na presença de campo [29]. Mas como não dispunham do procedimento de discretização a dois parâmetros $(\Lambda$ e $z)$, limitaram-se a estudar os pontos fixos do modelo em questão não tendo determinado nenhuma propriedade termodinâmica.

De fato, mesmo com os dois parâmetros de discretização, veremos adiante que para esse modelo a suavização das curvas termodinâmicas é crítica mesmo sem o campo magnético aplicado. Com campo externo $B$ antecipamos uma grande dificuldade numérica em se tratando deste modelo: devido à quebra da simetria partícula-buraco, o operador isospin não é mais conservado e isso acarreta em custos computacionais mais elevados na diagonalização. Para amenizar este relevante inconveniente, será necessário introduzir cortes mais drásticos nas energias altas, o que certamente acarretará oscilações espúrias mais acentuadas. Sendo assim, optamos por estudar o modelo inicialmente sem o campo para adquirir mais experiência na suavização das curvas termodinâmicas preparando o caminho para um futuro trabalho de aplicação do GRN para campo externo num modelo de canais. 
O modelo Kondo [4] bem como o de Anderson [6] para impureza magnética em meio metálico prevêem comportamento de líquido de Fermi a baixas temperaturas. Isto é, calor específico proporcional a $T$ e suscetibilidade magnética constante, para temperaturas bem baixas. Embora muitos materiais exibam tal comportamento, o que confere grande importância a esses modelos, existem outros que se desviam bastante desses comportamentos assintóticos.

Nozières e Blandin [7], a fim de tornar o modelo de Kondo mais realista, introduziram o conceito de canais na banda de condução, que fazem o papel de números quânticos que conferem mais graus de liberdade ao sistema. Com isso, puderam explicar porque determinados materiais exibiam propriedades não-líquido de Fermi a baixas temperaturas. O modelo de dois canais fornece calor específico e suscetibilidade proporcionais a $\ln T$ e a $(\ln T) / T$, respectivamente, como o exibem certas classes de materiais tais como os férmions pesados.

\subsection{Modelo e Simetrias}

O Hamiltoniano que define o Modelo de Kondo usual é o seguinte :

$$
H=\sum_{\vec{k}, \sigma} \epsilon \vec{k} c_{\vec{k} \sigma}^{\dagger} c_{\vec{k} \sigma}+J \vec{s} \cdot \vec{S}_{I},
$$

onde $\vec{s}$ é o spin do elétron de condução e $\vec{S}_{I}$ é o spin da impureza.

O Modelo de Kondo [4] (ou modelo s-d) considera um spin localizado $\vec{S}_{I}$ (com origem em estados $d$ ou $f$ ) embebido em um mar de elétrons de condução (normalmente, elétrons $s$ ), interagindo via o termo de troca $J \vec{s} \cdot \vec{S}$, com $\vec{s}=\sum c_{\vec{k}, \mu}^{\dagger} \vec{\sigma}_{\mu, \nu} c_{\overrightarrow{k^{\prime}, \nu}}$ sendo o spin de condução no sítio da impureza (a soma sobre os índices é implícita). Nozières and Blandin [7] generalizaram o modelo de Kondo incluindo mais graus de liberdade à banda de condução, que são denominados de canais da banda. Com isso, o termo de troca do modelo fica $\sum_{\alpha} J_{\alpha} \vec{s}_{\alpha} \cdot \vec{S}_{I}$, onde $\vec{S}_{I}$ é o spin localizado da impureza e

$$
\vec{s}_{\alpha}=\sum_{\vec{k}, \overrightarrow{k^{\prime}, \mu, \nu}} c_{\vec{k}, \mu, \alpha}^{\dagger} \vec{\sigma}_{\mu, \nu} c_{\vec{k}^{\prime}, \nu, \alpha},
$$


é o spin do estado de condução no sítio da impureza. Os estados de condução são rotulados por $\alpha=1, \ldots, K$. Neste trabalho iremos considerar o caso de dois canais $(K=2)$, um dos mais importantes na literatura, pois, suas propriedades são de não líquido de Fermi. O Hamiltoniano, portanto, se define por

$$
H=\sum_{\vec{k}, \sigma, \alpha} \epsilon_{k} c_{\vec{k} \sigma \alpha}^{\dagger} c_{\vec{k} \sigma \alpha}+\sum_{\alpha} J_{\alpha} \vec{s}_{\alpha} \cdot \vec{S}_{I}
$$

onde a integral de troca $J_{\alpha}$ pode em geral ser diferente para cada canal.

Este Hamiltoniano será diagonalizado utilizando o GRN. Para o caso de um canal, esse é um procedimento bem conhecido na literatura e segue as linhas originais de Wilson [2]. Optamos aqui por não detalhar os caminhos que levam $H$ ao Hamiltoniano equivalente e reescrito pelo GRN, ou seja, reescrito na base especial dos operadores $f_{n}$ (a base Kondo). Em linhas gerais, segue os desenvolvimentos apresentados nos Cap. 2 e 3 ; detalhes (com $B=0$ ) podem ser encontrados nas teses das Ref. [26, 31, 32]. No entanto, daremos alguns passos relevantes dessa implementação e nos preocuparemos mais com os detalhes pertinentes ao modelo de dois canais, como as simetrias, sua implementação em duas fases, cálculo dos elementos de matriz invariantes e expressões gerais dos elementos de matriz do Hamiltoniano.

No GRN começa-se com a discretização logarítmica da banda de condução, isto é, definindose operadores discretos nos intervalos de energia $\Lambda^{-n-1-z} D \leq|\epsilon| \leq \Lambda^{-n-z} D$, com $\Lambda>1$ e $D$, a semi-largura da banda. Uma base de estados $\left\{f_{n, \sigma, \alpha}\right\}$ é introduzida de tal forma que $f_{n, \sigma, \alpha}$ se acople apenas com $f_{n \pm 1, \sigma, \alpha}$. O ponto de partida é o operador

$$
f_{0, \sigma, \alpha}=\int_{-D}^{D} d \epsilon c_{\epsilon, \sigma, \alpha} / \sqrt{2 D}
$$

o mais localizado ao redor da impureza. Assim, o Hamiltoniano $H$ torna-se

$$
H=D\left(1+\Lambda^{-1}\right) / 2 \lim _{N \rightarrow \infty} \Lambda^{-\frac{N+1}{2}-z+1} H_{N}
$$


com

$$
H_{N}=\Lambda^{\frac{N-1}{2}}\left\{\sum_{\substack{n=0 \\ \sigma, \alpha}}^{N-1} \epsilon_{n, \sigma}\left(f_{n \sigma \alpha}^{\dagger} f_{n+1 \sigma \alpha}+H . c .\right)+\sum_{\mu, \mu^{\prime}, \alpha} \tilde{J}_{\alpha} f_{0, \mu, \alpha}^{\dagger} \vec{\sigma}_{\mu, \mu^{\prime}} \cdot f_{0, \mu^{\prime}, \alpha} \vec{S}_{I}\right\}
$$

segundo a notação $\tilde{J}_{\alpha} \equiv \frac{2}{1+\Lambda^{-1}} \frac{J_{\alpha}}{D}$.

A mais simples simetria desse modelo é a conservação da carga da banda (ou número de elétrons) em cada canal:

$$
\hat{Q}_{N, \alpha}=\sum_{n, \mu}^{N} f_{n, \mu, \alpha}^{\dagger} f_{n, \mu, \alpha} .
$$

É imediato mostrar que $\left[H_{N}, \hat{Q}_{N, \alpha}\right]=0$. A transformação que deixa $H$ e $\hat{Q}$ invariantes é $f_{n, \sigma, \alpha} \rightarrow$ $e^{i \theta} f_{n, \sigma, \alpha}$

O modelo também exibe simetria partícula-buraco, ou seja, é invariante sob a operação

$$
f_{n, \sigma, \alpha} \rightarrow(-1)^{n} \epsilon_{\sigma, \mu} f_{n, \mu, \alpha}^{\dagger}
$$

onde $\epsilon$ é um tensor antissimétrico $\left(\epsilon_{\mu, \nu}=-\epsilon_{\nu, \mu}\right)$. Para spin $1 / 2, \epsilon_{\uparrow, \downarrow}=-\epsilon_{\downarrow, \uparrow}$ e a transformação pode ser escrita como $f_{\mu} \rightarrow(-2 \mu) f_{-\mu}^{\dagger}$. É fácil escrever um operator invariante sob essa transformação:

$$
\begin{aligned}
\hat{J}_{\alpha}^{z} & =\frac{1}{2} \sum_{n, \sigma}\left(f_{n, \uparrow, \alpha}^{\dagger} f_{n, \uparrow, \alpha}-f_{n, \downarrow, \alpha} f_{n, \downarrow, \alpha}^{\dagger}\right) \\
& =\frac{1}{2} \sum_{n, \sigma}\left(f_{n, \sigma, \alpha}^{\dagger} f_{n, \sigma, \alpha}-1 / 2\right)
\end{aligned}
$$

portanto, proporcional ao operator carga $\hat{Q}_{N, \alpha}$; ele também comuta com $H$. O que a conservação de $\hat{J}_{\alpha}^{z}$ nos diz é que os estados com autovalores $j_{\alpha}^{z} \mathrm{e}-j_{\alpha}^{z}$ são degenerados. No entanto, é possível escrever um operador vetorial com álgebra $S U(2)$ (ou álgebra de spin 1/2) com $\hat{J}_{\alpha}^{z}$ sua componente $z[24]$ :

$$
\left[\hat{J}_{\alpha}^{+}, \hat{J}_{\alpha}^{z}\right]=\hat{J}_{\alpha}^{+} \quad \text { e } \quad\left[\hat{J}_{\alpha}^{+}, \hat{J}_{\alpha}^{-}\right]=2 \hat{J}_{\alpha}^{z}
$$


Esse é o operador carga axial ou isospin:

$$
\begin{aligned}
\hat{J}_{\alpha}^{z} & =\frac{1}{2} \sum_{n=0}^{N}\left(f_{n \uparrow \alpha}^{\dagger} f_{n \uparrow \alpha}-f_{n \downarrow \alpha} f_{n \downarrow \alpha}^{\dagger}\right) \\
\hat{J}_{\alpha}^{+} & =\sum_{n=0}^{N}(-1)^{n} f_{n \uparrow \alpha}^{\dagger} f_{n \downarrow \alpha}^{\dagger} \\
\hat{J}_{\alpha}^{-} & =\sum_{n=0}^{N}(-1)^{n} f_{n \downarrow \alpha} f_{n \uparrow \alpha} .
\end{aligned}
$$

É fácil mostrar que

$$
\left[H, \hat{J}_{\alpha}^{z}\right]=0 \quad \text { and } \quad\left[H, \hat{J}_{\alpha}^{2}\right]=0
$$

Note que o isospin é conservado em cada canal. O que o isospin nos diz é que os $2 j_{\alpha}+1$ estados com $j_{\alpha}^{z}=-j,-j+1, \ldots, j-1, j$ são degenerados.

O Hamiltoniano também comuta com os operadores de spin total:

$$
\hat{S}_{T}^{2}=\left(\sum_{\alpha} \vec{s}_{\alpha}+\vec{S}_{I}\right)^{2}
$$

e sua componente $z$ :

$$
\hat{S}^{z}=\frac{1}{2} \sum_{n, \alpha}\left(f_{n, \uparrow, \alpha}^{\dagger} f_{n, \uparrow, \alpha}-f_{n, \downarrow, \alpha}^{\dagger} f_{n, \downarrow, \alpha}\right)+S_{I}^{z} .
$$

Com essas comutações, podemos usar os auto-estados dos operadores $J_{1}^{2}, J_{1 z}, J_{2}^{2}, J_{2 z}, S_{z}^{2}$ e $S_{z}$ para rotular os estados de base, denotados por $\left|j_{1}, j_{1 z}, j_{2}, j_{2 z}, s, s^{z}\right\rangle$, para se contruir a representação matricial de $H$.

\subsection{A transformação do Grupo de Renormalização em duas fases}

Do Hamiltoniano $H_{N}$ nós podemos escrever a seguinte transformação do GRN

$$
H_{N}=\sqrt{\Lambda} H_{N-1}+\sum_{\sigma, \alpha} \xi_{N-1}^{(z)}\left(f_{N-1, \sigma, \alpha}^{\dagger} f_{N, \sigma, \alpha}+H . c .\right)
$$


$\operatorname{com} \xi_{n}^{(z)}=\Lambda^{n / 2} \epsilon_{n}^{(z)}$, sendo $\epsilon_{n}^{(z)}$ os coeficientes $t_{n}(B=0)$ do Cap. 2. Essa relação de recorrência é que permite que se diagonalize $H_{N}$ iterativamente, iniciando-se o processo com

$$
H_{0}=\Lambda^{-1 / 2} \sum_{\alpha} \tilde{J}_{\alpha} f_{0 \mu \alpha}^{\dagger} \vec{\sigma}_{\mu \nu} f_{0 \nu \alpha} \cdot \vec{S}_{I}
$$

A relação acima define a transformação usual do GRN. É possível trabalhar com uma transformação implementada em duas fases, ou etapas, $A$ e $B$ [10]:

$$
\begin{aligned}
H_{N, A} & =H_{N-1, B}+\sum_{\sigma} \frac{\xi_{N-1, A}^{(z)}}{\sqrt{\Lambda}}\left(f_{N-1, \sigma, A}^{\dagger} f_{N, \sigma, A}+H . c .\right) \\
H_{N, B} & =\sqrt{\Lambda} H_{N, A}+\sum_{\sigma} \xi_{N-1, B}^{(z)}\left(f_{N-1, \sigma, B}^{\dagger} f_{N, \sigma, B}+H . c .\right)
\end{aligned}
$$

Em nosso problema nós identificamos a fase $A$ com o canal 1 e a fase $B$ com o canal 2; cada iteração $N$ se inicia na fase $A$ diagonalizando-se $H_{N, A}$, e é completada apenas ao final da fase $B$ diagonalizando-se $H_{N, B}$. As seções seguintes detalham como essa implementação é feita para o modelo MK2C. Para o modelo de Anderson de duas impurezas a Ref. [10] utiliza esquema análogo para o cálculo da densidade espectral.

\subsection{Diagonalização Iterativa}

A base de estados para se construir $H_{N, A}$ é construída do produto direto dos autovetores de $H_{N-1, B}$ com os seguintes estados do canal $A$ : $|\mu\rangle_{N}^{A} \equiv|0\rangle,|\uparrow\rangle,|\downarrow\rangle$, ou $|\uparrow \downarrow\rangle$. O produtos são tais que, após combinados utilizando-se os coeficientes de Clebsh-Gordan [30], resultam nos seguintes autovetores de $J_{A}^{2}, J_{A z}, J_{B}^{2}, J_{B, z}, S^{2}$ e $S_{z}$ (notação: $\left|j_{A}, j_{A z}, j_{B}, j_{B, z}, s, s^{z} ; \mu\right\rangle \equiv\left|j_{A}, j_{A z}, j_{B}, j_{B, z}, s, s^{z}\right\rangle_{N-1}^{B} \otimes$ 
$\left.|\mu\rangle_{N}^{A}\right)$

$$
\begin{aligned}
|1\rangle_{N}^{A} & =\sqrt{\frac{j_{A}-j_{A}^{z}}{2 j_{A}}}\left|j_{A}-\frac{1}{2}, j_{A}^{z}+\frac{1}{2} ; j_{B}, j_{B}^{z} ; s, s^{z} ; 0\right\rangle \\
& +(-1)^{N} \sqrt{\frac{j_{A}+j_{A}^{z}}{2 j_{A}}}\left|j_{A}-\frac{1}{2}, j_{A}^{z}-\frac{1}{2} ; j_{B}, j_{B}^{z} ; s, s^{z} ; \uparrow \downarrow\right\rangle \\
|2\rangle_{N}^{A} & =\sqrt{\frac{s-s^{z}}{2 s}}\left|j_{A}, j_{A}^{z} ; j_{B}, j_{B}^{z} ; s-\frac{1}{2}, s^{z}+\frac{1}{2} ; \downarrow\right\rangle \\
& +\sqrt{\frac{s+s^{z}}{2 s}}\left|j_{A}, j_{A}^{z} ; j_{B}, j_{B}^{z} ; s-\frac{1}{2}, s^{z}-\frac{1}{2} ; \uparrow\right\rangle \\
|3\rangle_{N}^{A} & =\sqrt{\frac{s+s^{z}+1}{2 s+2}}\left|j_{A}, j_{A}^{z} ; j_{B}, j_{B}^{z} ; s+\frac{1}{2}, s^{z}+\frac{1}{2} ; \downarrow\right\rangle \\
& -\sqrt{\frac{s-s^{z}+1}{2 s+2}}\left|j_{A}, j_{A}^{z} ; j_{B}, j_{B}^{z} ; s+\frac{1}{2}, s^{z}-\frac{1}{2} ; \uparrow\right\rangle \\
|4\rangle_{N}^{A} & =\sqrt{\frac{j_{A}+j_{A}^{z}+1}{2 j_{A}+2}}\left|j_{A}+\frac{1}{2}, j_{A}^{z}+\frac{1}{2} ; j_{B}, j_{B}^{z} ; s, s^{z} ; 0\right\rangle \\
& -(-1)^{N} \sqrt{\frac{j_{A}-j_{A}}{2 j_{A}+2}}\left|j_{A}+\frac{1}{2}, j_{A}^{z}-\frac{1}{2} ; j_{B}, j_{B}^{z} ; s, s^{z} ; \uparrow \downarrow\right\rangle .
\end{aligned}
$$

Estes vetores de base $|i\rangle_{N}^{A}$ se diferenciam uns dos outros quanto às diferentes possibilidades de se combinar os autovetores de $H_{N-1, B}$ com os estados $|\mu\rangle_{N}^{A}$ das quais se originaram de modo a compartilharem aqueles mesmos números quânticos, $j_{A}^{2}, j_{A z}, j_{B}^{2}, j_{B, z}, s^{2}, s_{z}$, na iteração $N$, fase $A$. Historicamente, denominamos de tipo a cada uma destas diferentes gêneses, assim entendidas em termos das variações que sofrem os números quânticos dos autovetores ao se combinarem com os estados de uma partícula para gerarem - daí a expressão gênese - os correspondentes estados de base da fase seguinte. Tal uso é recorrente neste trabalho, como verificará o leitor quando formos construir os vetores de base da iteração $N$, fase $B$ mais adiante.

Notemos que $\left[H, \hat{J}_{\alpha}^{2}\right]=0$ e $\left[H, \hat{J}_{\alpha}^{z}\right]=0$, de sorte que os autovalores de $H$ não dependem de $j_{\alpha}^{z}$. Naturalmente, aproveitamo-nos disso arbitrando $j^{z}=j$ naquele conjunto de vetores de base anterior. Da mesma forma, sem campo magnético aplicado, os autovalores não dependem da componente $z$ do spin total, o que nos permite impor semelhantemente que $s_{z}=s$ conferindo uma aparência ligeiramente 
mais amistosa aos vetores-tipos em questão:

$$
\begin{aligned}
& |1\rangle_{N}^{A}=(-1)^{N}\left|j_{A}-\frac{1}{2}, j_{A}-\frac{1}{2} ; j_{B}, j_{B} ; s, s ; \uparrow \downarrow\right\rangle \\
& |2\rangle_{N}^{A}=\left|j_{A}, j_{A} ; j_{B}, j_{B} ; s-\frac{1}{2}, s-\frac{1}{2} ; \uparrow\right\rangle \\
& |3\rangle_{N}^{A}=\sqrt{\frac{2 s+1}{2 s+2}}\left|j_{A}, j_{A} ; j_{B}, j_{B} ; s+\frac{1}{2}, s+\frac{1}{2} ; \downarrow\right\rangle \\
& -\sqrt{\frac{1}{2 s+2}}\left|j_{A}, j_{A} ; j_{B}, j_{B} ; s+\frac{1}{2}, s-\frac{1}{2} ; \uparrow\right\rangle \\
& |4\rangle_{N}^{A}=\sqrt{\frac{2 j_{A}+1}{2 j_{A}+2}}\left|j_{A}+\frac{1}{2}, j_{A}+\frac{1}{2} ; j_{B}, j_{B} ; s, s ; 0\right\rangle \\
& -(-1)^{N} \sqrt{\frac{1}{2 j_{A}+2}}\left|j_{A}+\frac{1}{2}, j_{A}-\frac{1}{2} ; j_{B}, j_{B} ; s, s ; \uparrow \downarrow\right\rangle \text {. }
\end{aligned}
$$

Os elementos diagonais de $H_{N A}$ são calculados via Eq. (5.13) da seguinte maneira (sendo $|t i p o\rangle$, vale a pena insistir, um dos membros de um grupo de vetores de base - há no máximo quatro em cada grupo - que compartilham o mesmo conjunto de números quânticos, diferenciando-se entre si quanto à combinação de vetores da fase anterior que os originou):

$$
\begin{aligned}
{ }_{N}^{A}\left\langle\text { tipo }\left|H_{N, A}\right| \text { tipo }\right\rangle_{N}^{A} & =\left\langle N-1, B, r ; \mu_{\text {tipo }}\left|H_{N-1, B}\right| N-1, B, r ; \mu_{\text {tipo }}\right\rangle \\
& +\sum_{\sigma} \frac{\xi_{N-1, A}^{(z)}}{\sqrt{\Lambda}}\left\langle N-1, B, r ; \mu_{\text {tipo }}\left|\left(f_{N-1, \sigma, A}^{\dagger} f_{N, \sigma, A}+H . c .\right)\right| N-1, B, r ; \mu_{\text {tipo }}\right\rangle \\
& =\left\langle N-1, B, r\left|H_{N-1, B}\right| N-1, B, r\right\rangle
\end{aligned}
$$

onde $|N-1, B, r\rangle$ é o r-ésimo auto-estado de $H_{N-1, B}$, e $\left|\mu_{t i p o}\right\rangle$ representa um dos estados $|\mu\rangle_{N}^{A} \equiv|0\rangle$, $|\uparrow\rangle,|\downarrow\rangle,|\uparrow \downarrow\rangle$, respectivamente para tipo $=1,2,3$ ou 4 . Percebamos que os elementos diagonais de $H_{N, A}$ dependem apenas dos autovalores de $H_{N-1, B}$.

Os elementos não diagonais de $H_{N A}$ são calculados assim:

$$
\begin{aligned}
N_{, A}\left\langle\text { tipo }\left|H_{N, A}\right| \text { tipo }^{\prime}\right\rangle_{N, A} & =\left\langle N-1, B, r ; \mu_{\text {tipo }}\left|H_{N-1, B}\right| N-1, B, r^{\prime} ; \mu_{\text {tipo' }}\right\rangle \\
& +\sum_{\sigma} \frac{\xi_{N-1, A}^{(z)}}{\sqrt{\Lambda}}\left\langle N-1, B, r ; \mu_{\text {tipo }}\left|\left(f_{N-1, \sigma, A}^{\dagger} f_{N, \sigma, A}+H . c .\right)\right| N-1, B, r^{\prime} ; \mu_{\text {tipo }}\right\rangle \\
& =\sum_{\sigma} \frac{\xi_{N-1, A}^{(z)}}{\sqrt{\Lambda}}\left\langle\mu_{t i p o}\left|f_{N, \sigma, A}^{\dagger}\right| \mu_{t i p o^{\prime}}\right\rangle\left\langle N-1, B, r\left|f_{N-1, \sigma, A}\right| N-1, B, r^{\prime}\right\rangle,(5.17)
\end{aligned}
$$


sendo os elementos $\left\langle N-1, B, r\left|f_{N-1, \sigma, A}\right| N-1, B, r^{\prime}\right\rangle$ determinados na iteração $N-1$, fase $B$. Os demais, $\left\langle\mu_{t i p o}\left|f_{N, \sigma, A}^{\dagger}\right| \mu_{t i p o^{\prime}}\right\rangle$, são iguais à unidade se $($ tipo, tipo' $)=(2,1),(3,1)$ or $(4,2)$; serão iguais a -1 se $(4,3)$, e zero se ocorre qualquer outro par (tipo, tipo'). Então nós temos apenas quatro tipos de elementos de matriz de $H_{N A}$. Usando a Eq. (5.15) temos que

$$
\begin{aligned}
\left\langle 2\left|H_{N}^{A}\right| 1\right\rangle_{N}^{A} & =\frac{\xi_{N-1, A}^{(z)}}{\sqrt{\Lambda}}(-1)^{N} \sqrt{\frac{2 s}{2 s+1}}\left\langle j_{A}, j_{B}, s-1 / 2\left\|f_{N-1}^{A}\right\| j_{A}-1 / 2, j_{B}, s\right\rangle_{N-1}^{B} \\
\left\langle 3\left|H_{N}^{A}\right| 1\right\rangle_{N}^{A} & =\frac{-\xi_{N-1, A}^{(z)}}{\sqrt{\Lambda}}(-1)^{N} \sqrt{\frac{2 s+2}{2 s+1}}\left\langle j_{A}, j_{B}, s+1 / 2\left\|f_{N-1}^{A}\right\| j_{A}-1 / 2, j_{B}, s\right\rangle_{N-1}^{B} \\
\left\langle 4\left|H_{N}^{A}\right| 2\right\rangle_{N}^{A} & =\frac{\xi_{N-1, A}^{(z)}}{\sqrt{\Lambda}} \sqrt{\frac{2 j_{A}+2}{2 j_{A}+1}}\left\langle j_{A}+1 / 2, j_{B}, s\left\|f_{N-1}^{A}\right\| j_{A}, j_{B}, s-1 / 2\right\rangle_{N-1}^{B} \\
\left\langle 4\left|H_{N}^{A}\right| 3\right\rangle_{N}^{A} & =\frac{\xi_{N-1, A}^{(z)}}{\sqrt{\Lambda}} \sqrt{\frac{2 j_{A}+2}{2 j_{A}+1}}\left\langle j_{A}+1 / 2, j_{B}, s\left\|f_{N-1}^{A}\right\| j_{A}, j_{B}, s+1 / 2\right\rangle_{N-1}^{B},
\end{aligned}
$$

onde as barras duplas nos sanduiches dos elementos de matriz serão justificadas logo a seguir.

Vamos calcular um desses elementos como exemplo (notação: $|j, s ; \uparrow\rangle$ é um auto-estado da iteração $N-1$, fase $B$, com números quânticos $j, s$, mais um spin $\uparrow$ do canal $A$, iteração $N$ ). Nós aqui também escolhemos $j_{\alpha}^{z}=j_{\alpha}$ e $s_{z}=s$ :

$$
\begin{aligned}
\left\langle 2\left|H_{N}^{A}\right| 1\right\rangle_{N}^{A} & =(-1)^{N}\left\langle j_{A}, j_{A}, j_{B}, j_{B}, s-\frac{1}{2}, s-\frac{1}{2} ; \uparrow\left|f_{N-1, \downarrow}^{\dagger} f_{N, \downarrow} A\right| j_{A}-\frac{1}{2}, j_{A}-\frac{1}{2}, j_{B}, j_{B}, s, s ; \uparrow \downarrow\right\rangle_{N}^{A} \\
& =-(-1)^{N}\left\langle j_{A}, j_{A}, j_{B}, j_{B}, s-\frac{1}{2}, s-\frac{1}{2} ; \uparrow\left|f_{N-1, \downarrow}^{\dagger}\right| j_{A}-\frac{1}{2}, j_{A}-\frac{1}{2}, j_{B}, j_{B}, s, s ; \uparrow\right\rangle_{N}^{A} \\
& =(-1)^{N}\left\langle j_{A}, j_{A}, j_{B}, j_{B}, s-\frac{1}{2}, s-\frac{1}{2}\left|f_{N-1, \downarrow}^{\dagger}\right| j_{A}-\frac{1}{2}, j_{A}-\frac{1}{2}, j_{B}, j_{B}, s, s ;\right\rangle_{N-1}^{B} \\
& =(-1)^{N} \sqrt{\frac{2 s}{2 s+1}}\left\langle j_{A}, j_{B}, s-1 / 2|| f_{N-1}^{A}|| j_{A}-1 / 2, j_{B}, s\right\rangle_{N-1}^{B} .
\end{aligned}
$$

A invariância que tornou lícito arbitrarmos valores quaisquer para $j_{\alpha}^{z}$ e $s_{z}$, aliada ao fato de que os operadores como $f_{N-1, \mu, A}^{\dagger}$ possam ser considerados componentes de um tensor, torna conveniente a introdução do conceito de invariante. Este decorre do Teorema de Wigner-Eckart que nos indica o procedimento de isolarmos a parte do elemento de matriz que depende da particular escolha de $j_{\alpha}^{z}$ e $s_{z}$ - o que depende é o elemento de matriz, não os autovalores! - da outra que não depende, daí o sua denominação de invariante. No elemento de matriz que acabamos de calcular, 
fatoramos

$$
\left\langle j_{A}, j_{A}, j_{B}, j_{B}, s-\frac{1}{2}, s-\frac{1}{2}\left|f_{N-1, \downarrow}^{\dagger}\right| j_{A}-\frac{1}{2}, j_{A}-\frac{1}{2}, j_{B}, j_{B}, s, s ;\right\rangle_{N-1}^{B}
$$

numa parte passível de variação conforme a escolha, $\sqrt{\frac{2 s}{2 s+1}}$, e outra a qual chamamos de invariante, $\left\langle j_{A}, j_{B}, s-1 / 2|| f_{N-1}^{A} \| j_{A}-1 / 2, j_{B}, s\right\rangle_{N-1}^{B}$. As barras duplas internas sinalizam este atributo. No Apêndice C, há detalhes precisos desta fatoração.

Note que os invariantes do operador $f^{A}$ são calculados entre auto-estados da fase $B$. Para calcular esses elementos nós precisamos expressar os auto-estados da fase $B$ em termos dos auto-estados da fase $A$.

A próxima seção mostra como os vetores de base da fase $B$ são construídos a partir dos auto-estados da fase $A$.

\subsection{Os vetores de base da fase $\mathrm{B}$}

Pare se construir os vetores de base da iteração $N$, fase $B$, nós temos que combinar os auto-estados da iteração $N$ fase $A$ - chamemos tal iteração $(N, A)$ - com os estados de canal da fase $B,|\mu\rangle_{N}^{B} \equiv|0\rangle,|\uparrow\rangle,|\downarrow\rangle$ ou $|\uparrow \downarrow\rangle$, de maneira que todos sejam auto-estados dos operadores $J_{A}^{2}, J_{A z}, J_{B}^{2}, J_{B z}, S^{2}$ e $S_{z}$. A seguinte lista dá as regras para se obterem todos os estados da fase $B$ a partir daqueles da fase $A$ e identifica cada combinação atribuindo um tipo $\left(j_{A}, j_{B}\right.$ e $s$ são números quânticos da iteração $N$, fase $A$ - aqui será usado o conceito de tipo tal como explicado na seção anterior. Seguindo a nomenclatura tipo : combinação, temos

$$
\begin{aligned}
& 1: j_{A}, j_{B}+\frac{1}{2}, s+0 \\
& 2: j_{A}+0, j_{B}+0, s+\frac{1}{2} \\
& 3: j_{A}+0, j_{B}+0, s-\frac{1}{2} \\
& 4 \quad: \quad j_{A}, j_{B}-\frac{1}{2}, s+0 .
\end{aligned}
$$


Os vetores de base são (notação: $\mid$ tipo $\left.\rangle_{N}^{B}\right)$ :

$$
\begin{aligned}
|1\rangle_{N}^{B} & =\sqrt{\frac{j_{B}-j_{B z}}{2 j_{B}}}\left|j_{A}, j_{A z}, j_{B}-1 / 2, j_{B z}+1 / 2, s, s_{z} ; 0\right\rangle \\
& +(-1)^{N} \sqrt{\frac{j_{B}+j_{B z}}{2 j_{B}}}\left|j_{A}, j_{A z}, j_{B}-1 / 2, j_{B z}-1 / 2, s, s_{z} ; \uparrow \downarrow\right\rangle \\
|2\rangle_{N}^{B} & =\sqrt{\frac{s-s_{z}}{2 s}}\left|j_{A}, j_{A z}, j_{B}, j_{B z}, s-1 / 2, s_{z}+1 / 2 ; \downarrow\right\rangle \\
& +\sqrt{\frac{s+s_{z}}{2 s}}\left|j_{A}, j_{A z}, j_{B}, j_{B z}, s-1 / 2, s_{z}-1 / 2 ; \uparrow\right\rangle \\
|3\rangle_{N}^{B} & =\sqrt{\frac{s+s_{z}+1}{2 s+2}}\left|j_{A}, j_{A z}, j_{B}, j_{B z}, s+1 / 2, s_{z}+1 / 2 ; \downarrow\right\rangle \\
& -\sqrt{\frac{s-s_{z}+1}{2 s+2}}\left|j_{A}, j_{A z}, j_{B}, j_{B z}, s+1 / 2, s_{z}-1 / 2 ; \uparrow\right\rangle \\
|4\rangle_{N}^{B} & =\sqrt{\frac{j_{B}+j_{B z}+1}{2 j_{B}+2}}\left|j_{A}, j_{A z}, j_{B}+1 / 2, j_{B z}+1 / 2, s, s_{z} ; 0\right\rangle \\
& -(-1)^{N} \sqrt{\frac{j_{B}-j_{B z}+1}{2 j_{B}+2}}\left|j_{A}, j_{A z}, j_{B}+1 / 2, j_{B z}-1 / 2, s, s_{z} ; \uparrow \downarrow\right\rangle .
\end{aligned}
$$

Novamente, nós escolhemos $j_{z}=j$ e $s_{z}=s$, e usamos coeficientes de Clebsh-Gordan para soma de $\operatorname{spin} 1 / 2 \operatorname{com} j[30]$.

\subsubsection{Os elementos de matriz de $H_{N}^{B}$}

Usando a segunda linha da Eq. (5.13), e os vetores de base acima, nós calculamos os elementos não diagonais de $H_{N}^{B}$. A álgebra é similar àquela usada em $H_{N, A}$ (simplesmente trocamos $j_{A}$ por $\left.j_{B}\right)$, resultando em

$$
\begin{aligned}
1-\left\langle 2\left|H_{N}^{B}\right| 1\right\rangle_{N}^{B} & =\xi_{N-1, B}^{(z)}(-1)^{N} \sqrt{\frac{2 s}{2 s+1}}\left\langle j_{A}, j_{B}, s-1 / 2\left\|f_{N-1}^{B}\right\| j_{A}, j_{B}-1 / 2, s\right\rangle_{N}^{A} \\
2-\left\langle 3\left|H_{N}^{B}\right| 1\right\rangle_{N}^{B} & =-\xi_{N-1, B}^{(z)}(-1)^{N} \sqrt{\frac{2 s+2}{2 s+1}}\left\langle j_{A}, j_{B}, s+1 / 2\left\|f_{N-1}^{B}\right\| j_{A}, j_{B}-1 / 2, s\right\rangle_{N}^{A} \\
3-\left\langle 4\left|H_{N}^{B}\right| 2\right\rangle_{N}^{B} & =\xi_{N-1, B}^{(z)} \sqrt{\frac{2 j_{B}+2}{2 j_{B}+1}}\left\langle j_{A}, j_{B}+1 / 2, s\left\|f_{N-1}^{B}\right\| j_{A}, j_{B}, s-1 / 2\right\rangle_{N}^{A} \\
4-\left\langle 4\left|H_{N}^{B}\right| 3\right\rangle_{N}^{B} & =\xi_{N-1, B}^{(z)} \sqrt{\frac{2 j_{B}+2}{2 j_{B}+1}}\left\langle j_{A}, j_{B}+1 / 2, s\left\|f_{N-1}^{B}\right\| j_{A}, j_{B}, s+1 / 2\right\rangle_{N}^{A} .
\end{aligned}
$$

Em resumo, para montar $H_{N}^{B}$ nós precisamos dos elementos invariantes $\left\langle\left\|f_{N-1}^{A}\right\|\right\rangle_{N-1}^{B}$ (os 
quais necessitam dos invariantes $\left\langle\| f_{N-1}^{B}||\right\rangle_{N-1}^{B}$ ). Lembremos que, para montar $H_{N}^{B}$ nós precisamos dos invariantes $\left\langle\left\|f_{N-1}^{A}\right\|\right\rangle_{N}^{B}$ (que usam os invariantes $\left\langle\| f_{N-1}^{A}||\right\rangle_{N-1}^{A}$ ).

\subsection{Os elementos invariantes $e m i_{A B}$}

Pelas Eqs. (5.18), para calcular os elementos de matriz $\left\langle\text { tipo }\left|H_{N+1}^{A}\right| t i p o^{\prime}\right\rangle_{N+1}^{A}$ nós precisamos de dois tipos de invariantes, denominados $e m i_{B A} \equiv\left\langle\| f_{N}^{A}||\right\rangle_{N}^{B}$, determinados na iteração $N$ para serem usados na próxma iteração $N+1$ :

$$
\begin{aligned}
& 1-\left\langle j_{A}, j_{B}, s\left\|f_{N}^{A}\right\| j_{A}-1 / 2, j_{B}, s+1 / 2\right\rangle_{N}^{B} \\
& 2-\left\langle j_{A}, j_{B}, s\left\|f_{N}^{A}\right\| j_{A}-1 / 2, j_{B}, s-1 / 2\right\rangle_{N}^{B} .
\end{aligned}
$$

Primeiro de tudo nós escrevemos o $r$-ésimo auto-estado de $H_{N B},|r\rangle_{N}^{B} \equiv\left|j_{A}, j_{B}, s, r\right\rangle_{N}^{B}$, como combinação linear dos k-ésimos estados de base $\mid k$, tipo $\rangle_{N}^{B} \equiv \mid j_{A}, j_{B}, s, k$, tipo $\rangle_{N}^{B} \operatorname{listados}$ em (5.21):

$$
\left.|r\rangle_{N}^{B}=\sum_{k, t i p o} \operatorname{vet}_{t i p o}(k, r) \mid k, \text { tipo }\right\rangle_{N}^{B}
$$

onde $v_{\text {etipo }}(k, r)$ é a k-ésima linha do r-ésimo autovetor de $H_{N}^{B}$; tipo refere-se a um dos estado de base (5.21).

Disso resulta $e m i_{A B}$ como combinação linear dos invariantes da forma $\left\langle j_{A}, j_{B}, s\left\|f_{N}^{A}\right\| j_{A}-\right.$ $\left.1 / 2, j_{B}, s \pm 1 / 2\right\rangle_{N}^{A}$, denominados $e m i_{A A}$. Então, os dois tipos de invariantes que aparecem na Eq. 
(5.23) são:

$$
\begin{aligned}
1 & -\left\langle j_{A}, j_{B}, s, r\left\|f_{N}^{A}\right\| j_{A}-\frac{1}{2}, j_{B}, s+\frac{1}{2}, r^{\prime}\right\rangle_{N}^{B}= \\
& =\frac{\left\langle j_{A}, j_{A}, j_{B}, j_{B}, s, s\left|f_{N \downarrow}^{\dagger A}\right| j_{A}-\frac{1}{2}, j_{A}-\frac{1}{2}, j_{B}, j_{B}, s+\frac{1}{2}, s+\frac{1}{2}\right\rangle_{N}^{B}}{\left\langle j_{A}-\frac{1}{2}, j_{A}-\frac{1}{2} ; \frac{1}{2}, \frac{1}{2} \mid j_{A}, j_{A}\right\rangle\left\langle s+\frac{1}{2}, s+\frac{1}{2} ; \frac{1}{2},-\frac{1}{2} \mid s, s\right\rangle} \\
& =\sum_{k, k^{\prime}}\left\{\operatorname{vet}_{1} \operatorname{vet}_{1}\left\langle j_{A}, j_{B}-\frac{1}{2}, s \| f_{N}^{A}|| j_{A}-\frac{1}{2}, j_{B}-\frac{1}{2}, s+\frac{1}{2}\right\rangle_{N}^{A}\right. \\
& -\operatorname{vet}_{2} \operatorname{vet}_{2} \frac{\sqrt{2 s(2 s+2)}}{2 s+1}\left\langle j_{A}, j_{B}, s-\frac{1}{2}\left\|f_{N}^{A}\right\| j_{A}-\frac{1}{2}, j_{B}, s\right\rangle_{N}^{A} \\
& -\operatorname{vet}_{3} \operatorname{vet}_{3}\left\langle j_{A}, j_{B}, s+\frac{1}{2}\left\|f_{N}^{A}\right\| j_{A}-\frac{1}{2}, j_{B}, s+1\right\rangle_{N}^{A} \\
& +\operatorname{vet}_{4} \operatorname{vet}_{4}\left\langle j_{A}, j_{B}+\frac{1}{2}, s\left\|f_{N}^{A}\right\| j_{A}-\frac{1}{2}, j_{B}+\frac{1}{2}, s+\frac{1}{2}\right\rangle_{N}^{A} \\
& \left.+\operatorname{vet}_{3} \text { vet }_{2} \frac{1}{2 s+1}\left\langle j_{A}, j_{B}, s+\frac{1}{2}\left\|f_{N}^{A}\right\| j_{A}-\frac{1}{2}, j_{B}, s\right\rangle_{N}^{A}\right\}
\end{aligned}
$$

$$
\begin{aligned}
2- & \left\langle j_{A}, j_{B}, s|| f_{N}^{A} \| j_{A}-\frac{1}{2}, j_{B}, s-\frac{1}{2}\right\rangle_{N}^{B}= \\
& \quad \frac{\left\langle j_{A}, j_{A}, j_{B}, j_{B}, s, s\left|f_{N \uparrow}^{A}\right| j_{A}-\frac{1}{2}, j_{A}-\frac{1}{2}, j_{B}, j_{B}, s-\frac{1}{2}, s-\frac{1}{2}\right\rangle_{N}^{B}}{\left\langle j_{A}-\frac{1}{2}, j_{A}-\frac{1}{2} ; \frac{1}{2}, \frac{1}{2} \mid j_{A}, j_{A}\right\rangle\left\langle s-\frac{1}{2}, s-\frac{1}{2} ; \frac{1}{2}, \frac{1}{2} \mid s, s\right\rangle} \\
= & \left\langle j_{A}, j_{A}, j_{B}, j_{B}, s, s\left|f_{N \uparrow}^{A}\right| j_{A}-\frac{1}{2}, j_{A}-\frac{1}{2}, j_{B}, j_{B}, s-\frac{1}{2}, s-\frac{1}{2}\right\rangle_{N}^{B} \\
= & \sum_{k, k^{\prime}}\left\{\operatorname{vet}_{1} \operatorname{vet}_{1}\left\langle j_{A}, j_{B}-\frac{1}{2}, s\left\|f_{N}^{A}\right\| j_{A}-\frac{1}{2}, j_{B}-\frac{1}{2}, s-\frac{1}{2}\right\rangle_{N}^{A}\right. \\
- & \operatorname{vet}_{2} \operatorname{vet}_{2}\left\langle j_{A}, j_{B}, s-\frac{1}{2}\left\|f_{N}^{A}\right\| j_{A}-\frac{1}{2}, j_{B}, s-1\right\rangle_{N}^{A} \\
- & \operatorname{vet}_{3} \operatorname{vet}_{3} \frac{\sqrt{2 s(2 s+2)}}{2 s+1}\left\langle j_{A}, j_{B}, s+\frac{1}{2}\left\|f_{N}^{A}\right\| j_{A}-\frac{1}{2}, j_{B}, s\right\rangle_{N}^{A} \\
+ & \operatorname{vet}_{4} \operatorname{vet}_{4}\left\langle j_{A}, j_{B}+\frac{1}{2}, s\left\|f_{N}^{A}\right\| j_{A}-\frac{1}{2}, j_{B}+\frac{1}{2}, s-\frac{1}{2}\right\rangle_{N}^{A} \\
- & \left.\operatorname{vet}_{2} \operatorname{vet}_{3} \frac{1}{2 s+1}\left\langle j_{A}, j_{B}, s-\frac{1}{2}\left\|f_{N}^{A}\right\| j_{A}-\frac{1}{2}, j_{B}, s\right\rangle_{N}^{A}\right\},
\end{aligned}
$$

onde vet $_{i} \equiv \operatorname{vet}_{i}(k, r)$, com tipo $i$ e vet $_{i}^{\prime} \equiv \operatorname{vet}_{i}\left(k^{\prime}, r^{\prime}\right)$ com tipo $i$.

Para calcular os invariantes $<j_{A}, j_{B}, s, k\left\|f_{N}^{A}\right\| j_{A}^{\prime}, j_{B}^{\prime}, s^{\prime}, l>_{N}^{A}$, que aparecem nessas equações, na iteração $N$ fase $A$ nós escrevemos o $k$-ésimo e o $l$-ésimo auto-estados em termos dos vetores de base. Então, o cálculo involve os invariantes entre vetores de base. Há quatro tipos desses invariantes 
(notação: $<j_{A}, j_{B}, s$, tipo $\left.\left\|f_{N}^{A}\right\| j_{A}-1 / 2, j_{B}, s \pm 1 / 2, \mathbf{t i p o} \mathbf{p}^{\prime}\right\rangle$ ) e são eles

$$
\begin{aligned}
\left\langle j_{A}, j_{B}, s, \mathbf{1}\left\|f_{N}^{A}\right\| j_{A}-\frac{1}{2}, j_{B}, s-\frac{1}{2}, \mathbf{3}\right\rangle_{N}^{A} & =(-1)^{N} \sqrt{\frac{2 s}{2 s+1}} \\
\left\langle j_{A}, j_{B}, s, \mathbf{2}\left\|f_{N}^{A}\right\| j_{A}-\frac{1}{2}, j_{B}, s-\frac{1}{2}, \mathbf{4}\right\rangle_{N}^{A} & =\sqrt{\frac{2 j_{A}}{2 j_{A}+1}} \\
\left\langle j_{A}, j_{B}, s, \mathbf{1}\left\|f_{N}^{A}\right\| j_{A}-\frac{1}{2}, j_{B}, s+\frac{1}{2}, \mathbf{2}\right\rangle_{N}^{A} & =-(-1)^{N} \sqrt{\frac{2 s+2}{2 s+1}} \\
\left\langle j_{A}, j_{B}, s, \mathbf{3}\left\|f_{N}^{A}\right\| j_{A}-\frac{1}{2}, j_{B}, s+\frac{1}{2}, \mathbf{4}\right\rangle_{N}^{A} & =\sqrt{\frac{2 j_{A}}{2 j_{A}+1}} .
\end{aligned}
$$

Como um exemplo, vamos calcular o primeiro:

$$
\begin{aligned}
& \left\langle j_{A}, j_{B}, s, \mathbf{1}\left\|f_{N}^{A}\right\| j_{A}-\frac{1}{2}, j_{B}, s-\frac{1}{2}, \mathbf{3}\right\rangle_{N}^{A}= \\
= & \frac{\left\langle j_{A}, j_{A}, j_{B}, j_{B}, s, s, 1\left|f_{N, \uparrow}^{\dagger A}\right| j_{A}-\frac{1}{2}, j_{A}^{\prime}, j_{B}, j_{B}, s-\frac{1}{2}, s_{z}^{\prime}, 3\right\rangle_{N}^{A}}{\left\langle s-\frac{1}{2}, s_{z}^{\prime} ; \frac{1}{2}, \uparrow \mid s, s\right\rangle\left\langle j_{A}-\frac{1}{2}, j_{A}^{\prime} ; \frac{1}{2}, \frac{1}{2} \mid j_{A}, j_{A}\right\rangle} \\
= & \frac{(-1)^{N} \sqrt{\frac{j_{A}+j_{A}}{2 j_{A}}} \sqrt{\frac{s-\frac{1}{2}+s_{z}^{\prime}+1}{2 s+1}}\left\langle j_{A}-\frac{1}{2}, j_{A}-\frac{1}{2}, j_{B}, j_{B}, s, s ; \uparrow \downarrow\left|f_{N, \uparrow}^{\dagger A}\right| j_{A}-\frac{1}{2}, j_{A}^{\prime}, j_{B}, j_{B}, s, s_{z}^{\prime}+\frac{1}{2} ; \downarrow\right\rangle_{N}^{A}}{\sqrt{\frac{s-\frac{1}{2}+s+\frac{1}{2}}{2 s}} \sqrt{\frac{j_{A}-\frac{1}{2}+j_{A}+\frac{1}{2}}{2 j_{A}}}} \\
= & (-1)^{N} \sqrt{\frac{s-\frac{1}{2}+s_{z}^{\prime}+1}{2 s+1}}\left\langle j_{A}-\frac{1}{2}, j_{A}-\frac{1}{2}, j_{B}, j_{B}, s, s \mid j_{A}-\frac{1}{2}, j_{A}^{\prime}, j_{B}, s, s_{z}^{\prime}+\frac{1}{2}\right\rangle_{N}^{A} \\
= & (-1)^{N} \sqrt{\frac{2 s}{2 s+1}},
\end{aligned}
$$

onde usamos que $s_{z}^{\prime}=s-1 / 2$ e $j_{z}^{\prime}=j-1 / 2$.

\subsection{Os elementos invariantes $e m i_{B A}$}

Inspecionando as Eqs. (5.22), notamos que, para calcular os elementos $\left\langle\text { tipo }\left|H_{N}^{B}\right| t i p o^{\prime}\right\rangle_{N}^{B}$, precisamos de uma espécie de invariante denominada $e m i_{B A} \equiv\left\langle\left\|f_{N}^{B}\right\|\right\rangle_{N}^{A}$ que, por sua vez, subdividese em duas sub-espécies, quais sejam

$$
\begin{aligned}
& 1-\left\langle j_{A}, j_{B}, s\left\|f_{N-1}^{B}\right\| j_{A}, j_{B}-1 / 2, s+1 / 2\right\rangle_{N}^{A} \\
& 2-\left\langle j_{A}, j_{B}, s\left\|f_{N-1}^{B}\right\| j_{A}, j_{B}-1 / 2, s-1 / 2\right\rangle_{N}^{A} .
\end{aligned}
$$


Primeiro nós escrevemos os auto-estados de $H_{N}^{A}$ como combinação linear dos auto-estados de $H_{N-1}^{B}$, usando a Eq. (5.21). Com issso, $e m i_{A B}$ pode ser expresso como combinação dos invariantes da forma $\left\langle j_{A}, j_{B}, s\left\|f_{N-1}^{B}\right\| j_{A}, j_{B}-1 / 2, s \pm 1 / 2\right\rangle_{N-1}^{B}$, denominados $e m i_{B B}$. Uma vez que esse cálculo segue de perto aquele da seção 5.5, nós apenas listamos os resultados:

$$
\begin{aligned}
& 1-\left\langle j_{A}, j_{B}, s, w i\left\|f_{N-1}^{B}\right\| j_{A}, j_{B}-\frac{1}{2}, s+\frac{1}{2}, w j\right\rangle_{N}^{A}=\sum_{i, j}\{ \\
& \text { vet }_{1} \text { vet }_{1}\left\langle j_{A}-\frac{1}{2}, j_{B}, s, i a\left\|f_{N-1}^{B}\right\| j_{A}-\frac{1}{2}, j_{B}-\frac{1}{2}, s+\frac{1}{2}, j a\right\rangle_{N-1}^{B} \\
& - \text { vet }_{2} \text { vet }_{2} \frac{\sqrt{2 s(2 s+2)}}{2 s+1}\left\langle j_{A}, j_{B}, s-\frac{1}{2}\left\|f_{N-1}^{B}\right\| j_{A}, j_{B}-\frac{1}{2}, s\right\rangle_{N-1}^{B} \\
& - \text { vet }_{3} \text { vet }_{3}\left\langle j_{A}, j_{B}, s+\frac{1}{2}\left\|f_{N-1}^{B}\right\| j_{A}, j_{B}-\frac{1}{2}, s+1\right\rangle_{N-1}^{B} \\
& +\operatorname{vet}_{4} \operatorname{vet}_{4}\left\langle j_{A}+\frac{1}{2}, j_{B}, s\left\|f_{N-1}^{B}\right\| j_{A}+\frac{1}{2}, j_{B}-\frac{1}{2}, s+\frac{1}{2}\right\rangle_{N-1}^{B} \\
& \left.+ \text { vet }_{3} \text { vet }_{2} \frac{1}{2 s+1}\left\langle j_{A}, j_{B}, s+\frac{1}{2}\left\|f_{N-1}^{B}\right\| j_{A}, j_{B}-\frac{1}{2}, s\right\rangle_{N-1}^{B}\right\} ; \\
& 2-\left\langle j_{A}, j_{B}, s\left\|f_{N-1}^{B}\right\| j_{A}, j_{B}-\frac{1}{2}, s-\frac{1}{2}\right\rangle_{N}^{A}=\sum_{k, k^{\prime}}\{ \\
& \text { vet }_{1} \operatorname{vet}_{1}\left\langle j_{A}-\frac{1}{2}, j_{B}, s\left\|f_{N-1}^{B}\right\| j_{A}-\frac{1}{2}, j_{B}-\frac{1}{2}, s-\frac{1}{2}\right\rangle_{N-1}^{B} \\
& \text { - } \text { vet }_{2} \text { vet }_{2}\left\langle j_{A}, j_{B}, s-\frac{1}{2}\left\|f_{N-1}^{B}\right\| j_{A}, j_{B}-\frac{1}{2}, s-1\right\rangle_{N-1}^{B} \\
& - \text { vet }_{3} \text { vet }_{3} \frac{\sqrt{2 s(2 s+2)}}{2 s+1}\left\langle j_{A}, j_{B}, s+\frac{1}{2}\left\|f_{N-1}^{B}\right\| j_{A}, j_{B}-\frac{1}{2}, s\right\rangle_{N-1}^{B} \\
& + \text { vet }_{4} \text { vet }_{4}\left\langle j_{A}+\frac{1}{2}, j_{B}, s\left\|f_{N-1}^{B}\right\| j_{A}+\frac{1}{2}, j_{B}-\frac{1}{2}, s-\frac{1}{2}\right\rangle_{N-1}^{B} \\
& \text { - } \left.\text { vet }_{2} \text { vet }_{3} \frac{1}{2 s+1}\left\langle j_{A}, j_{B}, s-\frac{1}{2}|| f_{N-1}^{B}|| j_{A}, j_{B}-\frac{1}{2}, s\right\rangle_{N-1}^{B}\right\} \text {. }
\end{aligned}
$$

Para calcular os invariantes $<j_{A}, j_{B}, s, k\left\|f_{N}^{B}\right\| j_{A}, j_{B}-\frac{1}{2}, s \pm \frac{1}{2}, l>_{N}^{B}$ na iteração $N$ fase $B$ nós escrevemos o $k$-ésimo e o $l$-ésimo autovetores em termos dos vetores de base. Então, o cálculo involve os invariantes entre vetores de base. Temos quatro tipos desses invariantes (notação: 
$\left.\left\langle j_{A}, j_{B}, s, \mathbf{t i p o}\left\|f_{N-1}^{B}\right\| j_{A}, j_{B}-\frac{1}{2}, s \pm \frac{1}{2}, \mathbf{t i p o}^{\prime}\right\rangle_{N-1}^{B}\right)$ e são eles

$$
\begin{aligned}
\left\langle j_{A}, j_{B}, s, \mathbf{1}\left\|f_{N}^{B}\right\| j_{A}, j_{B}-\frac{1}{2}, s-\frac{1}{2}, \mathbf{3}\right\rangle_{N}^{B} & =(-1)^{N} \sqrt{\frac{2 s}{2 s+1}} \\
\left\langle j_{A}, j_{B}, s, \mathbf{2}\left\|f_{N}^{B}\right\| j_{A}, j_{B}-\frac{1}{2}, s-\frac{1}{2}, \boldsymbol{4}\right\rangle_{N}^{B} & =\sqrt{\frac{2 j_{B}}{2 j_{B}+1}} \\
\left\langle j_{A}, j_{B}, s, \mathbf{1}\left\|f_{N}^{B}\right\| j_{A}, j_{B}-\frac{1}{2}, s+\frac{1}{2}, \mathbf{2}\right\rangle_{N}^{B} & =-(-1)^{N} \sqrt{\frac{2 s+2}{2 s+1}} \\
\left\langle j_{A}, j_{B}, s, \mathbf{3}\left\|f_{N}^{B}\right\| j_{A}, j_{B}-\frac{1}{2}, s+\frac{1}{2}, \mathbf{4}\right\rangle_{N}^{B} & =\sqrt{\frac{2 j_{B}}{2 j_{B}+1}} .
\end{aligned}
$$

De posse dos invariantes usamos as Eqs. (5.29) e (5.34) para montar os Hamiltonianos $H_{N}^{B}$ e $H_{N}^{A}$, respectivamente. Uma vez diagonalizado $H_{N}^{B}$, temos o espectro de energia, rotulado por $j_{A}, j_{B}$ e $s$, e computamos as propriedades termodinâmicas.

\subsection{Termodinâmica}

Calculamos a suscetibilidade magnética e o calor específico para o modelo de Kondo de Dois Canais, para o caso com isotropia de troca (isto é, $J_{x}=J_{y}=J_{z}$ ), mas tanto para o modelo simétrico, $J_{1}=J_{2}$, quanto para o assimétrico, $J_{1} \neq J_{2}$.

Para tal, usamos o procedimento do GRN em duas fases conforme explicitado acima, usando também o parâmetro de discretização adicional z como artifício para eliminar as oscilações espúrias inerentes ao GRN. Para a suscetibilidade, basta em geral que façamos média entre duas curvas com z's diferindo de 0,5 [27]. Já para o calor específico do $\mathrm{MK} 2 \mathrm{C}$ simétrico (com acoplamentos iguais), média com oito valores de $z$ não foram suficientes. Foi então que começamos a repensar nas oscilações espúrias e observamos alternativas à volta ao contínuo da banda de condução que podem ser mais interessantes que a usual média sobre $z$. 


\subsubsection{Método da intersecção}

O método tradicional de tomar médias entre curvas de diferentes valores de $z$ nem sempre dá resultados satisfatórios, como já discutimos anteriormente. Desenvolvemos ainda um outro artifício para driblar esta dificuldade baseado no fato de que, pelo menos a princípio, curvas com $z$ 's diferindo de 0.5 interceptam-se em pontos sobre a curva legítima. Em nossos testes, tais instersecções foram as que se mantiveram mais fiéis dentre todos os pontos gerados pelas médias daquelas curvas. Felizmente, a aparente arbitrariedade deste procedimento desvanece-se quando consideramos que o método tradicional o legitima, pois o ponto médio entre dois pontos coincidentes, coincide com esses.

Como exemplo, observemos a Fig. (5.1). Lá, plotamos duas curvas do calor específico para o

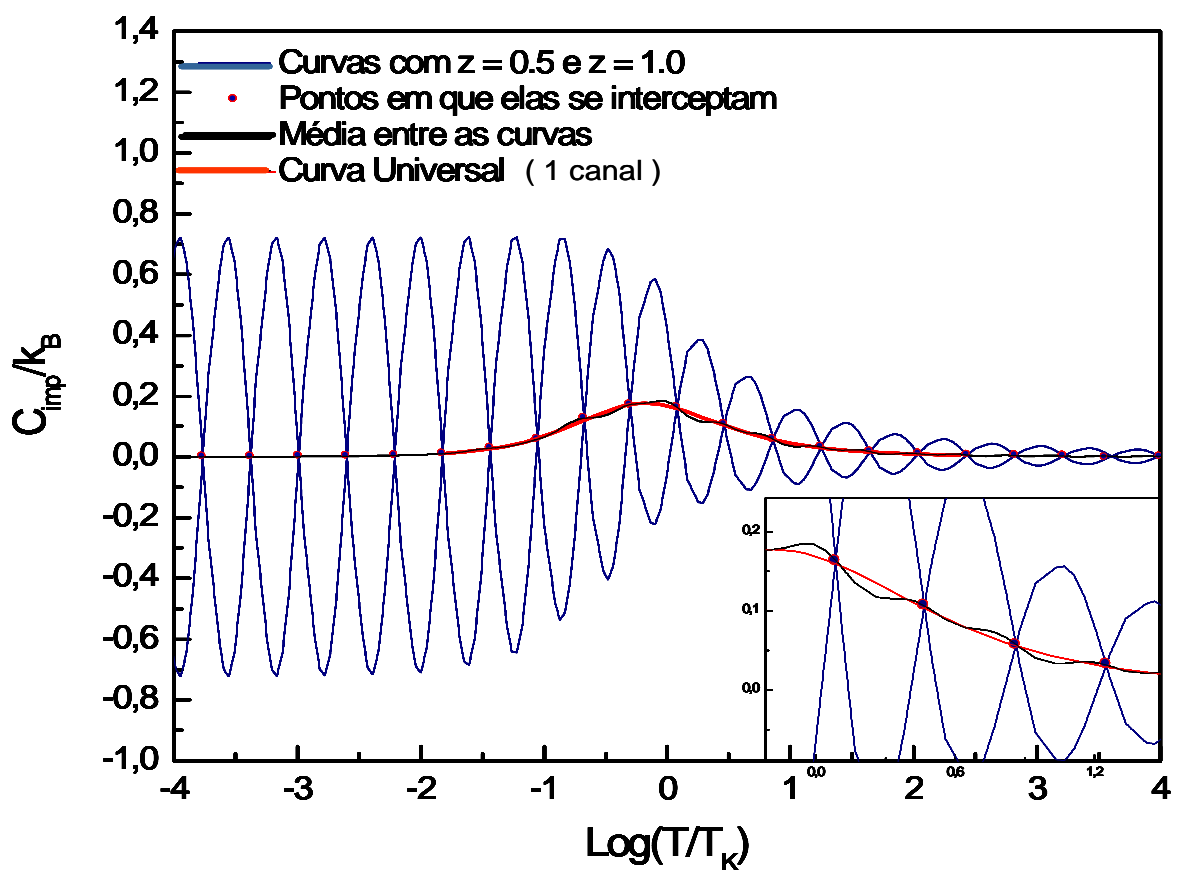

Figura 5.1: Comparação entre a média de duas curvas de calor específico com dois z's diferindo de 0.5 e a curva universal mostrando que os pontos de intersecção pertencem à curva desejada.

MK2C assimétrico de parâmetros $z$ diferindo de 0.5 (curvas azuis). Em preto, está o resultado obtido 
pela simples média entre as curvas. Em vermelho, temos a curva universal para o Modelo de Kondo de Um Canal (MK1C) para fins de comparação. No MK2C assimétrico podemos definir uma temperatura de Kondo associada a cada um dos acopalhamentos. À medida que a tempertura do sistema abaixa, o canal cujo acoplamento tem associado a si temperatura de Kondo mais elevada vai ser o responsável pela blindagem do spin da impureza, restando aos elétrons do outro canal tão somente espalhar na nuvem de singleto que se forma. Por isso, podemos comparar os resultados do MK2C assimétrico com a curva universal para o MK1C. Vemos que os pontos de intersecção concordam muito bem com a curva universal, ao passo que a curva em preto apresenta distorções devidas aos outros pontos que a compõem (veja o trecho ampliado do gráfico).

No exemplo anterior, os pontos das curvas foram unidos por segmentos de reta (para guiar os olhos). Porém, os resultados do GRN são, obviamente, conjuntos discretos de dados. Como geralmente os pontos onde as curvas se interceptariam não pertencem ao conjunto dos que as compõem, fazemos uma interpolação linear de cada uma das curvas nos cruzamentos e determinamos a intersecção entre essas retas interpoladas, como ilustra a Fig. 5.2. Na figura, consideramos uma função discreta que a cada $x_{i}$ relaciona um $y_{i}$ definindo cada um dos pontos $\left(x_{i}, y_{i}\right)$ que determinam a curva. Os sobrescritos $V$ e $A$ rotulam as coordenadas dos pontos vermelhos e azuis, respectivamente. O algorítmo utilizado por nós interpola linearmente todos os pares de pontos das curvas e determina os pontos de intersecção. A reta definida pelos pontos $\left(x_{i}, y_{i}^{V}\right)$ e $\left(x_{i+1}, y_{i+1}^{V}\right)$ intercepta a definida por $\left(x_{i}, y_{i}^{A}\right)$ e $\left(x_{i+1}, y_{i+1}^{A}\right)$ no ponto $\left(X_{i}, Y_{i}\right)$. Se $x_{i}<X_{i}<x_{i+1}$, então $\left(X_{i}, Y_{i}\right)$ é a intersecção que nos interessa.

A seguir, falaremos do procedimento da derivada. Na verdade, não é um método de suavização propriamente dito, mas um procedimento. As curvas da entropia das quais são extraídas as do calor específico através da derivada logarítmica já devem estar tão livres quanto possível das oscilações. E isso pode ser feito bastante eficientemente pelo método da intersecção. Uma vez suavizada desta maneira, podemos obter eficientemente as curvas do calor específico através do procedimento que 


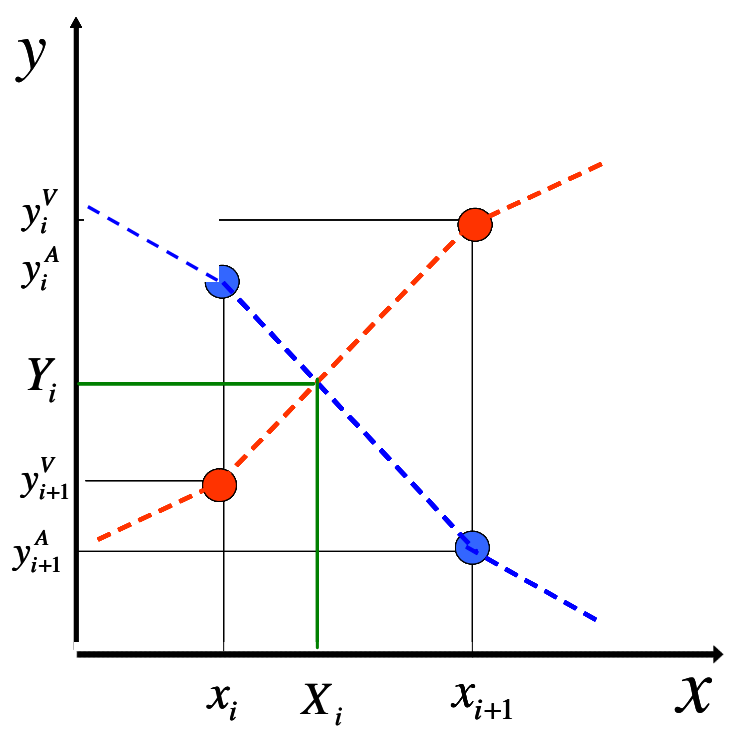

Figura 5.2: Interpolação linear utilizada no método da intersecção. Os pontos são resultados do GRN, enquanto $X_{i}$ e $Y_{i}$ são as coordenadas do ponto de intersecção. Os pontos azuis são para um determinado $z$, enquanto os vermelhos são para outro $z$ diferindo de 0,5 em relação ao dos pontos azuis.

descrevemos abaixo. De fato, as curvas aqui apresentadas foram suavizadas desta maneira.

\subsubsection{Procedimento da derivada}

Quando percebemos que as curvas para a entropia eram tão bem comportadas quanto as da suscetibilidade, passamos a nos preocupar menos com as do calor específico. Primeiro, por causa das oscilações persistentes cujos remédios encontrados são de alcance limitado para o calor específico. O segundo motivo é que, em muitos casos, a entropia supera o calor específico quando se deseja analisar os pontos fixos do modelo. Não obstante isso, gostaríamos de apresentar bons gráficos desta propriedade aproveitando os da entropia. Desta chegamos àquela através da derivada logarítmica. Isto porque a entropia $S$ relaciona-se com o calor específico $C$ através das seguintes equações:

$$
\begin{aligned}
S & =\int \frac{C}{T} d T=\int C d(\ln T), \\
C & =\frac{d S}{d(\ln T)} .
\end{aligned}
$$


As curvas da entropia apresentadas na seção seguinte foram primeiro suavizadas pelo método da intersecção e a partir delas extraídas as curvas do calor específico através do procedimento da derivada.

\subsection{Resultados para o MK2C}

Nesta seção, apresentamos os resultados para o MK2C com campo nulo. Obtivemos curvas da entropia tanto para o caso simétrico quanto assimétrico e curvas correspondentes do calor específico sendo estas últimas obtidas através de derivadas numéricas suavizadas das primeiras.

Dissemos acima que vamos mostrar as curvas para os casos simétrico e assimétrico. Sabemos que se os acoplamentos são iguais, a entropia tem ponto fixo $\ln \sqrt{2}$ e que se um dos acoplamentos é nulo, a entropia comporta-se como MK1C [7, 29]. Então nos perguntamos: e se as acoplamentos forem quase iguais?

A Fig. (5.3) nos ajuda a responder a esta pergunta. Vejamos que, para $J_{1}=0.07999 D$ e $J_{2}=0.08 D$, temos dois graus de liberdade (entendam-se por graus de liberdade a quantidade $e^{\frac{S_{i m p}}{k_{B}}}$ ) em altas temperaturas. A temperaturas mais baixas, $e^{\frac{S_{i m p}}{k_{B}}}$ comporta-se como no MK2C simétrico convergindo para o valor $\sqrt{2}$. Contudo, passa a se comportar como se fosse do caso assimétrico a temperaturas ainda mais baixas ao convergir para o valor 1 (que corresponde à formação do singleto de Kondo). Observemos ainda que mantendo-se $J_{2}=0.08 D$ e diminuindo-se $J_{1}$, faz com que $e^{\frac{S_{i m p}}{k_{B}}}$ se aproxime do MK1C. Intuitivamente, entendemos este comportamento em termos de escalas de energia: a temperaturas mais elevadas as flutuações térmicas são tais que diferenças suficientemente pequenas entre as energias de acoplamento associadas a $J_{1}$ e $J_{2}$ resultam imperceptíveis naquela faixa de temperatura de modo que tudo se passa como se $J_{1}=J_{2}$. No entanto, em região de temperaturas suficientemente baixas, essa mesma diferença sobrepuja as flutuações térmicas de tal forma que o acoplamento mais forte domine e o spin da impureza seja efetivamente blindado, sinalizando a entrada no regime de Líquido de Fermi. 


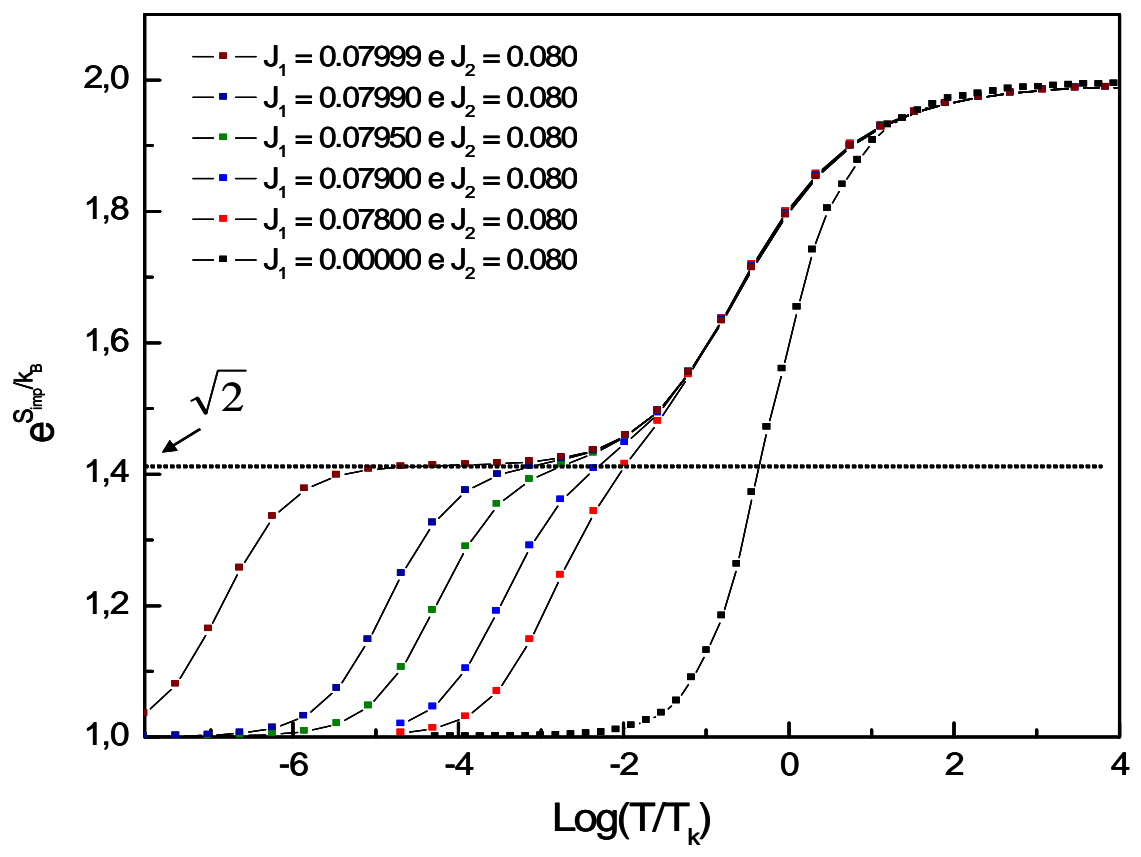

Figura 5.3: Curvas da exponencial da entropia (graus de liberdade) evidenciando situação de "quasesimetria" de canais. O valor de $T_{K}$ é o correspondente a $J=0.08 D$.

Esta migração de comportamento tipicamente associado ao caso simétrico para comportamento tipicamente associado ao caso assimétrico à medida que baixamos a tempertura pode também ser apreciada nas curvas de calor específico.

Acompanhando agora a Fig. (5.4), percebemos que tudo se passa como se o pico correspondente ao MK2C assimétrico com $J_{1}=0$ e $J_{2}=0.08 D$ (equivalente ao MK1C) fosse a superposição de dois picos, sendo que um deles se desloca à esquerda enquanto o outro mantém-se no mesmo lugar à medida que aumentamos o valor de $J_{1}$ próximo à situação em que os acoplamentos são iguais.

Observe o aparecimento de curvas com dois picos: em cada curva, aquele mais à direita é devido à proximidade do sistema ao ponto fixo de 2 canais. Este pico corresponde exatamente, caso não existisse o outro pico, à curva do calor espefícico do $\mathrm{MK} 2 \mathrm{C}$ simétrico, $J_{1}=J_{2}$, correspondendo 
ao mesmo regime observado no patamar na Fig. (5.3). A temperaturas mais baixas, encontramos o outro pico que corresponde ao ponto em que o sistema complementa a blindagem parcial da impureza adquirindo, então, característica de Líquido de Fermi.

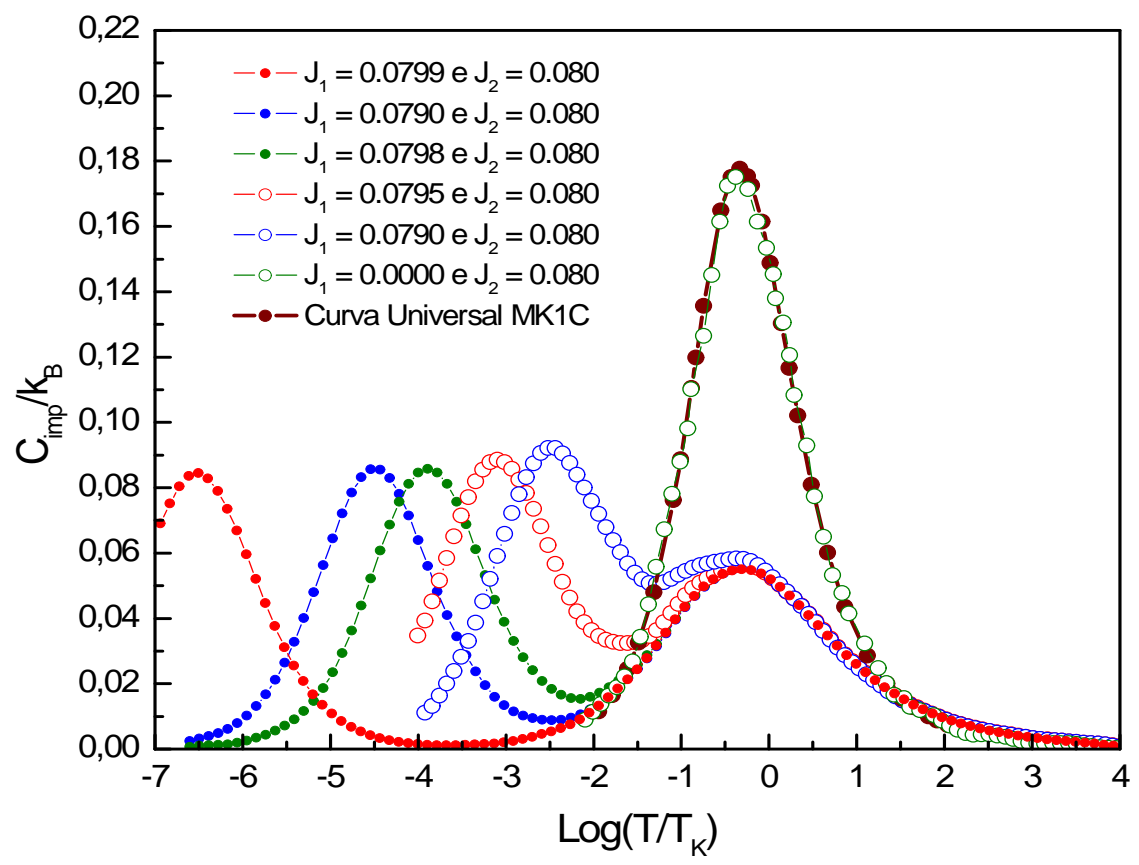

Figura 5.4: Curva para o calor específico para o MK2C mostrando casos em que os valores dos acopalhamentos são muito próximos um do outro.

Observando a Fig. (5.5), vemos que a migração do regime MK2C assimétrico para o MK2C simétrico não se faz de maneira tão efusiva quanto nos casos anteriores.

O conjunto destes resultado nos motiva a explorar este comportamento ambivalente de sistemas de dois canais cujos acoplamentos sejam próximos pela maior riqueza de possibilidades em se comparando com os que usualmente lidamos. Mesmo porque, é possível vislumbrar sistemas em que tais grandezas possam ser variadas facilmente, como o que acontece com poços quânticos de semi-condutores. Nos primórdios do GRN, os únicos sistemas passíveis de serem modelados eram os 


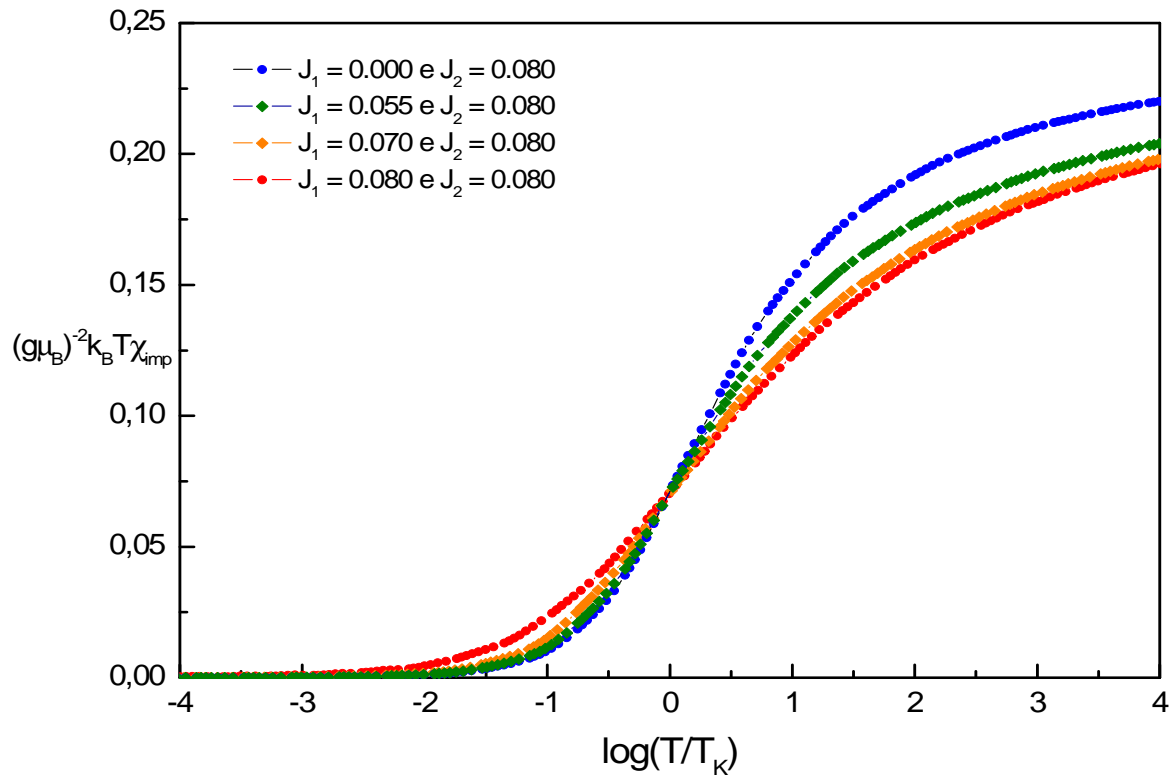

Figura 5.5: Curvas da suscetibilidade magnética para casos em que os valores dos acoplamentos são próximos entre si.

de impureza em metal, estritamente falando, cujos acoplamentos não se poderiam variar num dado material. 


\section{Capítulo 6}

\section{Conclusões}

O crescente desenvolvimento de novos materiais impele a criação de modelos teóricos com tratamentos mais realistas, o que implica, entre outras ações, em melhorias ou generalizações de procedimentos já estabelecidos. O Grupo de Renormalização Numérico [2, 6] sendo não perturbativo mostrou-se muito importante no estudo dos modelos de impureza localizada, com ou sem caráter magnético. Sua aplicação original foi no modelo de Kondo [4] de uma impureza magnética de spin 1/2, uma versão simplificada de um problema real de laboratório, mas que procurava descrever a essência de uma classe de sistemas físicos. Com o tempo, essa técnica passou a ser implementada também para situações em que duas impurezas se correlacionavam entre si e com uma banda de condução [10]. Mais recentemente têm-se tratado modelos em que a banda de condução também apresenta estruturas [25], denominadas canais [7].

Apresentamos neste trabalho duas implementações do Grupo de Renormalização Numérico (GRN). Em uma delas consideramos um campo magnético externo atuando sobre os spins eletrônicos. A banda de condução, embora com apenas um canal, adquire uma assimetria em torno do nível de Fermi. Utilizamos o Modelo de Nível Ressonante para desenvolver o método e assim poder comparar com resultados da literatura (que foram generalizados para serem devidamente comparados). O ponto 
relevante nessa implementação do GRN é a assimetria da banda, que não necessariamente tem sua origem num campo magnético. Por exemplo, impurezas localizadas em sistemas metálicos com banda fora do semipreenchimento podem ser estudadas utilizando-se o GRN desenvolvido aqui. Nessa direção, aplicamos o procedimento ao modelo de Falicov-Kimball [9] para duas impurezas, hibridizadas com uma banda de condução e eletrostaticamente acopladas a esta. Determinamos propriedades termodinâmicas na presença de um campo atuando tanto nas cargas de condução como nas localizadas. Em seguida, tratamos um sistema em que a banda é simétrica, mas ainda dentro do contexto de bandas com estrutura, nós a consideramos constituída de dois tipos de elétrons, rotulados por números quânticos denominados canais [7]. Aqui enfrentamos dificuldades para retornar ao contínuo da banda de condução ao final da implementação do GRN (que discretiza a banda para torná-la numericamente tratável), particularmente para o modelo simétrico (iguais acoplamentos dos canais com a banda) em que o limite de baixas temperaturas é de não-líquido de Fermi. Alternativas foram propostas, mas que necessitam de mais investigações para serem julgadas, particularmente sua eficiência em outros modelos.

Os desenvolvimentos apresentados neste trabalho são frutos de importantes generalizações anteriores feitas em nosso grupo de trabalho [10, 11, 12, 22, 23, 25, 26, 27, 28, 31, 32]. De forma nenhuma esgota as possibilidades de implementações ainda mais realistas, ou simplesmente mais eficientes, dessa poderosa técnica, o GRN. Nem este trabalho explorou todas as possibilidades de aplicações aos modelos estudados. Esperamos, contudo, ter contribuído para futuros trabalhos nessa direção. Em particular, para o modelo de FK uma investigação mais profunda é necessária para se concluir se o regime de líquido de Fermi é dependente do campo aplicado ou não. O relaxamento da aproximação $\epsilon \rightarrow \epsilon_{F}$ feita na densidade de estados $\rho(\epsilon)$ e na variável $S=\sin (k(\epsilon) R) /(k(\epsilon) R)$ também provoca uma assimetria na banda de condução [26]. Como essa assimetria compete com a introduzida aqui, devida ao campo $B$, pode ser estudado seguindo as linhas deste trabalho. Quanto ao modelo de Kondo de 
dois canais, a implementação em duas fases por iteração (anteriormente feita para um modelo com um canal e duas impurezas [10]) prepara o terreno para uma implementação mais custosa computacionalmente quando aplicarmos um campo magnético ao sistema. Isso porque esse campo quebra a simetria partícula-buraco e aí, não podendo ser usada as conservações de isospin, teremos matrizes bem maiores para serem diagonalizadas. Antevemos em decorrência da menor precisão dos cálculos neste caso que as oscilações espúrias terão ainda maiores amplitudes que aquelas que enfrentamos aqui. Os métodos alternativos de retorno ao contínuo aqui apresentados poderão ter suas eficiências testadas. 


\section{Apêndice A - Função Poligama}

A função poligama de ordem $n$ é definida por [33]:

$$
\Psi_{n}(z)=\frac{d^{n+1}}{d z^{n+1}} \ln [\Gamma(z)]
$$

onde $\Gamma(z)$ é a função gama de argumento complexo $z$ definida por

$$
\Gamma(z)=k^{z} \int_{0}^{\infty} t^{z-1} e^{-k t} d t, \quad(\operatorname{Re}(z)>0, \operatorname{Re}(k)>0),
$$

sendo $\operatorname{Re}(z)$ a parte real de $z$. Convenciona-se chamar $\Psi_{0}(z), \Psi_{1}(z), \Psi_{2}(z)$ etc. de função digama, trigama, tetragama etc., respectivamente. É também usual adotar $\Psi(z)$ para a função digama e $\Psi^{\prime}(z)$ para a função trigama. De fato, aqui usamos tal convenção.

Dentre as propriedades das funções poligamas, a que nos foi mais útil foi a chamada fórmula da multiplicação:

$$
\Psi_{n}(m z)=\delta_{n 0} \ln m+\frac{1}{m^{n+1}} \sum_{k=0}^{m-1} \Psi_{n}\left(z+\frac{k}{m}\right),
$$

onde $m$ é um número inteiro maior que zero e $\delta_{n p}$ é a delta de Kronecker. Em particular, para a função trigama temos

$$
\Psi^{\prime}(2 z)=\frac{1}{4}\left[\Psi^{\prime}(z)+\Psi^{\prime}\left(z+\frac{1}{2}\right)\right]
$$




\section{Apêndice B: Quantidades}

\section{termodinâmicas para a banda livre}

As quantidades termodinâmicas que estamos interessados neste trabalho referem-se às diferenças entre as calculadas para o sistema completo, metal mais impureza, e as calculadas para o metal somente. Desta forma, temos a contribuição da impureza à propriedade em estudo. Sendo o metal representado por elétrons não interagentes, a cada iteração o Hamiltoniano de banda livre que os representa é diagonalizado fornecendo as energias de partícula independente. De posse desses autovalores, o tratamento de muitos corpos é feito analiticamente a partir da função de partição:

$$
Z=\operatorname{Tr}\left[e^{-\beta\left(H_{0}-B S_{z}\right)}\right]
$$

onde $H_{0}$ representa a banda livre e $B \equiv g \mu_{B} B_{z} / \hbar$, com $B_{z}$ a componente $z$ do campo aplicado.

A banda de condução é descrita pelo Hamiltoniano quadrático $H_{0}=\sum \epsilon_{n} g_{n \sigma}^{\dagger} g_{n \sigma}$, onde $g_{n \sigma}^{\dagger}$ é o operador que cria uma partícula no nível de energia $\epsilon_{n}$, com componente $z$ do spin igual a $\sigma$. O nível $\left|\mu_{n}\right\rangle$ de partícula independente com energia $\epsilon_{n}$ pode estar vazio, unicamente ocupado ou duplamente 
ocupado. Logo, a função de partição será dada por:

$$
\begin{aligned}
Z & =\sum_{\left\{\mu_{i}\right\}}\left\langle\mu_{1}\left|e^{-\beta\left(H_{0}-B S_{z}\right)}\right| \mu_{1}\right\rangle\left\langle\mu_{2}\left|e^{-\beta\left(H_{0}-B S_{z}\right)}\right| \mu_{2}\right\rangle \ldots\left\langle\mu_{N}\left|e^{-\beta\left(H_{0}-B S_{z}\right)}\right| \mu_{N}\right\rangle \\
& =\prod_{i=1}^{N}\left[1+e^{-2 \beta \varepsilon_{i}}+e^{-\beta\left(\varepsilon_{i}-B / 2\right)}+e^{-\beta\left(\varepsilon_{i}+B / 2\right)}\right] \\
& =\prod_{i=1}^{N}\left[e^{-\beta \varepsilon_{i}}\left(e^{+\beta \varepsilon_{i}}+e^{-\beta \varepsilon_{i}}\right)+e^{-\beta \varepsilon_{i}}\left(e^{\beta B / 2}+e^{-\beta B / 2}\right)\right] \\
& =\prod_{i=1}^{N}\left[2 e^{-\beta \varepsilon_{i}} \cosh \left(\beta \varepsilon_{i}\right)+2 e^{-\beta \varepsilon_{i}} \cosh \left(\frac{\beta B}{2}\right)\right] \\
& =\prod_{i=1}^{N} 2 e^{-\beta \varepsilon_{i}}\left[\cosh \left(\beta \varepsilon_{i}\right)+\cosh \left(\frac{\beta B}{2}\right)\right]
\end{aligned}
$$

que pode ser reescrita como:

$$
Z=\prod_{i=1}^{N} 4 e^{-\beta \varepsilon_{i}} \cosh \left[\frac{\beta}{2}\left(\varepsilon_{i}+\frac{B}{2}\right)\right] \cosh \left[\frac{\beta}{2}\left(\varepsilon_{i}-\frac{B}{2}\right)\right]
$$

Da definição de energia média,

$$
\langle E\rangle \equiv \frac{1}{Z} \operatorname{Tr}\left(H e^{-\beta H}\right)=-\frac{d}{d \beta} \ln Z,
$$

resulta

$$
\langle E\rangle=\sum_{i=1}^{N}\left\{\varepsilon_{i}-\frac{1}{2}\left(\varepsilon_{i}+\frac{B}{2}\right) \tanh \left[\frac{\beta}{2}\left(\varepsilon_{i}+\frac{B}{2}\right)\right]-\frac{1}{2}\left(\varepsilon_{i}-\frac{B}{2}\right) \tanh \left[\frac{\beta}{2}\left(\varepsilon_{i}-\frac{B}{2}\right)\right]\right\}
$$

Com isso, podemos calcular a entropia através de

$$
\frac{S}{k_{B}} \equiv \beta\langle E\rangle+\ln Z
$$

resultando em

$$
\begin{aligned}
\frac{S}{k_{B}} & =-\beta \sum_{i=1}^{N}\left\{\frac{1}{2}\left(\varepsilon_{i}+\frac{B}{2}\right) \tanh \left[\frac{\beta}{2}\left(\varepsilon_{i}+\frac{B}{2}\right)\right]+\frac{1}{2}\left(\varepsilon_{i}-\frac{B}{2}\right) \tanh \left[\frac{\beta}{2}\left(\varepsilon_{i}-\frac{B}{2}\right)\right]\right\} \\
& +\sum_{i=1}^{N}\left\{\ln 4+\ln \left(\cosh \left[\frac{\beta}{2}\left(\varepsilon_{i}+\frac{B}{2}\right)\right]\right)+\ln \left(\cosh \left[\frac{\beta}{2}\left(\varepsilon_{i}-\frac{B}{2}\right)\right]\right)\right\} .
\end{aligned}
$$


Para o calor específico, de

$$
\frac{C}{k_{B}} \equiv-\beta^{2} \frac{d\langle E\rangle}{d \beta}
$$

vem que

$$
\frac{C}{k_{B}}=\frac{\beta^{2}}{4} \sum_{i=1}^{N}\left(\left(\varepsilon_{i}+\frac{B}{2}\right)^{2} \operatorname{sech}^{2}\left[\frac{\beta}{2}\left(\varepsilon_{i}+\frac{B}{2}\right)\right]+\left(\varepsilon_{i}-\frac{B}{2}\right)^{2} \operatorname{sech}^{2}\left[\frac{\beta}{2}\left(\varepsilon_{i}-\frac{B}{2}\right)\right]\right) .
$$

Para a magnetização, da definição

$$
\frac{M}{g \mu_{B}} \equiv \frac{1}{Z} \operatorname{Tr}\left(S_{z} e^{-\beta\left(H_{0}-B S_{z}\right)}\right)
$$

obtemos

$$
\begin{aligned}
\frac{M}{g \mu_{B}} & =\frac{1}{\beta} \frac{\partial}{\partial B} \ln Z \\
& =\frac{1}{4} \sum_{i=1}^{N}\left\{\tanh \left[\frac{\beta}{2}\left(\varepsilon_{i}+\frac{B}{2}\right)\right]-\tanh \left[\frac{\beta}{2}\left(\varepsilon_{i}-\frac{B}{2}\right)\right]\right\} .
\end{aligned}
$$

Para conseguirmos a suscetibilidade, basta derivarmos $M$ mais uma vez em $B$ :

$$
\frac{k_{B} T \chi}{\left(g \mu_{B}\right)^{2}}=\frac{\beta}{8} \sum_{i=1}^{N}\left(\operatorname{sech}^{2}\left[\frac{\beta}{2}\left(\varepsilon_{i}+\frac{B}{2}\right)\right]+\operatorname{sech}^{2}\left[\frac{\beta}{2}\left(\varepsilon_{i}-\frac{B}{2}\right)\right]\right) .
$$

Temos, então, as equações para a entropia, calor específico e suscetibilidade magnética para a banda livre. Para o modelo Kondo de dois canais, multiplicamos cada expressão acima por dois, referente à contribuição independente de cada canal. 


\section{Apêndice C - Os operadores $f_{n, \sigma, \alpha}$ são}

\section{tensores}

Vamos mostrar que os operadores $f_{n, \sigma, \alpha}$ e $f_{n, \sigma, \alpha}^{\dagger}$ são as componentes $\pm 1 / 2$ de um tensor. Isso justifica o uso do teorema de Wigner-Eckart. Seguindo Fetter \& Walecka [34], $T(k, q)$ é a componente $q$ de um tensor de ordem $k$ se

$$
\left[j_{z}, T(k, q)\right]=q T(k, q)
$$

e

$$
\left[j_{ \pm}, T(k, q)\right]=A(k, \mp q) T(k, q \pm 1)
$$

Usando a Eq. (5.9), nós achamos que

$$
\begin{aligned}
& {\left[j_{z}^{\alpha}, f_{n, \sigma, \alpha}^{\dagger}\right]=f_{n, \sigma, \alpha}^{\dagger},} \\
& {\left[j_{z}^{\alpha}, f_{n, \sigma, \alpha}\right]=-f_{n, \sigma, \alpha}}
\end{aligned}
$$

e

$$
\left[j_{-}^{\alpha}, f_{n, \sigma, \alpha}^{\dagger}\right]=(-1)^{n}(2 \sigma) f_{n,-\sigma, \alpha}
$$


os quais definem $f_{n, \sigma, \alpha}^{\dagger}$ como a componente $q=1 / 2$ de um tensor e $(-1)^{n}(2 \sigma) f_{n,-\sigma, \alpha}$ como a componente $q=-1 / 2$. Portanto, nós podemos formar o seguinte tensor:

$$
\left(\begin{array}{c}
f_{n, \sigma, \alpha}^{\dagger} \\
(-1)^{n}(2 \sigma) f_{n,-\sigma, \alpha}
\end{array}\right)
$$

O teorema de Wigner-Eckart pode então ser estabelecido como [34]:

$$
\left\langle j^{\prime}, m^{\prime}|T(k, q)| j, m\right\rangle=\left\langle j, m ; k, q \mid j^{\prime}, m^{\prime}\right\rangle\left\langle j^{\prime}|| T_{K} \| j\right\rangle
$$

onde o primeiro elemento de matriz à direita é o coeficiente de Clebsh-Gordon da adição do momento angular $k$, componente $q$, com momento $j$, componente $m$, para obter o momento $j^{\prime}$, componente $m^{\prime}$. Multiplicando está o elemento de matriz invarinte (isto é, independente da componente $z$ do tensor). Em nosso caso, para $q=1 / 2$,

$$
\begin{aligned}
& \left\langle\quad j_{1}^{\prime}, j_{1}^{\prime} z, j_{2}^{\prime}, j_{2}^{\prime}{ }^{z}, s^{\prime}, s^{\prime} z\left|f_{n, \sigma, \alpha}^{\dagger}\right| j_{1}, j_{1}^{z}, j_{2}, j_{2}^{z}, s, s_{z}\right\rangle= \\
& \left\langle\quad j_{\alpha}, j_{\alpha}^{z} ; \frac{1}{2}, \frac{1}{2} \mid j_{\alpha}^{\prime}, j_{\alpha}^{\prime}\right\rangle\left\langle s, s^{z} ; \frac{1}{2}, \sigma \mid s^{\prime}, s^{\prime} z\right\rangle\left\langle j_{1}^{\prime}, J_{2}^{\prime}, s^{\prime}\|\alpha\| j_{1}, j_{2}, s\right\rangle
\end{aligned}
$$

e, $\operatorname{para} q=-1 / 2$

$$
\begin{aligned}
& \left\langle\quad j_{1}^{\prime}, j_{1}^{\prime}, j_{2}^{\prime}, j_{2}^{\prime}, s^{\prime}, s^{\prime}{ }^{z}\left|(-1)^{n}(-2 \sigma) f_{n, \sigma, \alpha}\right| j_{1}, j_{1}^{z}, j_{2}, j_{2}^{z}, s, s_{z}\right\rangle= \\
& \left\langle\quad j_{\alpha}, j_{\alpha}^{z} ; \frac{1}{2},-\frac{1}{2} \mid j_{\alpha}^{\prime}, j_{\alpha}^{\prime}\right\rangle\left\langle s, s^{z} ; \frac{1}{2},-\sigma \mid s^{\prime}, s^{\prime}{ }^{z}\right\rangle\left\langle j_{1}^{\prime}, J_{2}^{\prime}, s^{\prime}\|\alpha\| j_{1}, j_{2}, s\right\rangle .
\end{aligned}
$$




\title{
Apêndice D: Bloco Diagonalização de
}

\author{
$H_{0, B}$
}

Para construir a base de estados de $H_{0, B}$ nós devemos combinar um estado de spin local $S_{I}$ com estados ou vazio, ou com um $(\uparrow$ ou $\downarrow)$, ou com dois $(\uparrow \downarrow)$ elétrons de condução, e isso de cada canal; resulta, então, $32(=2 \times 4 \times 4)$ estados de base rotulados por $\left|J_{1}, J_{1}^{z}, J_{2}, J_{2}^{z}, S, S_{z}\right\rangle$. No entanto, devido à conservação de $I_{1}^{2}, I_{2}^{2}, I_{1}^{z}, I_{2}^{z}, S^{2}$ e $S_{z}$ nós não precisamos lidar com todos esses estados: nós mantemos apenas aqueles com $J_{1}^{z}=J_{1}, J_{2}^{z}=J_{2}$ e $s_{z}=s$.

Isso reduz a oito os estados de base:

$$
\begin{aligned}
|1\rangle_{0}^{B} & =\left|0,0, \quad 0,0, \quad \frac{1}{2}, \frac{1}{2}\right\rangle=\frac{1}{\sqrt{6}}(2|\downarrow, \uparrow, \uparrow\rangle-|\uparrow, \uparrow, \downarrow\rangle-|\uparrow, \downarrow, \uparrow\rangle) \\
|2\rangle_{0}^{B} & =\left|0,0, \quad 0,0, \quad \frac{1}{2}, \frac{1}{2}\right\rangle=\frac{1}{\sqrt{2}}(|\uparrow, \uparrow, \downarrow\rangle-|\uparrow, \downarrow, \uparrow\rangle) \\
|3\rangle_{0}^{B} & =\left|0,0, \quad 0,0, \frac{3}{2}, \frac{3}{2}\right\rangle=|\uparrow, \uparrow, \uparrow\rangle \\
|4\rangle_{0}^{B} & =\left|0,0, \quad \frac{1}{2}, \frac{1}{2}, \quad 0,0\right\rangle=\frac{1}{\sqrt{2}}(|\uparrow, \uparrow \downarrow, \downarrow\rangle-|\downarrow, \uparrow \downarrow, \uparrow\rangle)
\end{aligned}
$$




$$
\begin{aligned}
|5\rangle_{0}^{B} & =\left|0,0, \quad \frac{1}{2}, \frac{1}{2}, \quad 1,1\right\rangle=|\uparrow, \uparrow \downarrow, \uparrow\rangle \\
|6\rangle_{0}^{B} & =\left|\frac{1}{2}, \frac{1}{2}, \quad 0,0, \quad 0,0\right\rangle=\frac{1}{\sqrt{2}}(|\uparrow \downarrow, \uparrow, \downarrow\rangle-|\uparrow \downarrow, \downarrow, \uparrow\rangle) \\
|7\rangle_{0}^{B} & =\left|\frac{1}{2}, \frac{1}{2}, \quad 0,0, \quad 1,1\right\rangle=|\uparrow \downarrow, \uparrow, \uparrow\rangle \\
|8\rangle_{0}^{B} & =\left|\frac{1}{2}, \frac{1}{2}, \quad \frac{1}{2}, \frac{1}{2}, \quad \frac{1}{2}, \frac{1}{2}\right\rangle=|\uparrow \downarrow, \uparrow \downarrow, \uparrow\rangle .
\end{aligned}
$$

No processo iterativo do GRN, todos os auto-estados são levados em conta pelo uso do teorema de Wigner-Eckart, aplicável aqui uma vez que os operadores $f_{n, \sigma, \alpha}$ são tensoriais (ver Apêndice C). Desse modo, cada auto-estado é $\left(2 J_{1}+1\right) \times\left(2 J_{2}+1\right) \times(2 S+1)$ degenerado.

Os blocos diagonais de $H_{0, B}$ são:

$$
\begin{aligned}
& \left|0,0 ; 0,0 ; \frac{1}{2}, \frac{1}{2}\right\rangle \rightarrow H_{0 B}^{00 \frac{1}{2}}=\Lambda^{-1 / 2}\left(\begin{array}{cc}
\frac{J_{2}-2 J_{1}}{4} & \frac{\sqrt{3}}{4} J_{1} \\
\frac{\sqrt{3}}{4} J_{1} & -\frac{3 J_{2}}{4}
\end{array}\right) \\
& \left|0,0 ; 0,0 ; \frac{3}{2}, \frac{3}{2}\right\rangle \rightarrow H_{0 B}^{00 \frac{3}{2}}=\Lambda^{-1 / 2}\left(\frac{J_{1}+J_{2}}{4}\right) \\
& \left|0,0 ; \frac{1}{2}, \frac{1}{2} ; 0,0\right\rangle \rightarrow H_{0 B}^{0 \frac{1}{2} 0}=\Lambda^{-1 / 2}\left(-\frac{3 J_{1}}{4}\right) \\
& \left|0,0 ; \frac{1}{2}, \frac{1}{2} ; 1,1\right\rangle \rightarrow H_{0 B}^{0 \frac{1}{2} 1}=\Lambda^{-1 / 2}\left(\frac{J_{1}}{4}\right) \\
& \left|\frac{1}{2}, \frac{1}{2} ; 0,0 ; 0,0\right\rangle \rightarrow H_{0 B}^{\frac{1}{2} 00}=\Lambda^{-1 / 2}\left(-\frac{3 J_{2}}{4}\right) . \\
& \left|\frac{1}{2}, \frac{1}{2} ; 0,0 ; 1,1\right\rangle \rightarrow H_{0 B}^{\frac{1}{2} 01}=\Lambda^{-1 / 2}\left(\frac{J_{2}}{4}\right) . \\
& \left|\frac{1}{2}, \frac{1}{2} ; \frac{1}{2}, \frac{1}{2} ; \frac{1}{2}, \frac{1}{2}\right\rangle \rightarrow H_{0 B}^{\frac{1}{2} \frac{1}{2} \frac{1}{2}}=-\Lambda^{-1 / 2}\left(\begin{array}{l}
0 \\
0
\end{array}\right.
\end{aligned}
$$


Note a simetria de canal na troca $J_{1} \rightleftharpoons J_{2}$.

Para completar a fase- $B$ na iteração $N=0$ nós calculamos os elementos invariantes a serem usados em $N=1$, fase $A$. Eles são definidos pelas relações

$$
\begin{aligned}
& \left\langle j_{A}^{\prime}, j_{A}^{\prime}, j_{B}, j_{B}^{z}, s^{\prime}, s^{\prime z}\left|f_{0, \sigma, A}^{\dagger}\right| j_{A}, j_{A}^{z}, j_{B}, j_{B}^{z}, s, s^{z}\right\rangle_{N=0}^{B} \\
= & \left\langle j_{A}, j_{A}^{z} ; \frac{1}{2}, \frac{1}{2} \mid j_{A}^{\prime}, j_{A}^{\prime}\right\rangle\left\langle s, s^{z} ; \frac{1}{2}, \sigma \mid s^{\prime}, s^{\prime}{ }^{z}\right\rangle\left\langle j_{A}^{\prime}, j_{B}, s^{\prime}\left\|f^{A}\right\| j_{A}, j_{B}, s\right\rangle_{0}^{B} .
\end{aligned}
$$

Exemplo:

$$
\begin{aligned}
& \left\langle\frac{1}{2}, 0,0,1\left|f_{0, \downarrow, A}^{\dagger}\right| 0,0, \frac{1}{2}, i\right\rangle_{0}^{B}=\langle 6| f_{0, \downarrow, A}^{\dagger}\left(\text { vet }_{1, i}|15\rangle+\text { vet }_{2, i}|2\rangle\right) \\
= & \frac{1}{\sqrt{2}}\langle\uparrow \downarrow, \uparrow, \downarrow|-\left\langle\uparrow \downarrow, \downarrow, \uparrow\left|f_{0, \downarrow, A}^{\dagger}\right|\left|\operatorname { v e t } _ { 1 , i } \left(\frac{1}{\sqrt{6}}(2|\downarrow, \uparrow, \uparrow\rangle-|\uparrow, \uparrow, \downarrow\rangle-|\uparrow, \downarrow, \uparrow\rangle)\right.\right.\right. \\
+ & \operatorname{vet}_{2, i}\left(\frac{1}{\sqrt{2}}(|\uparrow, \uparrow, \downarrow\rangle-|\uparrow, \downarrow, \uparrow\rangle)\right] \\
= & \frac{1}{\sqrt{2}}\left(\frac{\text { vet }_{1, i}}{\sqrt{6}}-\frac{\text { vet }_{2, i}}{\sqrt{2}}-\frac{\text { vet }_{1, i}}{\sqrt{6}}-\frac{\text { vet }_{2, i}}{\sqrt{2}}\right)=\text {-vet }_{2, i},
\end{aligned}
$$

onde $v_{e} t_{i, j}$ é a $i$-ésima linha do $j$-ésimo autovetor do subespaço $|0,0,1 / 2\rangle$.

Mas esse elemento de matriz pode também ser calculado da seguinte maneira:

$$
\begin{aligned}
& \left\langle\frac{1}{2}, 0,0,1\left|f_{0, \downarrow}^{\dagger}\right| 0,0, \frac{1}{2}, i\right\rangle_{0}^{B}= \\
& =\left\langle 0,0 ; \frac{1}{2}, \frac{1}{2} \mid \frac{1}{2}, \frac{1}{2}\right\rangle\left\langle\frac{1}{2}, \frac{1}{2} ; \frac{1}{2},-\frac{1}{2} \mid 0,0\right\rangle\left\langle\frac{1}{2}, 0,0,1|| f^{A}|| 0,0, \frac{1}{2}, i\right\rangle_{0}^{B}= \\
& =1 \sqrt{\frac{1 / 2+1 / 2}{2}}=\frac{1}{\sqrt{2}}\left\langle\frac{1}{2}, 0,0,1|| f^{A}|| 0,0, \frac{1}{2}, i\right\rangle .
\end{aligned}
$$

Portanto, igualando, temos

$$
\left\langle\frac{1}{2}, 0,0,1\left\|f_{0}^{A}\right\| 0,0, \frac{1}{2}, i\right\rangle_{0}^{B}=-\sqrt{2} \text { vet }_{2, i} .
$$


Assim, os invariantes em $N=0$, fase $B$, são: (notação: $\left\langle I_{1}^{\prime}, I_{2}, s^{\prime}, j\|A\| I_{1}, I_{2}, s, i\right\rangle$ ):

$$
\begin{aligned}
& 1-\left\langle\frac{1}{2}, 0,0,1\left\|f^{A}\right\| 0,0, \frac{1}{2}, 1\right\rangle_{0}^{B}=-\sqrt{2} \operatorname{vet}(2,1) \\
& 2-\left\langle\frac{1}{2}, 0,0,1\left\|f^{A}\right\| 0,0, \frac{1}{2}, 2\right\rangle_{0}^{B}=-\sqrt{2} \operatorname{vet}(2,2) \\
& 3-\left\langle\frac{1}{2}, 0,1,1\left\|f^{A}\right\| 0,0, \frac{1}{2}, 1\right\rangle_{0}^{B}=\sqrt{2 / 3} \operatorname{vet}(1,1) \\
& 4-\left\langle\frac{1}{2}, 0,1,1\left\|f^{A}\right\| 0,0, \frac{1}{2}, 2\right\rangle_{0}^{B}=\sqrt{2 / 3} \operatorname{vet}(1,2) \\
& 5-\left\langle\frac{1}{2}, 0,1,1\left\|f^{A}\right\| 0,0, \frac{3}{2}, 1\right\rangle_{0}^{B}=-2 / \sqrt{3} \\
& 6-\left\langle\frac{1}{2}, \frac{1}{2}, \frac{1}{2}, 1\left\|f^{A}\right\| 0, \frac{1}{2}, 0,1\right\rangle_{0}^{B}=-1 / \sqrt{2} \\
& 7-\left\langle\frac{1}{2}, \frac{1}{2}, \frac{1}{2}, 1\left\|f^{A}\right\| 0, \frac{1}{2}, 1,1\right\rangle_{0}^{B}=-\sqrt{3 / 2} .
\end{aligned}
$$

Com esses estados de $H_{0, B}$ e invariantes construímos a base de estados de $H_{1, A}$; ela terá 25 estados, com o maior bloco, $5 \times 5$. Sem as conservações utilizadas teríamos uma matriz de ordem 512 . 


\section{Referências Bibliográficas}

[1] Jarillo-Herrero, P.; Kong, J; Zant, H. S.J.; Dekker, C.; Kouwenhoven, L.P. e Franceschi, S., Nature 434, 484 (2005).

[2] Wilson, K. G. Rev. Modern Phys. 47, 773 (1975).

[3] Meissner,W. ;Voigt, B. Ann. Physik 7, 761 (1930).

[4] Kondo, J; J. Prog. Theor. Phys.,32, 37 (1964).

[5] Krishna-Murthy, H.R.J.; Wilkins, W. e Wilson, K. G. Phys. Rev. B 21, 1003 (1980)

[6] Anderson, P. W. Phys. Rev. Lett., 18, 1049 (1967).

[7] Nozières, P. H. e Blandin, A. J. Physique 41, 193 (1980).

[8] Yoshida, M; Whitaker, M. A. e Oliveira, L. N., Phys. Rev. B 41, 9403 (1990).

[9] Falicov, L. M.; e Kimball, J.C Phys. Rev. Lett. 22, 997 (1969).

[10] Paula, C. A. Densidade espectral para o modelo de Anderson de duas impurezas. Tese de doutorado apresentada ao Instituto de Física de São Carlos - USP (1998)

[11] L. N. Oliveira, Renormalization group approach to the $x$-ray absorption problem and application to the Vigman-Finkelshtein model for magnetic impurities in metal. Tese de doutorado apresentada na Faculty of the Graduate School, Cornell University (1981). 
[12] Lima, W.L.C., Tese de Doutorado apresentada ao Instituto de Física de São Carlos - USP (1998)

[13] Schotte, K. D.; Schotte, U., Phys. Rev. Lett. 55A, 38 (1975).

[14] Ramirez, R; L. M. Falicov e J. C. Kimball, Phys. Rev. B 2, 3383 (1970).

[15] G. Czycholl, Phys. Rev. B 59, 2642 (1999).

[16] Schiller, A.; Phys. Rev. B 60, 15660 (1999).

[17] Chung, Woonki e Fredericks, J.K. Phys. Rev. Letters 84, 2461 (2000).

[18] Gruber, C. ; Ueltschi, D. e Jedrzejewski, J. J. Stat. Phys. 76, 125 (1994).

[19] Macedo, C. A.; Azevedo, L. G. e Souza, A. M. C., Phys. Rev. B 64, 184441 (2001).

[20] Dongen, P. G. J., Phys. Rev. B 45, 2267 (1992).

[21] Farkasovsky, P. Phys. Rev. B 54, 7865 (1996).

[22] Líbero, V.L. e Oliveira, L.N. Phys. Rev. Lett. 16, 2042 (1990).

[23] Silva, J.B.; Lima, W. L. C.; Oliveira, W.C.; Mello, J. L. N.; Oliveira, L. N. Wilkins, J.W. Phys. Rev. Lett. 76, 275 (1996).

[24] Jones, B. A.; Varma, C. M. e Wilkins, J. W. Phys. Rev. Lett., 61, 125 (1988).

[25] Ferreira, J. V. B. e Líbero V. L. , Phys. Rev. B 61, 10615 (2000).

[26] Ramos, L. R. Propriedades termodinâmicas dos modelo de Falicov-Kimball de duas impurezas sem spin, Tese de doutorado apresentada ao Instituto de Física de São Carlos (2002).

[27] Costa, S.C; Paula, C. A.; Líbero,V. L. e Oliveira, L. N., Phys. Rev. B 55, 30 (1997).

[28] Oliveira,W. C.; e Oliveira, L. N., Phys. Rev. B 49, 11986 (1994). 
[29] Pang, H.B. e Cox, D.L., Phys. Rev. B 44, 8454 (1991).

[30] Tinkham, M; Group Theory and Quantum Mechanics, Nova Iorque, Mcgraw-Hill (1964).

[31] CAMPO JR., V.L., O Grupo de Renormalização Numérico e o Problema de Duas Impurezas, Tese de doutorado apresentada ao Instituto de Física de São Carlos - USP (2004).

[32] Ferreira, J.V.B.; Modelo de Anderson de Dois Canais, Tese de doutorado apresesentada ao Instituto de Física de São Carlos - USP (2000).

[33] Abramowitz, M.; Stegun; I. A., Handbook of mathematical functions: with fomulas, graphs and mathematical tables, Nova Iorque, Dover (1964).

[34] Fetter, A.L.; Walecka, J.D.. Quantum Theory of Many-Particle Systems, Nova Iorque, McGrawHill (1971). 